Cite this: Nat. Prod. Rep., 2013, 30, 108

\section{Ribosomally synthesized and post-translationally modified peptide natural products: overview and recommendations for a universal nomenclature}

\author{
Paul G. Arnison, ${ }^{a}$ Mervyn J. Bibb, ${ }^{b}$ Gabriele Bierbaum, ${ }^{c}$ Albert A. Bowers, ${ }^{d}$

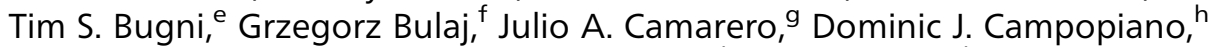 \\ Gregory L. Challis, ${ }^{i}$ Jon Clardy, ${ }^{j}$ Paul D. Cotter, ${ }^{k}$ David J. Craik, ${ }^{\prime}$ Michael Dawson, ${ }^{\mathrm{m}}$ \\ Elke Dittmann, ${ }^{\mathrm{n}}$ Stefano Donadio, ${ }^{\circ}$ Pieter C. Dorrestein, ${ }^{\mathrm{p}}$ Karl-Dieter Entian, ${ }^{\mathrm{q}}$ \\ Michael A. Fischbach, ${ }^{r}$ John S. Garavelli, ${ }^{\text {s }}$ Ulf Göransson, ${ }^{t}$ Christian W. Gruber, ${ }^{u}$ \\ Daniel H. Haft, ${ }^{v}$ Thomas K. Hemscheidt, ${ }^{\text {}}$ Christian Hertweck, ${ }^{x}$ Colin Hill, ${ }^{y}$ \\ Alexander R. Horswill, ${ }^{z}$ Marcel Jaspars, ${ }^{a}$ Wendy L. Kelly, ${ }^{\text {ab }}$ Judith P. Klinman, ${ }^{\text {ac }}$ \\ Oscar P. Kuipers, ${ }^{\text {ad }}$ A. James Link, ${ }^{\text {ae }}$ Wen Liu, ${ }^{\text {af }}$ Mohamed A. Marahiel, ${ }^{\text {ag }}$ \\ Douglas A. Mitchell, ${ }^{\text {h }}$ Gert N. Moll, ${ }^{\text {ai }}$ Bradley S. Moore, ${ }^{\text {aj }}$ Rolf Müller, ${ }^{\text {ak }}$ Satish K. Nair, ${ }^{\text {al }}$ \\ Ingolf F. Nes, ${ }^{\text {am }}$ Gillian E. Norris, ${ }^{\text {an }}$ Baldomero M. Olivera, ${ }^{\text {ao }}$ Hiroyasu Onaka, ${ }^{\text {ap }}$

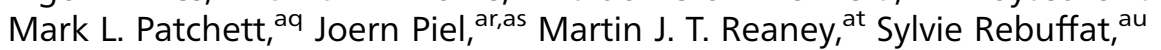 \\ R. Paul Ross, ${ }^{\text {av }}$ Hans-Georg Sahl, ${ }^{\text {aw }}$ Eric W. Schmidt, ${ }^{\text {ax }}$ Michael E. Selsted, ${ }^{\text {ay }}$ \\ Konstantin Severinov, ${ }^{\text {az }}$ Ben Shen, ${ }^{\text {ba }}$ Kaarina Sivonen, ${ }^{\text {b }}$ Leif Smith, $^{\text {bc }}$ Torsten Stein, ${ }^{\text {bd }}$ \\ Roderich D. Süssmuth, ${ }^{\text {be } J o h n ~ R . ~ T a g g, ~}{ }^{\text {bf }}$ Gong-Li Tang, ${ }^{\text {bg }}$ Andrew W. Truman, ${ }^{\text {bh }}$ \\ John C. Vederas, ${ }^{\text {bi }}$ Christopher T. Walsh, ${ }^{\text {bj }}$ Jonathan D. Walton, ${ }^{\text {bk }}$ Silke C. Wenzel, ${ }^{\text {bl }}$ \\ Joanne M. Willey ${ }^{\mathrm{bm}}$ and Wilfred A. van der Donk*bn
}

Covering: 1988 to 2012

Received 8th August 2012

This review presents recommended nomenclature for the biosynthesis of ribosomally synthesized and posttranslationally modified peptides (RiPPs), a rapidly growing class of natural products. The current knowledge regarding the biosynthesis of the $>20$ distinct compound classes is also reviewed, and commonalities are discussed.

www.rsc.org/npr
6.2 Specific recommendations for the cyanobactin family

7 Thiopeptides

7.1 Structural classifications of thiopeptides

7.2 Biological activities of thiopeptides

7.3 Biosynthesis of thiopeptides

7.4 Specific recommendations for the thiopeptide family

8 Bottromycins

8.1 Biosynthesis of the bottromycins

8.2 Specific recommendations for the bottromycin family

9 Microcins

9.1 Biological activities of microcins

9.2 Microcin biosynthesis

9.3 Specific recommendations for the microcin family

10 Lasso peptides

10.1 Biological activities of lasso peptides

10.2 Lasso peptide biosynthesis

10.3 Specific recommendations for the lasso peptide family

11 Microviridins 
11.1 Biological activities of microviridins

11.2 Biosynthesis of microviridins

11.3 Specific recommendations for the microviridin family

12 Sactipeptides: peptides with cysteine sulfur to $\alpha$-carbon crosslinks

12.1 Specific recommendations for sulfur to $\alpha$-carbon linked peptides

13 Bacterial head-to-tail cyclized peptides

13.1 Biosynthesis of bacterial head-to-tail cyclized peptides

13.2 Specific recommendations for bacterial $\mathrm{N}$-to-C cyclized peptides

14 Amatoxins and phallotoxins

15 Cyclotides

15.1 Biological activities of cyclotides

15.2 Cyclotide biosynthesis

15.3 Specific recommendations for the cyclotide family

16 Orbitides: plant cyclic peptides lacking disulfide bonds

16.1 Perspective

16.2 Biosynthesis of orbitides

16.3 Biosynthesis of orbitides in Linaceae

16.4 Biosynthesis of orbitides in Euphorbiaceae

16.5 Relationship to other RiPP subfamilies

16.6 Biological activity

16.7 Specific recommendations for the orbitide family

17 Conopeptides and other toxoglossan venom peptides

17.1 Biological and toxicological perspectives

17.2 Conopeptide structures and biological activities

17.3 The conantokin superfamily and $\gamma$-carboxylation of glutamate

17.4 Biosynthesis of conopeptides

18 Glycocins

18.1 Recommendations for the glycocin family

19 Autoinducing peptides, ComX, methanobactin, and $\mathrm{N}$ formylated peptides

20 PQQ, pantocin, and thyroid hormones

21 Shorthand linear notations for RiPPs

22 Outlook

23 Acknowledgements

24 References

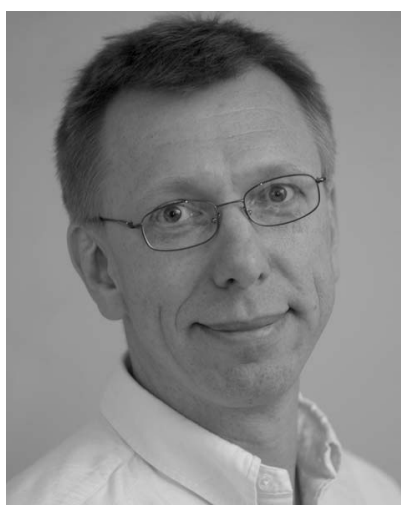

Wilfred van der Donk received a M.S. from Leiden University, the Netherlands, with Prof. Jan Reedijk, and a Ph.D. in organic chemistry with Kevin Burgess at Rice University. He was a Jane Coffin Childs postdoctoral fellow with JoAnne Stubbe at MIT, and joined the faculty at the University of Illinois at Urbana-Champaign in 1997, where he holds the Richard E. Heckert Chair in Chemistry. Since 2008, he is an Investigator of the Howard Hughes Medical Institute. His laboratory aims to understand the molecular mechanisms of enzyme catalysis. Of particular interests are enzymatic reactions in the biosynthesis of natural products, including several classes of RiPPs.
${ }^{a}$ Prairie Plant Systems Inc, Botanical Alternatives Inc, Suite 176, 8B-3110 8th Street E, Saskatoon, SK, S7H OW2, Canada

${ }^{b}$ Department of Molecular Microbiology, John Innes Centre, Norwich Research Park, Norwich NR4 7UH, UK

'Institute of Medical Microbiology, Immunology and Parasitology, University of Bonn, Bonn, Germany

${ }^{a}$ Division of Chemical Biology and Medicinal Chemistry, UNC Eshelman School of Pharmacy University of North Carolina, Chapel Hill, NC 27599, USA

${ }^{e}$ Pharmaceutical Sciences Division, University of Wisconsin-Madison, Wisconsin, 53705, USA

${ }^{f}$ Department of Medicinal Chemistry, University of Utah, Salt Lake City, UT, USA

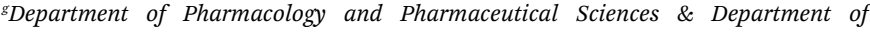
Chemistry, University of Southern California, 1985 Zonal Avenue, Los Angeles, CA 90033, USA

${ }^{h}$ School of Chemistry, EaStCHEM, University of Edinburgh, West Mains Road, Edinburgh, EH9 3JJ, UK

${ }^{i}$ Department of Chemistry, University of Warwick, Coventry, CV4 7AL, United Kingdom ${ }^{i}$ Department of Biological Chemistry and Molecular Pharmacology, Harvard Medical School, 240 Longwood Avenue, Boston, MA 02115, USA

${ }^{k}$ Teagasc Food Research Centre, Moorepark, Fermoy, Co. and Alimentary Pharmabiotic Centre, Cork, Ireland

IInstitute for Molecular Bioscience, The University of Queensland, Brisbane, Queensland 4072, Australia

${ }^{m}$ Novacta Biosystems Ltd, UH Innovation Centre, College Lane, Hatfield, Herts AL1O 9AB, United Kingdom

${ }^{n}$ University of Potsdam, Institute for Biochemistry and Biology, Karl-Liebknecht-Str. 24/25, 14476 Potsdam-Golm, Germany

${ }^{\circ}$ NAICONS Scrl, Via G. Fantoli 16/15, 20138 Milan, Italy

${ }^{p}$ Department of Chemistry and Biochemistry, University of California at San Diego, La Jolla, California 92093, USA

IInstitute Molecular Biosciences, Excellence Cluster: Macromolecular Complexes, Goethe University Frankfurt, Max-von-Laue Str. 9, 60438 Frankfurt/M., Germany

${ }^{r}$ Department of Bioengineering and Therapeutic Sciences, California Institute for Quantitative Biosciences, University of California, San Francisco, California 94158, USA

${ }^{5}$ Center for Bioinformatics \& Computational Biology, Delaware Biotechnology Institute, University of Delaware, 15 Innovation Way, Suite 205, Newark, DE 19711-5449, USA ${ }^{t}$ Division of Pharmacognosy, Dept Medicinal Chemistry, Uppsala University, BMC Box 574, SE 75123 Uppsala, Sweden

${ }^{u}$ Medical University of Vienna, Center for Physiology and Pharmacology, Schwarzspanierstr.17, A-1090 Vienna, Austria

vJ. Craig Venter Institute, 9704 Medical Center Drive, Rockville, Maryland 20850, USA "Department of Chemistry, University of Hawai'i at Manoa, 2545 McCarthy Mall, Honolulu, HI 96822-2275, USA

${ }^{\times}$Leibniz Institute for Natural Product Research and Infection Biology (HKI), Beutenbergstr. 11a, 07745 Jena, Germany

${ }^{y}$ Department of Microbiology and Alimentary Pharmabiotic Centre, University College Cork, Ireland

"Department of Microbiology, Carver College of Medicine, University of Iowa, Iowa City, IA 52242, USA

${ }^{a a}$ Department of Chemistry, University of Aberdeen, Meston Walk, Aberdeen AB24 3UE, ScotlandUK

${ }^{a b}$ School of Chemistry and Biochemistry and the Parker H. Petit Institute for Bioengineering and Bioscience, Georgia Institute of Technology, Atlanta, Georgia 30332, USA

${ }^{a c}$ Department of Chemistry, Department of Molecular and Cell Biology, University of California, Berkeley, CA 94720, USA

${ }^{a d}$ Molecular Genetics, University of Groningen, Nijenborgh 7, 9747 AG, Groningen, The Netherlands

${ }^{a e}$ Department of Chemical and Biological Engineering, A207 Engineering Quadrangle, Princeton University, Princeton, New Jersey 08544, USA

${ }^{a f S t a t e}$ Key Laboratory of Bioorganic and Natural Products Chemistry, Shanghai Institute of Organic Chemistry, Chinese Academy of Sciences, Shanghai 200032, China ${ }^{a s}$ Fachbereich Chemie/Biochemie der Philipps Universität Marburg, Hans-Meerwein-Str., D-35032 Marburg, Germany 
${ }^{a h}$ Departments of Chemistry and Microbiology and the Institute for Genomic Biology, University of Illinois at Urbana-Champaign, Urbana, Illinois 61801, USA

${ }^{a}$ Lanthiopharma, Nijenborgh 4, 9747 AG Groningen, The Netherlands

${ }^{a j}$ Center for Marine Biotechnology and Biomedicine, Scripps Institution of Oceanography, University of California at San Diego, 9500 Gilman Drive, La Jolla, CA 92093, USA

${ }^{a k}$ Helmholtz-Institute for Pharmaceutical Research Saarland (HIPS), Helmholtz Center for Infectious Research (HZI), Department Microbial Natural Products, Saarland University, Campus C2 3, 66123 Saarbrücken, Germany

${ }^{a l}$ Department of Biochemistry, Center for Biophysics and Computational Biology, University of Illinois, 600 S. Mathews Ave, Urbana, IL 61801, USA

${ }^{a m}$ Laboratory of Microbial Gene Technology Department of Chemistry, Biotechnology and Food Science, Norwegian University of Life Sciences, Biotechnology Building, N1432 Aas, Norway

${ }^{a n}$ Institute of Molecular BioSciences, Massey University, Private Bag 11222, Palmerston North, New Zealand

${ }^{a o}$ Department of Biology, University of Utah, Salt Lake City, Utah 84112, USA

${ }^{a p}$ Biotechnology Research Center, Toyama Prefectural University, Toyama Japan

${ }^{a q}$ Institute of Molecular BioSciences, Massey University, Private Bag 11222, Palmerston North, New Zealand

arKekule Institute of Organic Chemistry and Biochemistry, University of Bonn, Gerhard-Domagk-Str. 1, 53121 Bonn, Germany

as Institute of Microbiology, Eidgenössische Technische Hochschule (ETH) Zurich, Wolfgang-Pauli-Str. 10, 8093 Zurich, Switzerland

${ }^{a t}$ Department of Plant Sciences, University of Saskatchewan, Saskatoon, SK, Canada, $S 7 N 5 A 8$

${ }^{a u}$ Laboratory of Communication Molecules and Adaptation of Microorganisms, Muséum National d'Histoire Naturelle, Paris, France

${ }^{a v}$ Teagasc Food Research Centre, Moorepark, Fermoy, Co., and Alimentary Pharmabiotic Centre, Cork, Ireland

${ }^{a w}$ University of Bonn, Institute for Medical Microbiology, Immunology and Parasitology, Pharmaceutical Microbiology Section, Meckenheimer Allee 168, D-53115 Bonn, Germany

${ }^{a x}$ Department of Medicinal Chemistry and Department of Biology, University of Utah, Salt Lake City, Utah 84112, USA

${ }^{a y}$ Department of Pathology, Keck School of Medicine of USC, 2011 Zonal Ave., HMR 204, Los Angeles, California 90033, USA

${ }^{a z}$ Waksman Institute, Department of Genetics, Rutgers University, Piscataway, New Jersey 08854, USA

${ }^{b a}$ Departments of Chemistry and Molecular Therapeutics, The Natural Products Library Initiative at the Scripps Research Institute, The Scripps Research Institute, 130 Scripps Way, \#3A1, Jupiter, Florida 33458, USA

${ }^{b b}$ Department of Applied Chemistry and Microbiology, University of Helsinki, P.O. Box 56, Viikki Biocenter, o0014 Helsinki, Finland

${ }^{b c}$ Department of Biological Sciences, Texas A\&M University, College Station, TX 77843, USA

${ }^{b d}$ Institute of Molecular Biotechnology, Department of Chemistry, University of Aalen, 73430 Aalen, Germany

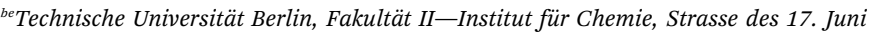
124, 10623 Berlin, Germany

${ }^{b f}$ BLIS Technologies Limited Level 1, Centre for Innovation, 87 St David Street, PO Box 56, Dunedin 9054, New Zealand

${ }^{b 5}$ State Key Laboratory of Bio-organic and Natural Products Chemistry, Shanghai Institute of Organic Chemistry, Chinese Academy of Sciences, 345 Lingling Road, Shanghai 200032, China

${ }^{b h}$ Department of Chemistry, University of Cambridge, Cambridge, United Kingdom, CB2 1EW

${ }^{b i}$ Department of Chemistry, University of Alberta, Edmonton, Alberta, Canada, T6G $2 G 2$

${ }^{b j}$ Department of Biological Chemistry \& Molecular Pharmacology, Harvard Medical School, 240 Longwood Avenue, Boston, Massachusetts 02115, USA

${ }^{b k}$ Department of Energy, Plant Research Laboratory, Michigan State University, East Lansing, Michigan 48824, USA

${ }^{b l}$ Helmholtz-Institute for Pharmaceutical Research Saarland (HIPS), Helmholtz Center for Infectious Research (HZI), Department Microbial Natural Products, Saarland University, Campus C2 3, 66123 Saarbrücken, Germany

\section{Introduction}

Natural products have played key roles over the past century in advancing our understanding of biology and in the development of medicine. Research in the 20th century identified many classes of natural products with four groups being particularly prevalent: terpenoids, alkaloids, polyketides, and non-ribosomal peptides. The genome sequencing efforts of the first decade of the 21st century have revealed that another major class is formed by ribosomally synthesized and post-translationally modified peptides. These molecules are produced in all three domains of life, their biosynthetic genes are ubiquitous in the currently sequenced genomes ${ }^{1-8}$ and transcriptomes, ${ }^{9,10}$ and their structural diversity is vast. ${ }^{11}$ The extensive post-translational/co-translational modifications endow these peptides with structures not directly accessible for natural ribosomal peptides, typically restricting conformational flexibility to allow better target recognition, to increase metabolic and chemical stability, and to augment chemical functionality.

Because the common features of their biosynthetic pathways have only recently been recognized, at present the nomenclature used in different communities investigating subgroups of natural products of ribosomal origin is non-uniform, confusing, and in some cases even contradictory. In order to define a uniform nomenclature, the authors of this review engaged in a discussion over the summer and fall of 2011, and the consensus recommendations of these discussions are presented herein. In addition, this review provides an overview of the structures of and the biosynthetic processes leading to this large group of natural products. Select examples are covered from bacteria, fungi, plants, and cone snails. Post-translational in the context of this review is defined as any peptide chain modification occurring after the initiation of translation.

Historically, ribosomally synthesized and post-translationally modified peptides have been subdivided based on either the producing organisms (e.g. microcins produced by Gramnegative bacteria) $)^{12}$ or their biological activities (e.g. bacteriocins). ${ }^{13}$ Unlike the designations of the other four major classes of natural products listed above, an overarching designation for these peptide natural products based on structural and biosynthetic commonality is currently lacking. We propose that the biosynthetic pathway to these compounds be referred to as Post-Ribosomal Peptide Synthesis (PRPS), in line with the currently used designation of Non-Ribosomal Peptide Synthetase (NRPS) enzymes that catalyze modular assembly line biosynthesis of peptide natural products. ${ }^{14,15}$ Furthermore, we propose to designate the resulting ribosomally-synthesized and post-translationally-modified peptides as RiPPs, irrespective of their biological functions. The name "Post-Ribosomal Peptides" was also considered as a logical extension of NRPs, but because this designation would not capture the critical post-translational modifications, the name RiPPs was ultimately

\footnotetext{
${ }^{b m}$ Department of Biology and Science Education, Hofstra University-North Shore/LIJ, School of Medicine, Hempstead, New York 11549, USA

${ }^{b n}$ Department of Chemistry and Howard Hughes Medical Institute, University of Illinois at Urbana-Champaign, 600 S. Mathews Avenue, Urbana, Illinois 61801, USA
} 
Table 1 Proposed nomenclature for RiPP biosynthesis

\begin{tabular}{|c|c|c|c|}
\hline Nomenclature proposed herein & Previous designations & & \\
\hline Precursor peptide & $\begin{array}{l}\text { Prepeptide (lantibiotics, } \\
\text { microviridins) }\end{array}$ & $\begin{array}{l}\text { Prepropeptide (lantibiotics, cyclic } \\
\text { bacteriocins) }\end{array}$ & $\begin{array}{l}\text { Structural peptide (translation } \\
\text { product of the structural gene; } \\
\text { lantibiotics, thiopeptides) }\end{array}$ \\
\hline Leader peptide & $\begin{array}{l}\text { Propeptide (microcins, } \\
\text { conopeptides, cyclotides) }\end{array}$ & Pro-region (conopeptides) & Intervening region (conopeptides) \\
\hline Core peptide & $\begin{array}{l}\text { Propeptide (lantibiotics, } \\
\text { mycotoxins, cytolysins, cyclic } \\
\text { bacteriocins) }\end{array}$ & $\begin{array}{l}\text { Structural peptide (lantibiotics, } \\
\text { thiopeptides, cyanobactins) }\end{array}$ & Toxin region (conopeptides) \\
\hline Recognition sequences & NTR, N-terminal repeats (cyclotides) & & \\
\hline
\end{tabular}

preferred. Similarly, the name "Ribosomal Natural Products" was considered but the resulting acronym, RNPs, was deemed too close to NRP, the acronym for non-ribosomal peptides. Finally "Ribosomal Peptides" was discussed, but since confusion could arise with peptides that make up the ribosome, RiPPs was again preferred. A size limit of $10 \mathrm{kDa}$ is imposed on RiPPs to exclude post-translationally modified proteins. We note that a small number of post-translationally modified natural metabolites are not well described by this nomenclature because the final products lack any peptide bonds, such as the cofactor pyrroloquinoline quinone (PQQ), and the thyroid hormones (see section 20).

\subsection{Common features of RiPP biosynthesis}

Nearly all compounds produced by PRPS are initially synthesized as a longer precursor peptide, typically $\sim 20-110$ residues in length, encoded by a structural gene. In the past, the various segments of this precursor peptide have been given different, sometimes conflicting names for different RiPP subclasses (Table 1). We propose here a uniform naming scheme in which the segment of the precursor peptide that will be transformed into the natural product is called the core peptide or core region

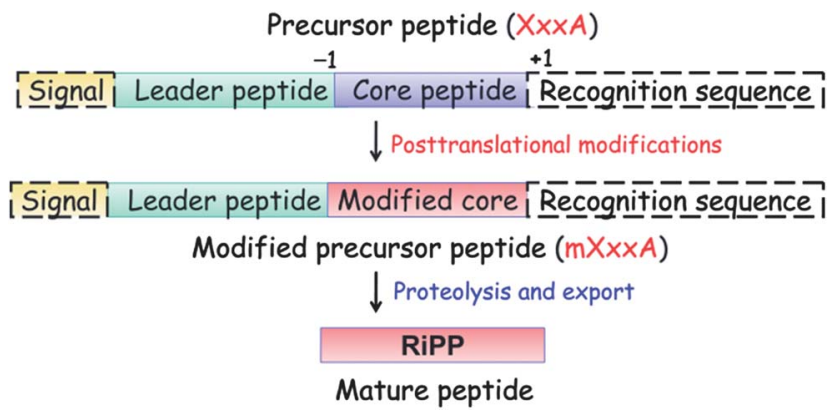

Fig. 1 General biosynthetic pathway for RiPPs. The precursor peptide contains a core region that is transformed into the mature product. Many of the posttranslational modifications are guided by leader peptide and the recognition sequences as discussed in this review for subclasses of RiPPs. Products of eukaryotic origin also often contain an $\mathrm{N}$-terminal signal peptide that directs the peptide to specialized compartments for modification and secretion. C-terminal recognition sequences are sometimes also present for peptide cyclization (e.g. see sections on cyanobactins, amatoxins, cyclotides, and orbitides). In the case of the bottromycins, a leader-like peptide is appended at the C-terminus of the core peptide. ${ }^{17-20}$
(Fig. 1). A distinction can be made with respect to the unmodified core peptide (UCP) in the precursor peptide and the modified core peptide (MCP) after the post-translational modifications. In most RiPPs, a leader peptide or leader sequence is appended to the $\mathrm{N}$-terminus of the core peptide that is usually important for recognition by many of the post-translational modification enzymes and for export. ${ }^{16}$ In some more rare examples such as the bottromycins (section 8), ${ }^{17-20}$ a leader peptide is not attached at the N-terminus, but rather at the $\mathrm{C}$ terminus of the core peptide and has been termed a "follower" peptide. For eukaryotic peptides such as the cyclotides and conopeptides discussed in this review, a signal sequence is often found $\mathrm{N}$-terminal to the leader peptide that directs the peptide to the specific cellular compartments where the post-translational modifications will take place. Finally, some peptides have C-terminal recognition sequences that are important for excision and cyclization. ${ }^{21-23}$ The unmodified precursor peptide is generally designated " $\mathrm{A}$ " (encoded by the $x x x A$ gene), but for some classes different designations have been used historically (see discussion of individual RiPP families). The modified precursor peptide prior to proteolytic removal of the leader peptide can be abbreviated as mXxxA (e.g. mLanA; Fig. 1).

It is noteworthy that the leader peptide-guided strategy for the biosynthesis of RiPPs results in highly evolvable pathways because many of the post-translational processing enzymes recognize the leader peptide and are highly permissive with respect to mutations in the core peptide. Indeed, the core regions are naturally hypervariable for subsets of the RiPP classes discussed in this review,,$^{3,22,24-26}$ and engineering studies have further demonstrated the plasticity of the biosynthetic enzymes. ${ }^{27-44}$ The relatively small number of enzymes involved in the maturation pathways also facilitates natural evolution, and the multiplicity of pathways towards the same types of chemical structures illustrates the convergent evolution of efficient, ribosome-based biosynthetic strategies. Collectively, these features highlight the potential evolutionary advantage of accessing high chemical diversity at low genetic cost. We note that these very same attributes also prove advantageous with respect to genome mining strategies as heterologous production of RiPPs is aided by short pathways and is not limited by supply of precursors.

The explosion in sequence information has also revealed that many biosynthetic pathways utilize common enzymes for a subset of the post-translational modifications. For instance, the 
Ser/Thr dehydratases involved in the biosynthesis of lanthi onine-containing peptides (lanthipeptides) are also used in the biosynthetic pathways to the proteusins, ${ }^{45}$ thiopeptides, and some linear azole-containing peptides (LAPs), ${ }^{\mathbf{4 4 6 - 4 9}}$ while some of the enzymes involved in oxazol(in)e and thiazol(in)e formation in thiopeptides ${ }^{46-50}$ are also used for the biosynthesis of LAPs $^{50-52}$ and bottromycins ${ }^{17-20}$ and are also often found in cyanobactin biosynthetic gene clusters. ${ }^{21}$ Similarly, genes encoding radical-SAM dependent methyltransferases are found in the biosynthetic gene clusters of thiopeptides ${ }^{46}$ proteusins,${ }^{45}$ and bottromycins. ${ }^{17-20}$ Thus, acquisition of new post-translational modification enzymes appears to drive evolution to new structures. On the other hand, some post-translational modifications appear to have evolved convergently, such as the different ways to achieve head-to-tail (N-to-C) cyclization in the cyanobactins, ${ }^{53}$ amatoxins, ${ }^{54}$ circular bacteriocins, ${ }^{55}$ and cyclotides, ${ }^{56}$ and the different ways in which thioether crosslinks are formed in various classes of lanthipeptides. ${ }^{57}$ Post-translational modifications involving Cys residues occur especially frequently in RiPPs. Sulfur chemistry converts the thiols of cysteines to disulfides (cyclotides, conopeptides, lanthipeptides, cyanobactins, lasso peptides, sactipeptides, and glycocins), thioethers (lanthipeptides, sactipeptides, phalloidins, some thiopeptides), thiazol(in)es (thiopeptides, LAPs, cyanobactins, bottromycins), and sulfoxides (lanthipeptides, amatoxins). Additional common features are macrocyclization to increase metabolic stability and decrease conformational flexibility, and modifications to the $\mathrm{N}$ - and $\mathrm{C}$-termini to limit the susceptibility to degradation by exoproteases.

Although the details of recognition of leader peptides by the biosynthetic enzymes are still mostly unknown, many leader peptides have a propensity to form $\alpha$-helices, ${ }^{58-64}$ either in solution or when bound to the biosynthetic proteins.65,66 The leader peptides are thought to play multiple roles in post-translational modification, export, and immunity. Furthermore, several pieces of evidence suggest that different biosynthetic enzymes in a pathway recognize different segments of the leader peptides. ${ }^{59,64,67,68}$ Leader peptide removal can take place in one proteolytic step or in multiple proteolytic steps. In regards to the numbering of the residues of the leader peptide, it is recommended to count backwards from the final cleavage site and add a minus sign before the number, e.g. the last residue that is not incorporated in the final RiPP is numbered -1 and counting then commences $-2,-3$ etc towards the N-terminus (Fig. 1). Similarly, C-terminal recognition sequences/follower peptides can be numbered with a plus sign from the site of final cleavage (e.g. the $\mathrm{N}$-terminal residue of the C-terminal recognition sequence would be numbered +1 , Fig. 1) counting up from this site towards the Cterminus. Counting schemes in the case of multiple core peptides and recognition sequences (e.g. in the cyanobactins, cyclotides, and orbitides) are case dependent.

The sections below briefly discuss the salient features of these various compound classes including their defining structural motifs, common biosynthetic pathways, subdivisions into subclasses, and recommendations regarding nomenclature. This review covers most bacterial RiPPs that have been identified as well as select examples from fungi (amatoxins and phallotoxins), plants (cyclotides and orbitides), and metazoans (conopeptides from cone snails). Not covered, but often made by a similar biosynthetic logic if they are post-translationally modified, are defensins ${ }^{69-71}$ and the venom peptides from insects and reptiles. ${ }^{72-75}$

\section{Lanthipeptides}

\subsection{Biosynthesis}

First reported in $1928,{ }^{76}$ nisin is one of the longest known RiPPs, but its structure was not elucidated until 1971 (Fig. 2A). ${ }^{77}$ It contains the meso-lanthionine and 3-methyllanthionine residues that define the lanthipeptide (for lanthionine-containing peptides) class of molecules. Lanthipeptides that have antimicrobial activity are called lantibiotics. ${ }^{78}$ Lanthionine (Lan) consists of two alanine residues crosslinked via a thioether linkage that connects their $\beta$-carbons; 3-methyllanthionine (MeLan) contains one additional methyl group (Fig. 2B). The ribosomal origin of lanthionine-containing peptides was first proposed in $1970^{79}$ and experimentally verified in 1988 when the biosynthetic gene cluster for epidermin was sequenced. ${ }^{78}$ Shortly thereafter, the precursor genes for nisin, ${ }^{\mathbf{8 0}, 81}$ subtilin, ${ }^{82}$ and Pep5 ${ }^{83}$ were identified. The biosynthetic genes have been designated the generic locus symbol lan, with a more specific genotypic designation for each lanthipeptide member (e.g., nis for nisin, ${ }^{81}$ gar for actagardine,$^{84} \mathrm{mrs}$ for mersacidin, ${ }^{85} \operatorname{cin}$ for cinnamycin ${ }^{86}$ ). The Lan and MeLan residues are introduced in a two-step post-translational modification process. In the first step, Ser and Thr residues in the precursor peptide are dehydrated to dehydroalanine (Dha) and dehydrobutyrine (Dhb) residues, respectively, usually via a phosphorylated intermediate (Fig. 2B). ${ }^{87-89}$ The thioether crosslinks are formed subsequently via a Michael-type addition by Cys residues onto the dehydro amino acids.

At present lanthipeptides are classified into four different classes depending on the biosynthetic enzymes that install the Lan and MeLan motifs (Fig. 3). ${ }^{57}$ For class I lanthipeptides, the dehydration is carried out by a dedicated dehydratase generically called LanB, ${ }^{\mathbf{9 0 - 9 3}}$ with a more specific designation for each lanthipeptide (e.g. NisB for nisin). Cyclization of class I lanthipeptides is catalyzed by a LanC cyclase. ${ }^{\mathbf{9 4 - 9 6}}$ Several pieces of evidence suggest these proteins form a multienzyme complex. ${ }^{97}$ For class II, ${ }^{88,99}$ III, ${ }^{88}$ and IV $^{89}$ lanthipeptides, dehydration and cyclization is carried out by bifunctional lanthionine synthetases. The class II LanM lanthionine synthetases have $\mathrm{N}$ terminal dehydration domains that do not display sequence homology with other enzymes in the databases. However, the Cterminal cyclization domains have homology with the LanC cyclases of class I (Fig. 3). For both class III and IV lanthipeptides, dehydration is carried out by successive actions of a central kinase domain and an N-terminal phosphoSer/phosphoThr lyase domain, ${ }^{\mathbf{8 9 , 1 0 0}}$ but the class III and IV synthetases differ in their C-terminal cyclization domains (Fig. 3). ${ }^{\mathbf{1 0 1}}$ Although these domains show sequence homology to each other as well as to the LanC proteins, three metal binding residues ${ }^{95,102}$ that are fully conserved in class I, II, and IV cyclases are absent in the class III cyclization domains. ${ }^{\mathbf{1 0 1}}$ A subset of class III enzymes generates an additional 
A

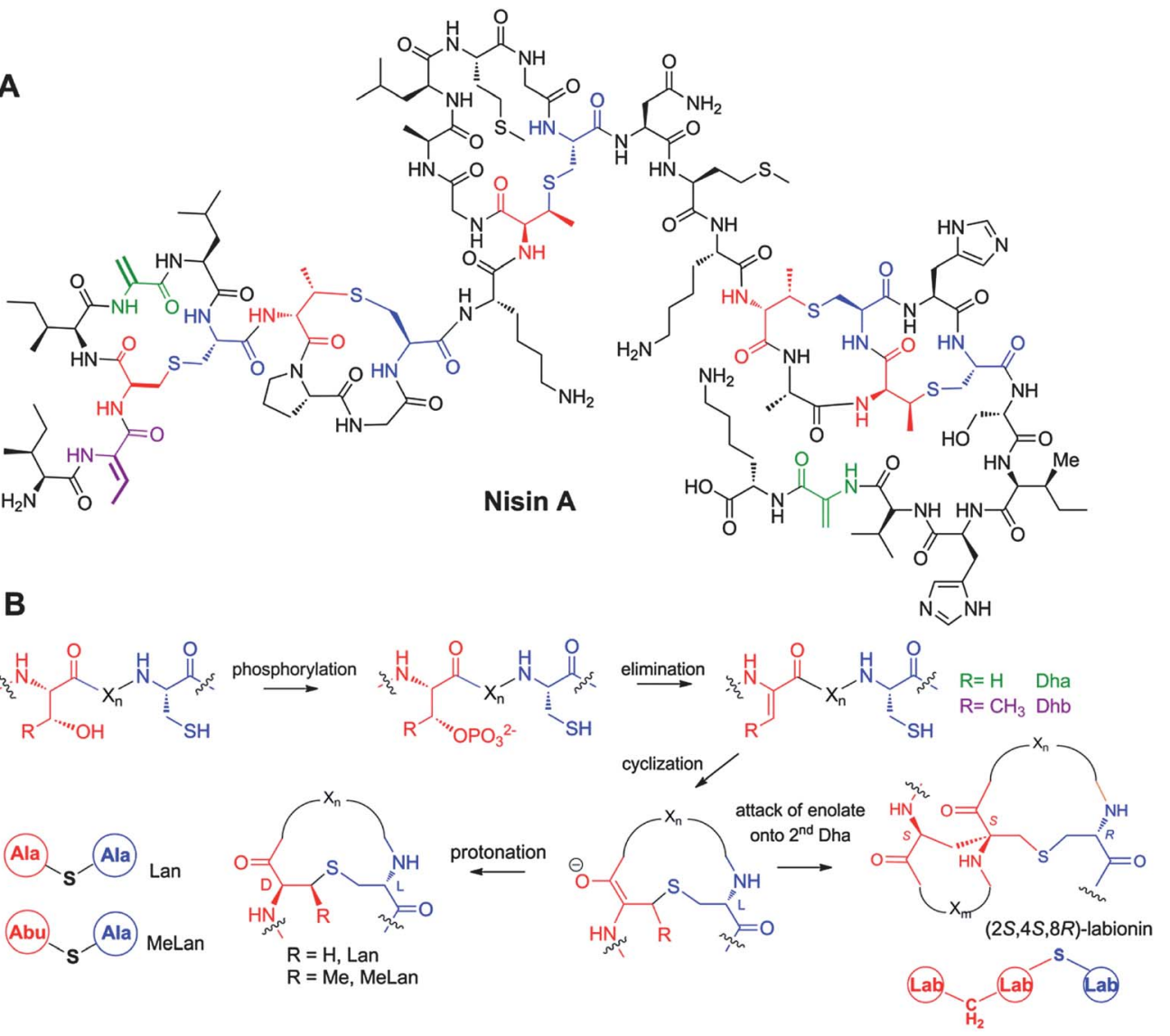

Fig. 2 A. Structure of nisin A. Dha residues are shown in green, Dhb residues in purple. Segments of Lan and MeLan originating from Ser/Thr are depicted in red and segments of (Me)Lan originating from Cys in blue. B. Biosynthetic pathway to (Me)Lan and labionin formation. Ser and Thr residues are first phosphorylated and the phosphate is subsequently eliminated to generate Dha and Dhb residues, respectively. Phosphorylation prior to elimination has been experimentally confirmed for class II-IV lanthipeptides and is postulated for class I lanthipeptides. Subsequent intramolecular conjugate addition by the side chain of Cys generates an enolate. Protonation of the enolate produces the (Me)Lan structures whereas attack of the enolate onto another Dha generates the labionin crosslinks.

carbon-carbon crosslink, putatively by attack of the initially generated enolate onto a second dehydroalanine (Fig. 2B). The structure thus formed is called a labionin (Lab), first detected in the labyrinthopeptins. ${ }^{\mathbf{1 0 3}}$

Lanthipeptide biosynthetic enzymes have demonstrated low substrate specificity, allowing substitutions of those residues in

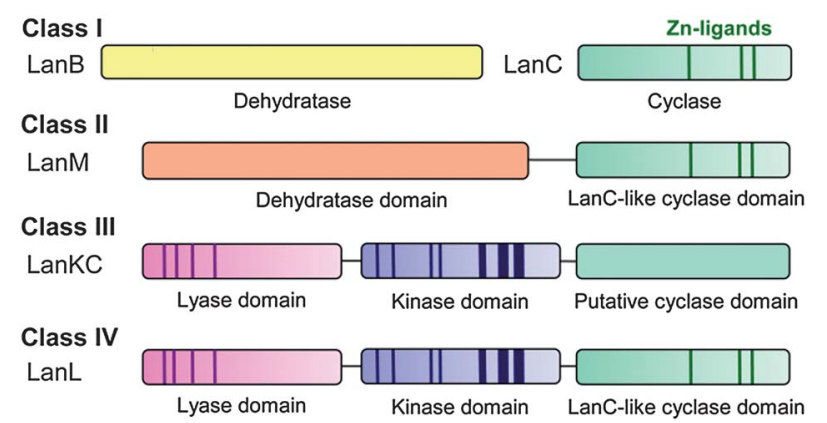

Fig. 3 Four different classes of lanthipeptides are defined by the four different types of biosynthetic enzymes that install the characteristic thioether crosslinks. The dark areas show conserved regions that are important for catalytic activity. The cyclase domain of class III enzymes ${ }^{104}$ has homology with the other cyclase domains/enzymes but lacks the three zinc ligands. the core peptide that are not post-translationally modified with both proteinogenic $\mathrm{c}^{27-30,33-35,105-109}$ and non-proteinogenic amino acids. ${ }^{34,110,111}$ The enzymes that generate the characteristic crosslinks are dependent on the presence of the leader peptide, ${ }^{59,63,78,93,95,99,112-116}$ but several of the enzymes that install other post-translational modifications do not require the leader peptide. ${ }^{117-119}$ Furthermore, non-lanthipeptide sequences attached to LanA leader peptides have been modified by the lanthipeptide biosynthetic machinery..$^{\mathbf{9 2 , 1 2 0 - 1 2 3}}$ For the class II lantibiotic lacticin $481^{116,124}$ and the class I lantibiotic nisin, ${ }^{125}$ it has been demonstrated that the leader peptide does not need to be attached to the core peptide. The results of these studies better fit a model in which leader peptide binding results in a shift in the equilibrium between an inactive and active form of the synthetase towards the latter, rather than models in which the leader peptide pulls the core peptide through the active site or in which leader peptide binding induces a conformational change..$^{59,116}$

\subsection{Structure and biological activities of lanthipeptides}

Recent years have revealed that lanthionine-containing peptides can have functions beyond antimicrobial activities, ${ }^{\mathbf{1 0 1 , 1 0 4 , 1 2 6}}$ and therefore the name lantipeptide was initially 
introduced for all lanthionine and methyllanthionine containing peptides ${ }^{89}$ with lantibiotics forming a large subgroup. As described above, we revise the term lantipeptide here to lanthipeptide as a more faithful representation for lanthioninecontaining peptides regardless of their biological activities.

Nisin (class I) is the best studied lantibiotic and has been used in the food industry to combat food-borne pathogens for more than 40 years. ${ }^{127}$ It binds to lipid II, an essential intermediate in peptidoglycan biosynthesis, resulting in inhibition of cell wall biosynthesis and pore formation. ${ }^{128,129}$ Other notable lanthipeptides include the class II lantibiotic actagardine, a semi-synthetic derivative of which is in development against Clostridium difficile ${ }^{84}$ the class II lantibiotic duramycin that binds phosphatidylethanolamine and is being evaluated for treatment of cystic fibrosis, ${ }^{130}$ and labyrinthopeptin, a class III compound with antiallodynic activity (Fig. 4). ${ }^{103}$ An interesting group of class II lantibiotics are the two-component peptides such as lacticin 3147 and haloduracin. ${ }^{131-134}$ These peptides

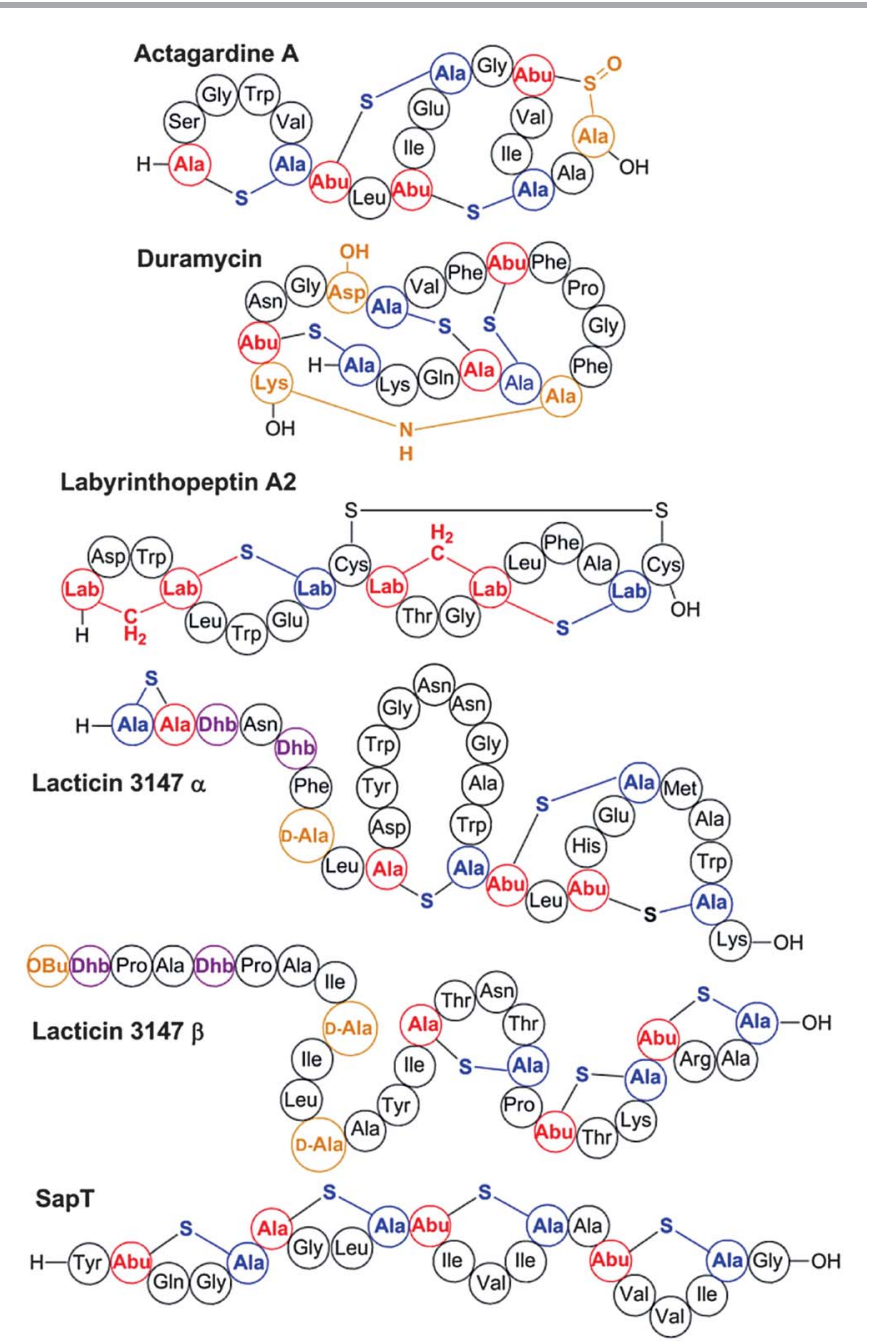

Fig. 4 Some representative lanthipeptides. The same shorthand notation is used as in Fig. 2. Segments of crosslinks originating from Cys are in blue, segments originating from Ser/Thr are in red. Additional post-translational modifications are shown in orange. ${ }^{138} \mathrm{OBu}$, 2-oxobutyric acid; $\mathrm{Asp}-\mathrm{OH}$, (3R)-hydroxy-aspartate; Lys-NH-Ala, lysinoalanine. synergistically kill bacteria with the $\alpha$-peptide binding to lipid II. ${ }^{135,136}$ The $\beta$-peptide is then believed to bind to the complex formed between the $\alpha$-peptide and lipid II and induce pore formation. ${ }^{137}$

The first lanthipeptides shown to have functions other than antimicrobial agents were the morphogenetic peptides SapB and SapT from streptomycetes. ${ }^{101,126}$ These peptides are believed to function as biosurfactants during the formation of aerial hyphae. ${ }^{139}$ Lanthipeptides are defined by the presence of the characteristic (Me)Lan residues, but as many as 15 other posttranslational modifications have been documented, ${ }^{57,128,138}$ with additional modifications likely to be uncovered in the future. Unlike (Me)Lan, Dha, and Dhb formation, these additional modifications appear not to rely on the presence of a leader peptide for the examples investigated thus far. ${ }^{117-119,140}$

Genome database mining has illustrated that the biosynthetic genes for lanthipeptides are distributed much more widely than the Firmicutes and Actinobacteria to which biosynthesis of these compounds was believed to be restricted. Their genes are now also found in certain bacteroidetes, chlamydiae, proteobacteria, and cyanobacteria. ${ }^{26,89,141,142}$ In all, over 90 lanthipeptides are known with hundreds more identified in genomes. . $^{2,36,141-143}$

\subsection{Specific recommendations for the lanthipeptide family}

The name lanthipeptides describes all lanthionine- and methyllanthionine-containing peptides made by PRPS. Lantibiotics form a large subgroup with antimicrobial activities. The gene designations for the various post-translational modification enzymes involved in lanthipeptide biosynthesis are well established and we recommend continued use of the generic name LanA for the precursor peptide, LanBC for the dehydratases and cyclases of class I lanthipeptides, and LanM and LanL for the bifunctional enzymes involved in the biosynthesis of class II and IV compounds, respectively. The class III bifunctional enzymes should be called LanKC irrespective of whether they result in formation of labionines or lanthionines because at present the final products cannot be predicted from the sequences of the synthetases. ${ }^{104,144}$ The suffix -peptin is recommended for naming new members of class III peptides. ${ }^{104,144}$

Further recommendations are the continued use of LanP for Ser proteases that remove part of or the entire leader peptide, LanT for transporters that secrete the lanthipeptides (with or without an N-terminal Cys protease domain), LanEFG for the transporters that are involved in self-resistance, LanI for additional immunity proteins, and LanKR for two-component response regulators. With respect to the enzymes that install the less common PTMs, LanD should be used for the enzymes that oxidatively decarboxylate C-terminal Cys residues, ${ }^{145}$ LanO for various oxidation enzymes, ${ }^{84,140,146}$ LanX for hydroxylases, ${ }^{118,147}$ and LanJ for enzymes that convert Dha to D-Ala. ${ }^{148}$ This recommendation leaves only a small series of letters remaining (lanHNQSWYZ), some of which have already been used to name individual, non-common genes in lanthipeptide gene clusters. ${ }^{149}$ Hence, we recommend the use of a five-letter designation, such as that used for lanKC, for genes that may be 
uncovered in future studies and that have no sequence homology with currently known lanthipeptide biosynthetic genes.

\section{Linaridins}

Linaridins are a recently discovered family of RiPPs that share the presence of thioether crosslinks with the lanthipeptide family but that are generated by a different biosynthetic pathway. Cypemycin is the founding member of the family and it was considered a lanthipeptide because of the presence of a Cterminal aminovinyl cysteine (Fig. 5). ${ }^{\mathbf{1 5 0}}$ These same structures are found in several lantibiotics such as epidermin and mersacidin and are believed to be generated by Michael-type addition of an oxidatively decarboxylated C-terminal Cys onto a Dha, ${ }^{151}$ with the latter formed by dehydration of Ser. However,

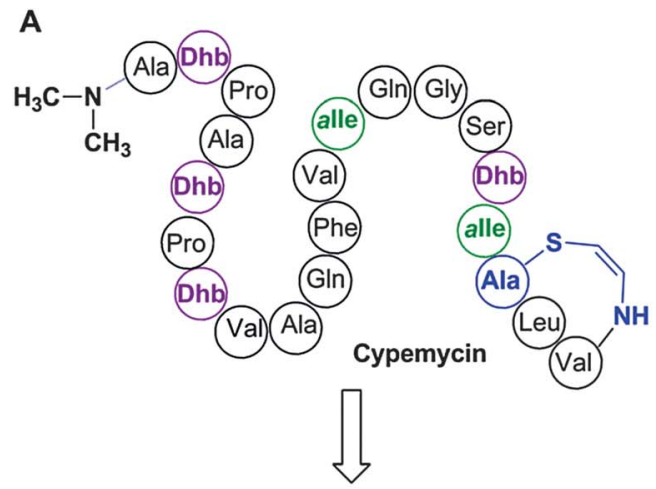

leader peptide- ATPATPTVAQFVIQGSTICLV므

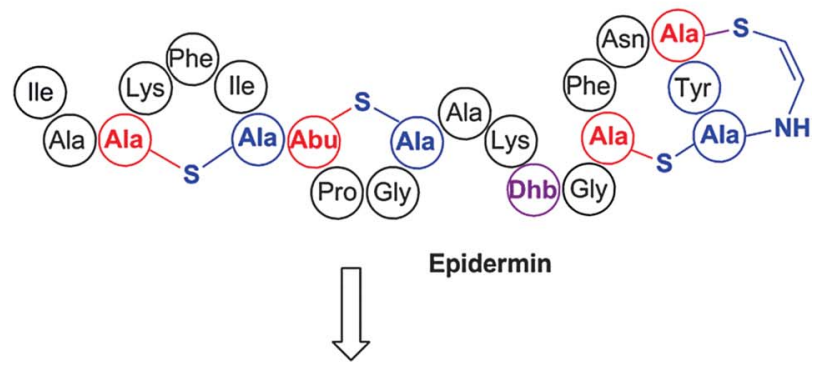

leader peptide- IASKFICTPGCAKTGSFN $\underline{\text { YYC }}$

B

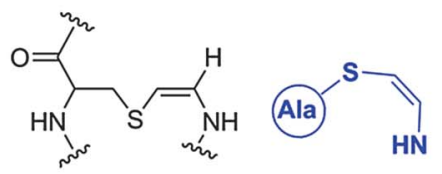

[(Z)-2-aminovinyl]-cysteine (AviCys)

Fig. 5 A. Structure of cypemycin, a representative member of the linaridins. The linear sequence of the precursor peptide is shown below the retrosynthetic arrow to illustrate the biosynthetic origin of each PTM: allo-lle from Ile, Dhb from Thr, and AviCys from two Cys residues (underlined). For comparison, the structure of the lanthipeptide epidermin, which also contains an AviCys structure, is shown but its AviCys is formed from a Ser and Cys residue (underlined). B. Chemical structure of AviCys as well as the shorthand notation used in panel A; the absolute configuration of the AviCys and allo-lle in cypemycin is not known. when the biosynthetic gene cluster of cypemycin was identified, it became clear that none of the four types of lanthipeptide dehydratases were present in the cluster. ${ }^{152}$ Furthermore, the sequence of the core peptide illustrates that the AviCys structure is formed from two Cys residues. Based on these differences from the biosynthetic route to AviCys in lanthipeptides, cypemycin and related peptides found in the genome databases ${ }^{6,152,153}$ have been classified as a separate group, the linaridins. If future research identifies linaridins containing Lan and/or MeLan, it may be that linaridins are better classified as another subclass of the lanthipeptides, but as long as that is not the case, they are grouped separately.

\section{Proteusins}

Polytheonamides (Fig. 6) were reported in 1994 as extremely cytotoxic constituents of a Japanese Theonella swinhoei sponge, ${ }^{154}$ which harbors a large diversity of symbiotic bacteria. ${ }^{155}$ These initial studies and a structural revision in 2005 by the same group ${ }^{\mathbf{1 5 6}}$ established the compounds as highly complex 48-mer peptides containing, in addition to an unprecedented $N$-acyl moiety, an unusually large number of nonproteinogenic residues, including many tert-leucine and other $C$-methylated amino acids. One of the most interesting features is the presence of multiple D-configured units that are localized in near perfect alternation with L-amino acids, reminiscent of the nonribosomally produced linear gramicidins. ${ }^{157}$ Like these compounds, polytheonamides form membrane channels, which is at least in part the basis of their cytotoxicity. ${ }^{158}$ Channel formation occurs by adoption of a $\beta$-helical secondary structure and directional insertion into the membrane aided by the lipophilic $N$-acyl unit. ${ }^{158,159}$ However, unlike the bimolecular gramicidin pores, the much larger polytheonamides are able to span the entire membrane and thus form minimalistic ion channels as single molecules. Because of the highly modified polytheonamide structures it was generally assumed that they are nonribosomal peptides and, as such, the largest known members of this family. Recently, however, metagenomic studies were conducted on the sponge involving screening of a $\sim 1$ million clone DNA library, which revealed that polytheonamides are biosynthesized via a ribosomal pathway. ${ }^{45}$ Such an origin is notable, since it implies the existence of 48 postribosomal modification steps excluding removal of the leader region, making polytheonamides the most extensively modified RiPPs known to date. Particularly noteworthy is the regiospecific epimerization of 18 structurally diverse L-amino acids, which suggests that there is no significant biochemical limitation to the stereo- and regiospecific biosynthesis of configurationally mixed ribosomal products. These structural peculiarities as well as the unusually large leader region of the precursor (see below) classified polytheonamides as members of a new RiPP family, termed proteusins. The metagenomic study also showed that an as-yet uncultivated bacterial symbiont of the sponge is the actual producer, highlighting the potential of environmental bacteria as sources of structurally novel RiPPs. ${ }^{45}$ 


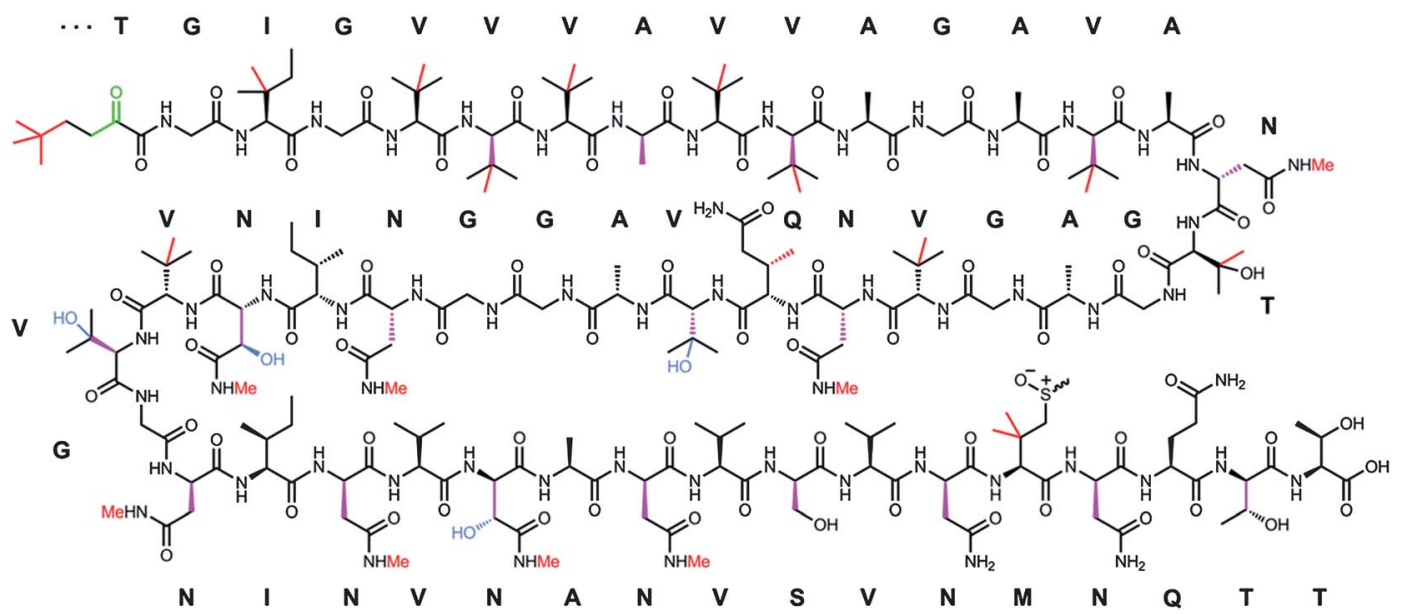

Fig. 6 Structure of polytheonamide A and B and sequence of the precursor core region. The two congeners differ by the configuration of the sulfoxide moiety. Sulfur oxidation is an artifact occurring during isolation. Color code for posttranslations modifications: purple, epimerization; green, dehydration; red, methylation; blue, hydroxylation.

\subsection{Biosynthesis and structural classifications of proteusins}

Biosynthesis of polytheonamides involves a precursor protein (PoyA) with a large leader region that exhibits similarity to nitrile hydratase-like enzymes. By coexpressing the precursor gene with a variety of genes for posttranslational modification, the function of several modifying enzymes was established. ${ }^{45}$ Remarkably, in a first step a single epimerase, PoyD, generates most, and possibly all, D-residues. The enzyme is homologous to members of the radical $S$-adenosyl methionine superfamily ${ }^{\mathbf{1 6 0}}$ and likely acts by abstracting an $\alpha$-hydrogen to form a stabilized amino acyl radical. Hydrogen donation from the backside would then result in epimerization. The source of the second hydrogen is currently unknown. Epimerization occurs unidirectional, which is in contrast to the equilibrium-forming, asyet uncharacterized epimerases associated with, e.g., several modified peptides from animals. ${ }^{161}$ Only few homologues of PoyD are currently listed in GenBank. All contain a characteristic N-terminal domain-like region of unknown function and are associated with nitrile hydratase-like precursors. The second step in polytheonamide biosynthesis is dehydration of a Thr residue that seems to be the biosynthetic source of the unusual $N$-acyl residue (Fig. $7 \mathrm{~A}) .{ }^{45}$ This reaction is performed by
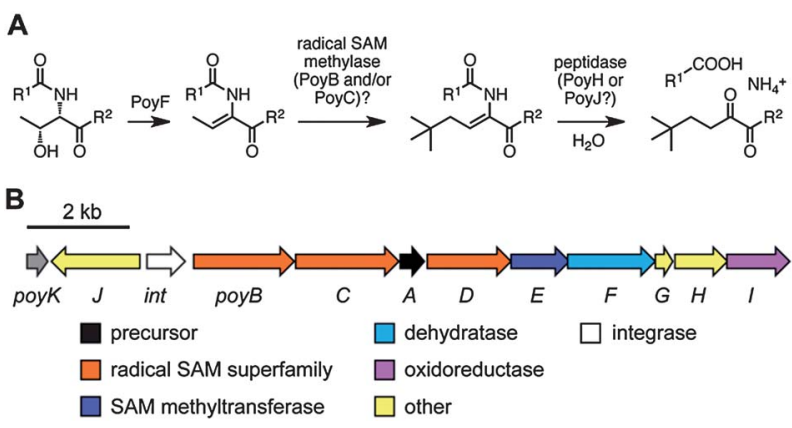

Fig. 7 A. Proposed biosynthesis of the $N$-acyl unit of polytheonamides. The dehydration step was experimentally verified. B. Polytheonamide biosynthetic gene cluster.
PoyF, an enzyme resembling the N-terminal dehydratase domain of LanM-type class II bifunctional lanthionine synthetases (see section 2). Further conversion to the $N$-acyl unit likely proceeds via net $t$-butylation catalyzed by one or more class $\mathrm{B}^{\mathbf{1 6 2}}$ radical-SAM methyltransferases (PoyB and/or $\mathrm{C}$ are candidates), which would add a total of four methyl groups to the methyl group originating from Thr (Fig. 7A). Hydrolytic removal of the leader region would then generate an enamine moiety that would spontaneously form the $\alpha$-keto unit found in polytheonamides, similar to the ketone generation at the $\mathrm{N}$ terminus of Pep5-like lanthipeptides. ${ }^{\mathbf{8 3 , 1 4 0 , 1 4 6 , 1 6 3}}$ Regiospecific generation of the $8 \mathrm{~N}$-methylated Asn residues was shown to be catalyzed by a single $N$-methyltransferase, PoyE. ${ }^{45}$ A general hallmark of the pathway is the extremely streamlined gene cluster that encodes several highly iterative enzymes (Fig. 7B). For all 48 modifications, only 6 enzyme candidates encoded in a $14 \mathrm{~kb}$ cluster were identified. However, it has to be tested whether the remaining as-yet uncharacterized modifications (4 hydroxylations and $17 C$-methylations) are indeed catalyzed by enzymes belonging to the cluster or whether additional genes might be located elsewhere on the symbiont genome. If the cluster is sufficient for biosynthesis, the putative $\mathrm{Fe}(\mathrm{II}) / \alpha$-ketoglutarate-dependent oxygenase PoyI and the two radical-SAM methyltransferase-like enzymes PoyC and PoyD would be candidates for these 23 transformations.

Prior to the discovery of the polytheonamide gene cluster, the existence of a new RiPP family with unusually long nitrile hydratase-like leader peptides (NHLP) sequences was postulated based on bioinformatic analyses. ${ }^{3}$ These sequences are found in genome sequences of diverse bacteria, although the identity of the peptides was unknown. This prediction demonstrates the power of in silico genome mining methods to discover new RiPP classes. In addition to NHLP homologues, a second precursor type that contains a leader region resembling Nif11 nitrogen-fixing proteins (N11P) was identified in the bioinformatic study. For both groups of leader peptide, one or multiple radical-SAM genes (predicted to encode amongst 
others, epimerases, and $C$-methyltransferases) are frequently present in the biosynthetic gene clusters. ${ }^{5}$ Thus, although polytheonamides are currently the only known characterized proteusins, a unifying feature of this family seems to be NHLP or $\mathrm{N} 11 \mathrm{P}$ sequences and modifications involving radical-SAM enzymes. Interestingly, the N11P-like leader peptides are also used to produce lanthipeptides in marine cyanobacteria., ${ }^{3,26}$ Radical-SAM proteins are also used for the biosynthesis of two other RiPP families, bottromycins and sactipeptides, and bioinformatic analyses suggest that these radical-SAM mediated transformations may be as common as the ubiquitous (cyclo) dehydration reactions discussed in sections $5-8 .^{5}$

\subsection{Specific recommendations for the proteusin family}

One common feature at the genetic level is the conspicuous and characteristic nitrile hydratase- or Nif11-like leader peptide of the precursor gene. Similar to other RiPPs it is recommended to use the descriptor " $A$ " for the precursor peptide. Regarding posttranslational modification, it is currently premature to propose unifying recommendations, since polytheonamides are the only members known to be associated with this family, and there is generally little overlap with the biosynthetic enzymes of other RiPP types.

\section{Linear azol(in)e-containing peptides (LAPs)}

\subsection{Historical perspective}

The earliest work on the linear azol(in)e-containing peptides (LAPs) dates back to at least 1901 with the observation that certain pathogenic isolates of streptococci secrete a factor responsible for what later became known as the $\beta$-hemolytic phenotype. ${ }^{164,165}$ Despite a flurry of work carried out on the hemolytic streptococci in the opening decade of the 20th century, studies in the 1930s provided the first significant insight into the $\beta$-hemolytic factor, streptolysin S (SLS). ${ }^{\mathbf{1 6 6 , 1 6 7}}$ Because of the problematic physicochemical properties of SLS, it was not until 2000 that researchers realized the defining chemical attributes of this RiPP subfamily. ${ }^{\mathbf{1 6 8}}$ This realization was enabled by a rapid advancement in the understanding of the biosynthesis of another LAP member, microcin B17, the first RiPP to have its biosynthesis reconstituted in vitro (vide infra). ${ }^{50,51}$ To this day, the exact chemical structure of SLS remains elusive. However, the classification of SLS as a LAP and an integral component of the pathogenic mechanism of Streptococcus pyogenes was confirmed by a series of recent genetic and biochemical studies. ${ }^{7,37,169}$ Bioinformatic and biochemical investigations have shown that a number of pathogens from the Firmicutes phylum contain SLS-like gene clusters, including lineage I strains of Listeria monocytogenes and Clostridium botulinum. ${ }^{\mathbf{1 7 0 - 1 7 2}}$

\subsection{LAP biosynthesis}

LAPs are decorated with various combinations of thiazole and (methyl)oxazole heterocycles, which can sometimes also be found in their corresponding 2-electron reduced azoline state
(Fig. 8). ${ }^{52}$ As demonstrated for microcin B17 in 1996, the azol(in)e heterocycles derive from cysteine, serine, and threonine residues of a ribosomally synthesized precursor peptide. ${ }^{51}$ The critical components in LAP biosynthesis are the inactive precursor peptide (referred to as "A") and the heterotrimeric synthetase complex comprised of a dehydrogenase (B) and cyclodehydratase (C/D). In many LAP biosynthetic gene clusters, the $\mathrm{C}-\mathrm{D}$ proteins are fused in a single polypeptide, highlighting the importance of their enzymatic collaboration..$^{52,173}$ The first step in LAP biosynthesis is substrate recognition, driven through an interaction of the $\mathrm{N}$-terminal leader peptide of the precursor (Fig. 8 and 9), which remains unmodified. ${ }^{37,58}$ After formation of the enzyme-substrate complex, ATP-dependent cyclodehydration occurs with the expulsion of water from the preceding amide carbonyl giving rise to an azoline heterocycle. ${ }^{173}$ ATP is used in this step to phosphorylate the amide oxygen to facilitate the elimination of a water equivalent from the substrate. ${ }^{173}$ In a second step, a flavin mononucleotide (FMN)-dependent dehydrogenase oxidizes a subset (sometimes all) of the azolines to the aromatic azole heterocycles. ${ }^{51,174} \mathrm{~A}$ typical LAP will then undergo proteolytic processing to remove the leader peptide with subsequent transport out of the producing cell by a dedicated ATP binding cassette (ABC) type transporter.

While the defining feature of a LAP is the genetically and biochemically conserved installation of azol(in)e rings on nonmacrocyclized natural products (as opposed to the cyanobactins and thiopeptides discussed in the following sections), other post-translational modifications are often found. These

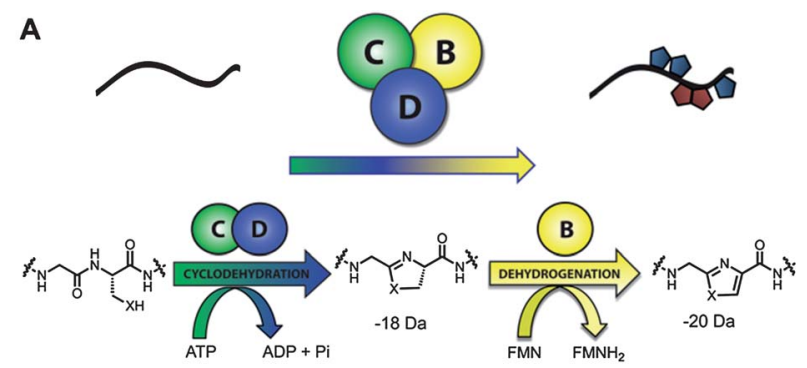

B

MLKFTSNILATSVAETTQVAPGG-CCCCCTTCCFSIATGSGNSQGGSGSYTPGK MELKASEFGVVLSVDALKLSRQSPLG-VGIG 10 SCGGQGGGCGGCSNGCSGGNGGSGGSGSHI MENVQTLAIDDIENIDAEVTIEELSSTNGA-ATVSTILCSGGTLSSAGCV MTQIKVPTALIASVHGEGQHLFEPMAA-RCTCTTIISSSSTF

Fig. 8 LAP biosynthetic scheme and precursor peptide sequences. A, Upon recognition of the precursor peptide (black line) by the heterocycle-forming $B C D$ enzymatic complex, select Cys and Ser/Thr undergo a backbone cyclodehydration reaction with a preceding amino acid. Catalyzed by a cyclodehydratase (C/Dproteins, green and blue), net loss of water from the amide carbonyl during this reaction yields an azoline heterocycle. Further processing by a FMN-dependent dehydrogenase (B-protein, yellow) affords the aromatic azole heterocycle. B, Amino acid sequence of several LAP precursors. Like most RiPPs, these consist of a leader peptide (to facilitate recognition by the modifying enzymes) and core region that encodes for the resultant natural product. From top to bottom are the LAP precursors for SLS, microcin B17, goadsporin, and plantazolicin. Post-translational modifications are color-coded (Cys resulting in thiazoles, red; Ser/Thr resulting in oxazoles and methyloxazoles, blue; Thr leading to methyloxazoline, brown; Ser converted to dehydroalanine, light blue; N-terminal acetylated/ methylated residues, green). The leader peptide cleavage site is indicated with a dash (predicted for SLS). 


\section{Microcin B17}
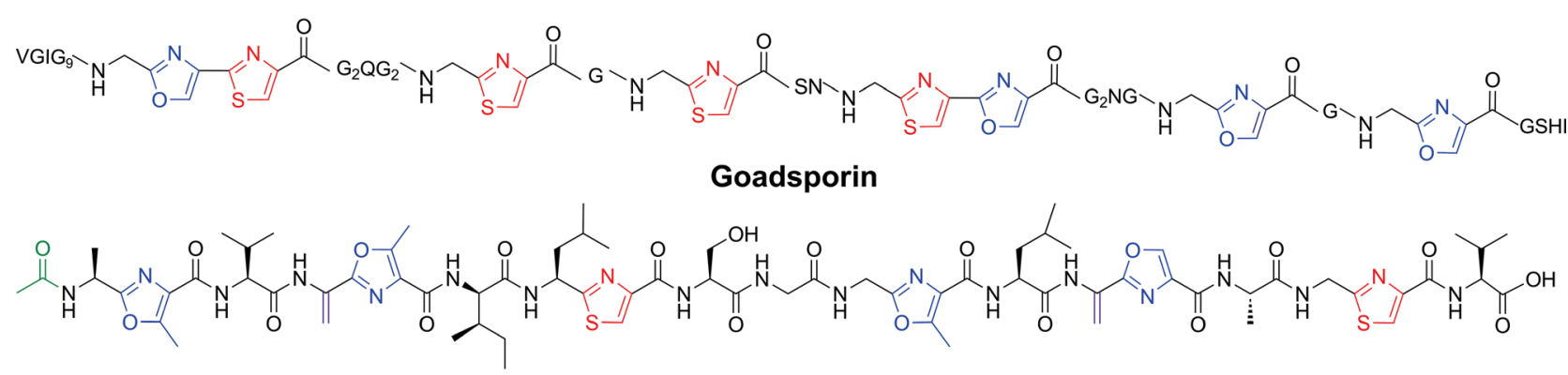

Plantazolicin

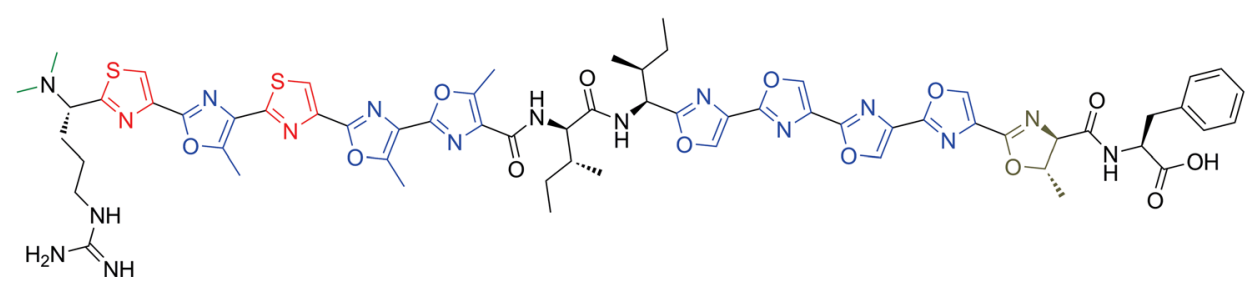

Fig. 9 Representative structures of LAPs. Microcin B17 is a DNA gyrase inhibitor (antibiotic), goadsporin induces secondary metabolism and morphogenesis in actinomycetes, and plantazolicin is a selective antibiotic with an unknown biological target. Color coding as in Fig. 8.

ancillary tailoring modifications include acetylation, methylation, and dehydration. , $^{74,52}$

\subsection{Structures and bioactivities of LAPs}

Aside from microcin B17 and SLS, a number of additional LAPS have been identified by mining publicly available genome sequences. Only those LAPs with a fully elucidated chemical structure are discussed here, but it is probable that trifolitoxin and some nitrile hydratase/nif11-derived natural products are also LAPs., ${ }^{\mathbf{3} 175,176}$ Goadsporin is produced by Streptomyces sp. TPA0584 and contains two thiazoles, four (methyl)oxazoles, two dehydroalanines, and an acetylated N-terminus (Fig. 9). ${ }^{177,178}$ The genes responsible for the chemical maturation of this LAP were reported in $2005 .{ }^{44}$ Goadsporin provides a link between the LAP family and the lanthipeptides as the dehydroalanines are generated by proteins with homology to the class I lanthipeptide dehydratases (LanBs). The precise biological target(s) of goadsporin has not yet been determined, but it is known to induce secondary metabolism and morphogenesis in a wide variety of streptomycetes. Goadsporin also exhibits antibiotic activity against Streptomyces, including potent activity against Streptomyces scabies, the causative agent of potato scab.

Plantazolicin (PZN) was predicted by bioinformatics to be an excreted metabolite from the soil-dwelling microbe Bacillus amyloliquefaciens FZB42. ${ }^{7}$ Genetic evaluation of the PZN biosynthetic pathway, molecular weight determination, and confirmation of antibiotic activity were reported in early $2011 .{ }^{179}$ Later that year, two groups independently reported the structure of PZN. ${ }^{180,181}$ Cyclodehydration was shown to precede dehydrogenation in vivo, ${ }^{\mathbf{1 8 1}}$ as hypothesized from earlier work on microcin B17 and azol(in)e-containing cyanobactins. ${ }^{\mathbf{5 1 8 2 - 1 8 4}}$ PZN has striking antimicrobial selectivity for Bacillus anthracis, and its biosynthetic genes are found in several other Grampositive bacterial species. ${ }^{\mathbf{1 8 1}}$

\subsection{Relationship to other RiPP subfamilies}

The genes responsible for installing the azol(in)e heterocycles are the defining characteristic of the LAP subfamily of RiPPs. It is important to note that the BCD heterocycle-forming genes are genetically similar with respect to biochemical function to those that install heterocycles on macrocyclized RiPPs (e.g. the thiopeptides, cyanobactins, and bottromycins, vide infra). ${ }^{50,52,185}$

\subsection{Specific recommendations for the LAP family}

To avoid future confusion with the letter assignment of biosynthetic genes for LAPs, we recommend that "A" always be reserved for the precursor peptide, regardless of its location within a biosynthetic gene cluster. Likewise, we encourage the use of "C/D" for the cyclodehydratase and "B" for clusters that encode a dehydrogenase.

\section{Cyanobactins}

\subsection{Structures and biosynthesis}

In the 1970s, growing interest in bioactive components of marine animals prompted Ireland and Scheuer to investigate the tunicate Lissoclinum patella, collected on tropical coral reefs in the Western Pacific. In 1980, they reported the structure elucidation of two small, cytotoxic peptides, ulicyclamide and ulithiacyclamide, revealing a then-unprecedented combination of chemical features, including $\mathrm{N}$-to-C macrocyclization and heterocyclization to form thiazol(in)e and oxazoline motifs (Fig. 10). ${ }^{186}$ L. patella harbors an abundant, uncultivated symbiotic cyanobacterium, Prochloron didemni, leading the authors to propose that symbiotic cyanobacteria make the compounds. Indeed, 12 years after this initial report, the homologous compound westiellamide was isolated from cultivated cyanobacteria and from ascidians. ${ }^{187,188}$ Overall, 

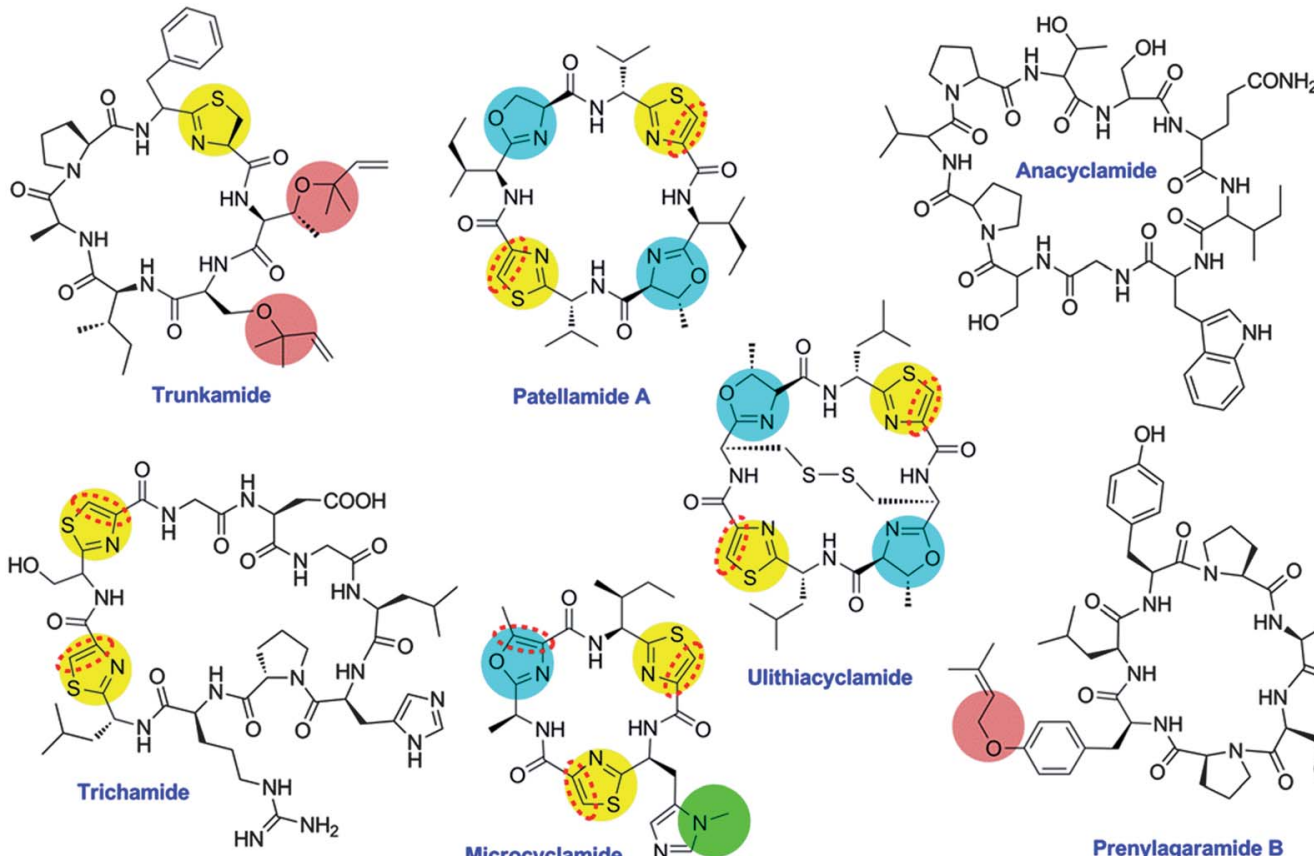

Patellamide A

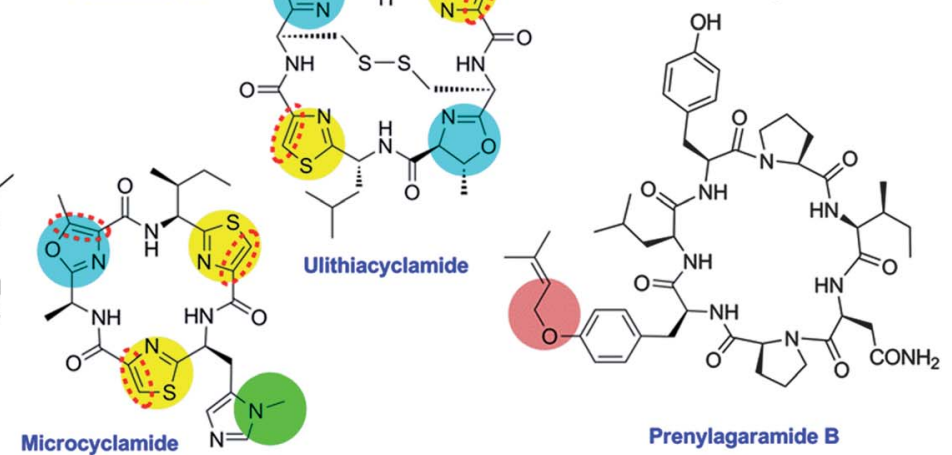

Fig. 10 Representative cyanobactins. Anacyclamide is modified only by N-C cyclization, while others are modified by heterocyclization of Cys (yellow) or Ser/Thr (blue), oxidation to azole (red dashed line), prenylation (pink) or $\mathrm{N}$-methylation (green).

approximately 100 related compounds were described from marine animals and cultivated cyanobacteria as of the early 2000s. ${ }^{185}$ Compounds in the group were N-to-C macrocyclic, and many (but not all) were further modified by heterocyclization. Several family members were prenylated on Ser, Thr, or Tyr, while others were $N$-methylated on His.

The family relationship of these structurally and functionally diverse metabolites was not universally appreciated until their gene clusters were sequenced. Genes were reported for the patellamide group in $2005^{21}$ and for ulithiacyclamide itself in $2006,{ }^{25}$ confirming that the "animal" compounds were in fact produced by $P$. didemni. ${ }^{21,189}$ Biosynthetic genes from many different free-living and symbiotic cyanobacteria have since been reported, leading to the proposal for a family name: cyanobactins (Fig. 10). ${ }^{32,64,190-195}$ Cyanobactins are defined as N-C macrocyclic peptides encoded on a precursor peptide (designated as "E", as in PatE in patellamide biosynthesis), with proteolytic cleavage and macrocyclization catalyzed by homologous serine proteases "A" and "G", as in PatA and PatG. ${ }^{32,185,196}$ Optional, but widely-occurring, cyanobactin biosynthetic proteins include "D", as in PatD, the cyclodehydratase from the patellamide pathway; " $\mathrm{F}$ ", as in LynF, the tyrosine prenyltransferase from the lyn pathway; and conserved proteins of unknown function ("B" and "C"). Additional elements include thiazoline/oxazoline dehydrogenases that aromatize the heterocycles to thiazoles and oxazoles, methyltransferases, and several other uncharacterized proteins. Of note, the D cyclodehydratase consists of the C-D domains found in LAP biosynthesis (vide supra), and the azoline dehydrogenase is often, although not always, part of the G protein and is homologous to the dehydrogenase referred to as "B" in the LAP family.
About 200 cyanobactins have been identified, mostly via traditional chemical methods but at least one-third by genome mining. ${ }^{64,185,196}$ All known cyanobactins derive from cyanobacteria (or marine animals, where a cyanobacterial origin is likely) and are between 6-20 amino acids in length. Phylogenetic analysis has grouped the gene clusters into several families that are predictive of the resulting chemical structures. ${ }^{64}$ Other types of N-to-C macrocyclic peptides (circular peptides) abound in nature and include the cyclotides and orbitides from plants, ${ }^{197,198}$ the amatoxins and phallotoxins from fungi, ${ }^{22,199}$ and circular bacteriocins ${ }^{200}$ among others (see their respective sections in this review). However, these compounds are so far not known to be produced using PatA, PatG, and PatE-like proteins, and they contain different secondary modifications. Cyanobactin gene clusters have been identified only in cyanobacteria to date, and evidence suggests that they might be present in $\sim 30 \%$ of cyanobacterial strains. ${ }^{191,192}$ However, it is likely that cyanobactins exist in other organisms as well. For example, structurally related peptides such as telomestatin are well known from Streptomyces spp. These compounds are headto-tail cyclized and contain heterocycles, but, in contrast to known cyanobactins, multiple heterocycles are found in tandem, some of which derive from non-proteinogenic amino acids such as $\beta$-hydroxytyrosine. Recently, a gene cluster for the telomestatin family compound YM-216391 was reported. ${ }^{201}$ Interestingly, while YM-216391 is made by LAP-like cyclodehydratases, the route to macrocyclization is convergent and utilizes different machinery.

Biosynthesis of cyanobactins begins with the E-peptide, which contains at minimum a leader peptide, an $\mathrm{N}$-terminal protease recognition sequence, a core peptide, and a C-terminal recognition sequence (Fig. 11). ${ }^{21}$ Both recognition sequences 

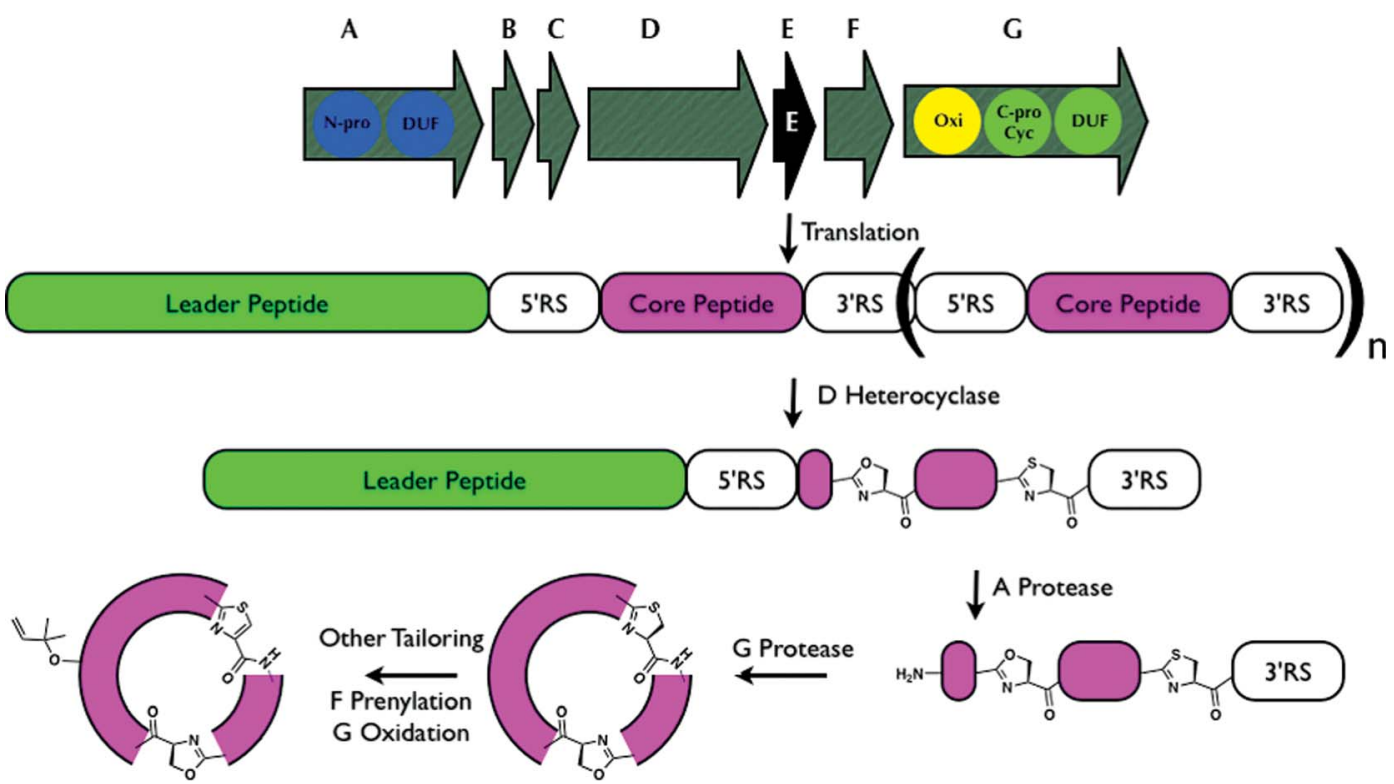

Fig. 11 Generalized biosynthesis of cyanobactins that contain azoline heterocycles. Translation of the core peptide is followed by heterocyclization, then proteolytic tailoring to yield macrocycles. RS = recognition sequence, essential for proteolytic tailoring. Multiple copies of core peptide + recognition sequences are possible; here an example is shown in which a single core peptide exists and is modified $(n=0)$. Other tailoring, such as prenylation, probably takes place on the mature cycle, while the timing of oxidation / dehydrogenation to azoles is not known. Additional modifications are also sometimes present. ${ }^{185} \mathrm{Oxi}=$ thiazoline oxidase, $\mathrm{N}$-pro and C-pro are the protease domains that cleave the N-terminus and C-terminus of the core peptides, respectively, and DUF are domains of unknown function. C-pro also performs macrocyclization (Cyc).

are generally 4-5 amino acids in length. Often, more than one core peptide (up to 3 thus far) is encoded on a single E-gene; in these cases, each core peptide is flanked by conserved $\mathrm{N}$ - and $\mathrm{C}$ terminal recognition sequences. ${ }^{64}$ If a D-protein (cyclodehydratase) is present, this protein acts first by regioselective modification of Cys, Ser, and/or Thr residues to form oxazoline and thiazoline. ${ }^{\mathbf{1 8 3 , 1 8 4}}$ The E-peptide substrate is helical, ${ }^{60}$ presumably facilitating its interaction with $\mathrm{D},{ }^{58}$ and a short sequence element in the $\mathrm{E}$ leader peptide is diagnostic of heterocycle formation. ${ }^{64}$ Subsequently, other modifications take place. The A protease clips off the $\mathrm{N}$-terminal recognition sequences, leaving a free amine for macrocyclization. ${ }^{53}$ The $\mathrm{G}$ protease then cleaves at the C-terminal recognition sequence and catalyzes $\mathrm{C}-\mathrm{N}$ macrocyclization. ${ }^{53,202}$ An X-ray structure of the macrocyclase domain of PatG demonstrated that it has a subtilisin-like fold but contains insertions not found in these proteases. $^{203}$ Furthermore, the structure of an acyl enzyme intermediate in which the peptide to be cyclized is bound to an active site Ser residue provides insights into the macrocyclization process. ${ }^{203}$ At present, it appears that prenylation occurs after macrocyclization, and only if a certain type of $\mathrm{F}$ gene is present. ${ }^{204}$ The F-protein is usually specific for dimethylallylpyrophosphate, ${ }^{204}$ but evidence suggests that longer isoprenoids can be incorporated in some cyanobactins. ${ }^{192}$ When multiple core peptides are present on a single E-precursor, they are all modified into cyanobactin products, ${ }^{21}$ and multiple E genes are often found in single organisms or pathways. ${ }^{64}$ Cyanobactins often contain D-amino acids, which arise spontaneously because of their adjacency to azol(in)e residues. ${ }^{32,205}$ Like the biosynthetic enzymes discussed for many other compound classes in this review, the cyanobactin biosynthetic enzymes are promiscuous and accept diverse proteinogenic and non-proteinogenic substrates in vivo and in vitro. ${ }^{25,202,204,206}$

Cyanobactins represent an enormous array of sequence and functional group diversity, and many different biological activities have been attributed to the molecules, including anticancer, antiviral, anti-protease, and antibiotic activity. ${ }^{\mathbf{1 8 5 , 2 0 7}}$ Notably, several compounds exhibit selective toxicity to certain human cancer cell lines, ${ }^{207,208}$ some potently block the human multidrug efflux pump, P-glycoprotein, ${ }^{209,210}$ and many cyanobactins are known for their ability to bind transition metals..$^{207,211,212}$

\subsection{Specific recommendations for the cyanobactin family}

It is recommended that the nomenclature for proteins in this group be based upon the precedent of the patellamide pathway. Therefore, the defining peptides are precursor peptide (E), Nterminal protease (A), and C-terminal protease (G). Other commonly occurring proteins in this group include cyclodehydratase (D), prenyltransferase (F), and hypothetical proteins (B and $\mathrm{C}$ ).

\section{Thiopeptides}

Thiopeptides were first reported in the late 1940s with the isolation of a micrococcin, ${ }^{213}$ and have since burgeoned into a family of roughly 100 metabolites, a few of which are represented in Fig. 12A. To date, the majority of thiopeptides are produced by Actinobacteria, but several have been obtained 


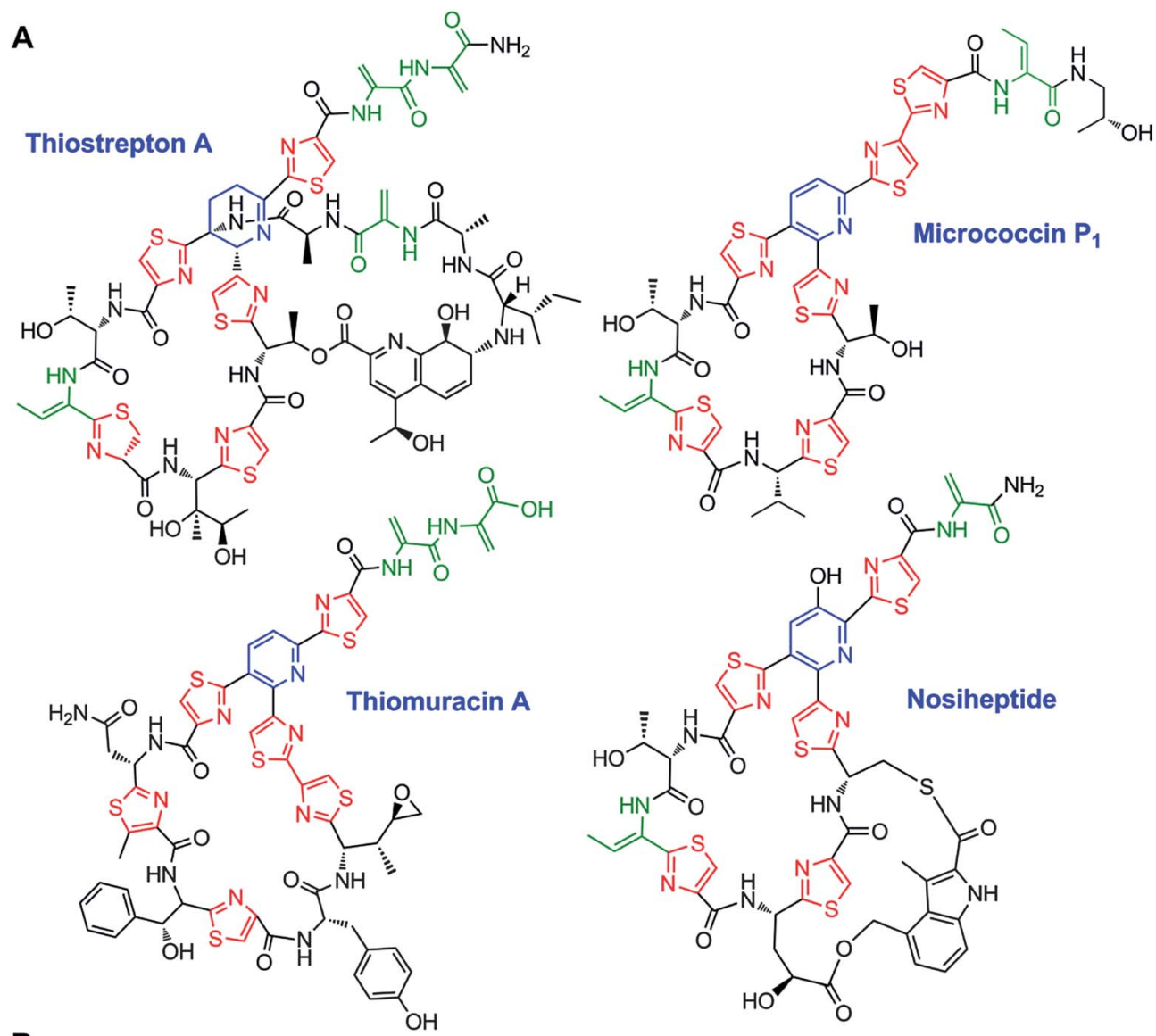

B<smiles>[R3]C1CCC([R])([R4])[C@H]([R2])N1</smiles>

Series a<smiles>[R9]C1=N[C@H]([R2])C([R4])([R])CC1</smiles>

Series $b$<smiles>[R2]C(=O)C1(CO)C=C(C)C2CCC([R2])([R4])[C@H]([R2])N21</smiles>

Series $c$<smiles>[R]c1ccc([R])c([R])n1</smiles>

Series $d$<smiles>[R2]c1cc(O)c([R3])nc1[R2]</smiles>

Series e

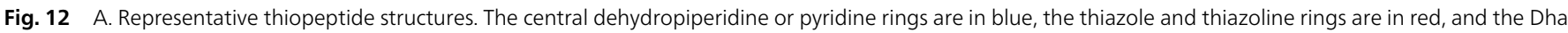
and Dhb residues are in green. B. The structural series of thiopeptides, showing the typical substitution pattern of the central nitrogenous heterocycle.

from Firmicutes, including those from the Bacillus and Staphylococcus genera. ${ }^{\mathbf{2 1 4 - 2 1 6}}$ Also referred to as thiazolyl peptides or pyridinyl polythiazolyl peptides, the thiopeptides typically feature a highly modified peptide macrocycle bearing several thiazole rings and often possess multiple dehydrated amino acid residues (for a review, see ${ }^{214}$ ). A defining feature of the thiopeptide macrocycle is a six-membered nitrogenous ring that can be present in one of three oxidation states: a piperidine, dehydropiperidine, or pyridine. Further architectural complexity is achieved in some thiopeptides by the addition of a second macrocycle to incorporate a tryptophan-derived quinaldic acid or an indolic acid residue, such as those found in thiostrepton A and nosiheptide (Fig. 12A). ${ }^{217,218}$ For over sixty years following the discovery of micrococcin, it was uncertain whether thiopeptides derived from the extensive post-translational modification of ribosomally-synthesized precursor peptides, or, instead, if their polypeptide backbones were the products of nonribosomal peptide synthetases. A series of publications in early 2009 revealed the biosynthetic gene clusters of five thiopeptides, leading to the formal recognition that these metabolites are indeed RiPPs. ${ }^{46-49}$

\subsection{Structural classifications of thiopeptides}

Structurally, the thiopeptides can be classified into five series, based on the oxidation state and substitution pattern of the central nitrogenous heterocycle (Fig. 12B). This classification scheme originates from an initial proposal by Hensens in 1978, and was later elaborated by Bagley to accommodate a more diverse set of metabolites - nearly 80 members by $2005 .^{214,219}$ Series $a$ thiopeptides, exemplified by certain thiopeptins, present the core heterocycle as a fully saturated piperidine. ${ }^{219,220}$ Series $b$ is classically represented by thiostrepton A and its dehydropiperidine ring. ${ }^{221}$ Series $c$, thus far, is comprised of a single entry, Sch 40832, in which the core nitrogen heterocycle is contained within an imidazopiperidine. ${ }^{222}$ The trisubstituted pyridine ring, in micrococcin $\mathrm{P}_{1}$ and other series $d$ metabolites, is the most frequently encountered scaffold among the 
thiopeptides. ${ }^{\mathbf{2 1 4 , 2 2 3 , 2 2 4}}$ The final sub-family, series $e$, also contains a pyridine, but possesses a hydroxyl group at the fifth position of the ring, as can be observed in nosiheptide (Fig. 12A). ${ }^{225,226}$ All members of the series $a-c$ thiopeptides contain a second macrocycle in which a quinaldic acid, 4-(1hydroxyethyl)quinoline-2-carboxylic acid, links one side chain from the core macrocycle to the core peptide N-terminus (e.g. thiostrepton A, Fig. 12A). In an analogous fashion, series $e$ metabolites also harbor a second macrocycle, with the distinction that two of the core macrocycle's side chains are connected to an indolic acid residue.

\subsection{Biological activities of thiopeptides}

Varied biological activities are attributed to thiopeptides, yet these RiPPs are perhaps best known for their antibacterial properties. Although thiopeptides are potent inhibitors of Gram-positive bacterial growth, little effect is generally exerted upon Gram-negative bacteria. This spectrum of antibacterial activity is attributed to an inability of thiopeptides to penetrate the outer membrane of Gram-negative species, since in vitro studies of thiopeptide function often employ the biochemical

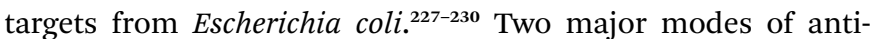
bacterial action are commonly reported for the thiopeptides, both of which impede protein synthesis. The first, observed of thiostrepton $\mathrm{A}$, nosiheptide, micrococcin $\mathrm{P}_{1}$, and several others, results from a direct interaction with the prokaryotic $50 \mathrm{~S}$ ribosomal subunit. ${ }^{231-233}$ These thiopeptides bind near the GTPaseassociated center that engages a number of translation factors during initiation and elongation. ${ }^{234-236}$ Structural studies revealed that thiopeptides bind to a cleft between the ribosomal protein L11 and the 23S rRNA, and the precise intermolecular interactions differ according to the exact structural features of the thiopeptide. ${ }^{231,237}$ Complexation of the ribosome and a thiopeptide disrupts the conformational changes ordinarily communicated from the translation factors to the ribosome, ultimately halting translocation along the mRNA

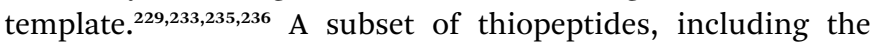
thiomuracins and GE2270A, exert a separate mode of antibacterial action by the inhibition of elongation factor Tu (EF-Tu). EF-Tu ordinarily delivers aminoacylated tRNAs to the ribosome following translocation. ${ }^{49,228}$ When a thiopeptide binds to EF$\mathrm{Tu}$, a portion of the recognition site for the aminoacyl tRNA substrate is occluded and formation of an EF-Tu-aminoacyltRNA complex is prevented. $228,238,239$

In addition to their well-known antibacterial properties, certain thiopeptides have demonstrated antimalarial and anticancer activities. For both of these latter cases, it appears that the mode of action may be two-fold. Thiostrepton A inhibits two opposing aspects of protein homeostasis in the malaria parasite Plasmodium falciparum: the proteasome and protein synthesis within the parasite's specialized apicoplast organelle. ${ }^{240-243}$ In cancer cell lines, thiostrepton A appears to induce apoptosis through the interplay of proteasome inhibition and direct interference with the forkhead box M1 (FOXM1) transcription factor. ${ }^{\text {244-246 }}$ Other activities ascribed to thiopeptides span a range that encompasses RNA polymerase inhibition, renin inhibition, and some of these metabolites are proposed to serve as streptomycete signal molecules. ${ }^{24-250}$ The challenges now remain to decipher which aspects of the thiopeptide structure are key for each biological target, and then to apply that knowledge toward the development of novel anti-infective and anti-cancer chemotherapies.

\subsection{Biosynthesis of thiopeptides}

At the time of this review's preparation, ten thiopeptide biosynthetic gene clusters have been identified. ${ }^{46-49,251-255}$ In addition to the precursor peptide (e.g. TpdA of the thiomuracin cluster), at least six proteins, corresponding to TpdB-G, are found within each cluster. These six proteins presumably provide the minimal set of post-translational modifications required to construct the defining thiopeptide scaffold. ${ }^{49}$ The thiopeptides harbor structural features similar to both lanthipeptides (Dha and Dhb residues) and the linear azol(in)e-containing peptides (LAPs) and cyanobactins (thiazole structures). ${ }^{50,52}$ Not surprisingly, these backbone modifications are installed by similar enzymes. Dehydration of Ser and Thr side chains to introduce Dha and Dhb residues, respectively, is likely effected by one or two proteins, TpdB and TpdC, each bearing weak similarity to the class I lanthipeptide LanB dehydratases. ${ }^{\mathbf{9 0}, 91}$ Proteins similar to the cyclodehydratases (TpdG) and dehydrogenases (TpdE) that construct the polyazoline rings of the cyanobactins and LAPs are predicted to perform in a similar context for thiopeptide maturation..$^{21,44,179}$ Cyclization of a linear peptide is expected to provide the central pyridine/dehydropiperidine/piperidine of the thiopeptides, and Bycroft and Gowland suggested this could ensue through a $[4+2]$ cycloaddition of two dehydroalanines (Fig. 13). ${ }^{223}$ Inactivation of $t c l M$, a $t p d D$ homolog, in the thiocillin-producing Bacillus cereus strain led to the accumulation of a highly modified and linear peptide that contains the two anticipated dehydroalanine resides. ${ }^{256}$ The identification of this metabolite lends support to the Bycroft-Gowland proposal and implicates a key role for TclM/ TpdD and their homologs in this biochemically intriguing transformation. At present, it remains to be determined whether this cycloaddition proceeds in a concerted or stepwise process. The sixth protein found in all thiopeptide biosynthetic systems, TpdF, does not display any significant similarity to proteins of known function, and its biochemical role awaits elucidation. Like other RiPPs discussed in this review, the biosynthetic machinery of thiopeptides is tolerant of many mutations in their linear

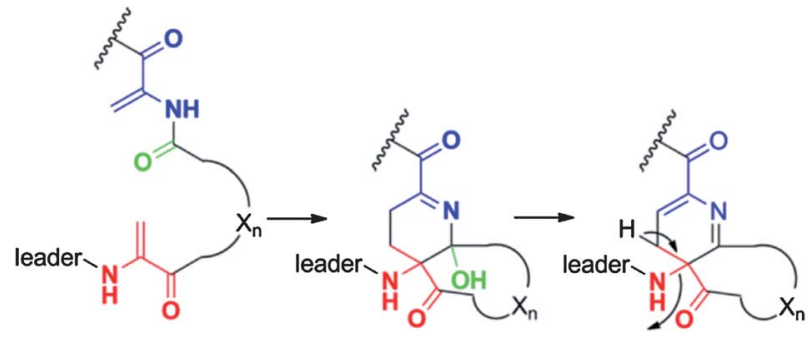

Fig. 13 Bycroft proposal for generation of the pyridine ring in thiopeptides from two Dha residues. The aromatization step would also remove the leader peptide. $^{\mathbf{5 0}}$ 
substrate peptides resulting in new variants. ${ }^{36,38,42,43}$ However, thiopeptides from the $d$ and $e$ series may be unique among RiPPs in the manner by which the leader peptide is likely removed. Rather than relying on proteolytic cleavage, the leader peptide is thought to be eliminated from the central nitrogen-containing heterocycle (Fig. 13). ${ }^{50}$ Beyond the conserved six proteins, one or more additional enzymes are embedded within each cluster to introduce the specialized features of each metabolite, including hydroxylations, methylations, and incorporation of an indolic or quinaldic acid macrocycle. .6,49,255,257 $^{4}$

\subsection{Specific recommendations for the thiopeptide family}

Given the overlap in nomenclature used between the LAP, cyanobactin, and lanthipeptide modification enzymes and the current lack of a common naming scheme, a thiopeptidespecific nomenclature for the core set of biosynthetic proteins seems appropriate. To be consistent with the bulk of RiPP gene nomenclature, it is recommended that the precursor peptide is annotated as "A". It is also suggested that future annotations of thiopeptide gene clusters follow the nomenclature outlined for the thiomuracins, wherein the precursor peptide and the core set of six genes were collinearly organized. ${ }^{49}$ By adopting this nomenclature, proteins corresponding to the dehydratases will be designated as " $\mathrm{B}$ " and " $\mathrm{C}$ "; the azoline-synthesizing dehydrogenase and cyclodehydratase are to be designated as " $\mathrm{E}$ " and "G", respectively; "D" is to be used for the protein implicated in the putative $[4+2]$ cycloaddition; and "F" should be assigned for the remaining conserved protein. Although this annotation may lead to discontinuous name assignment for a cluster's components, since thiopeptide clusters do not all follow this gene organization, it will ultimately permit a uniform notation for the core set of thiopeptide modification enzymes.

\section{Bottromycins}

The first member of a series of related compounds called bottromycins was originally isolated from the fermentation broth of Streptomyces bottropensis DSM 40262, ${ }^{258}$ but their structures were not definitively known until a total synthesis of bottromycin A2 in 2009 that settled a series of structural revisions (Fig. 14). ${ }^{259}$ These compounds contain a number of unique structural features including a macrocyclic amidine and a

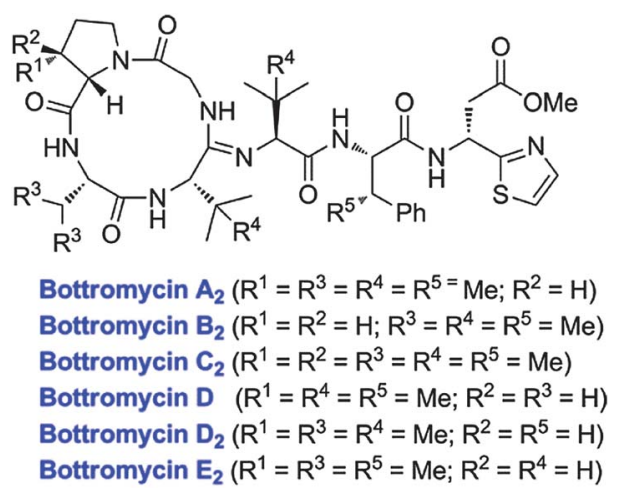

Fig. 14 Structures of various bottromycins. decarboxylated C-terminal thiazole. In addition, like the proteusins (section 4), they contain a series of C-methylated amino acids (Fig. 14). The bottromycins display potent antimicrobial activity against methicillin-resistant Staphylococcus aureus (MRSA) and vancomycin-resistant enterococci (VRE), ${ }^{\mathbf{2 6 0}}$ and function by blocking aminoacyl-tRNA binding to the A-site of the bacterial 50S ribosome. ${ }^{261-263}$ Recently, using various genome mining and genome sequencing methods, four studies reported that the bottromycins are RiPPs arising from an impressive series of modifications. ${ }^{17-20}$ The biosynthetic gene clusters were obtained by shot-gun sequencing of the genomes for Streptomyces sp. BC16019 17 and S. bottropensis ${ }^{18,19}$ that both were known to produce bottromycin $\mathrm{A}_{2}, \mathrm{~B}_{2}$, and $\mathrm{C}_{2}$ (Fig. 14). ${ }^{264-267}$ In an alternative strategy, a search of the nonredundant databases for a sequence encoding a putative linear core peptide GPVVVFDC provided a hit in the sequenced genome of the plant pathogen Streptomyces scabies 87.22, which was subsequently shown to produce bottromycins $\mathrm{A}_{2}, \mathrm{~B}_{2}$, and $\mathrm{C}_{2} \cdot{ }^{18,19}$ And in a final approach, a new analog, bottromycin $\mathrm{D}$ (Fig. 14) was detected in a metabolomics study of the marine ascidian-derived Streptomyces sp. WMMB272; subsequent genome sequencing revealed its biosynthetic gene cluster. ${ }^{20}$

\subsection{Biosynthesis of the bottromycins}

All four studies report the intriguing observation that the precursor peptide to the bottromycins does not contain an $\mathrm{N}$ terminal leader peptide, but instead a C-terminal extension of 35-37 amino acids (Fig. 15). The exact role of this extension is at present unclear, but it likely guides the biosynthetic enzymes. It is significantly larger than the C-terminal recognition sequences used for $\mathrm{N}$-to-C cyclization in cyanobactins (section 6), amatoxins (section 14), cyclotides (section 15), and orbitides (section 16), and because it likely functions in a role more similar to the leader peptides, the term follower peptide was introduced. ${ }^{19}$ Bioinformatics analysis has been used to predict the function of some of the genes that are found in the gene clusters of all four strains, which are highly similar in organization and sequence. ${ }^{17-20}$ Three putative cobalamin-dependent class $\mathrm{B}^{\mathbf{1 6 2}}$ radical-SAM methyltransferases were predicted to catalyze the methylation at the non-nucleophilic $\beta$-carbons. The Cys constellation in these enzymes (CxxxxxxxCxxC) ${ }^{\mathbf{1 8}}$ is different from the canonical CxxxCxxC motif involved in $\mathrm{Fe}-\mathrm{S}$ clusters in most radical-SAM proteins, but is also found in the polytheonamide $C$-methyltransferases PoyB and PoyC (section 4). The predicted function of these radical-SAM methyltransferases has been experimentally validated by gene deletion experiments in a heterologous expression system using Streptomyces coelicolor A3(2), ${ }^{17}$ and by gene deletions in $S$. scabies. ${ }^{18}$ One of the enzymes is responsible for $C$-methylation of the Pro residue as its deletion resulted in the exclusive production of bottromycin $\mathrm{B}_{2} \cdot{ }^{17,18}$ Similarly, a second radical-SAM methyltransferase is specific for the $C$-methylation of the Phe residue as its disruption resulted in the production of a new metabolite, bottromycin $\mathrm{D}_{2} \cdot{ }^{17}$ Deletion of the third methyltransferase resulted in the production of a compound that lacked two methyl groups, ${ }^{17,18}$ which was identified by tandem MS as bottromycin 

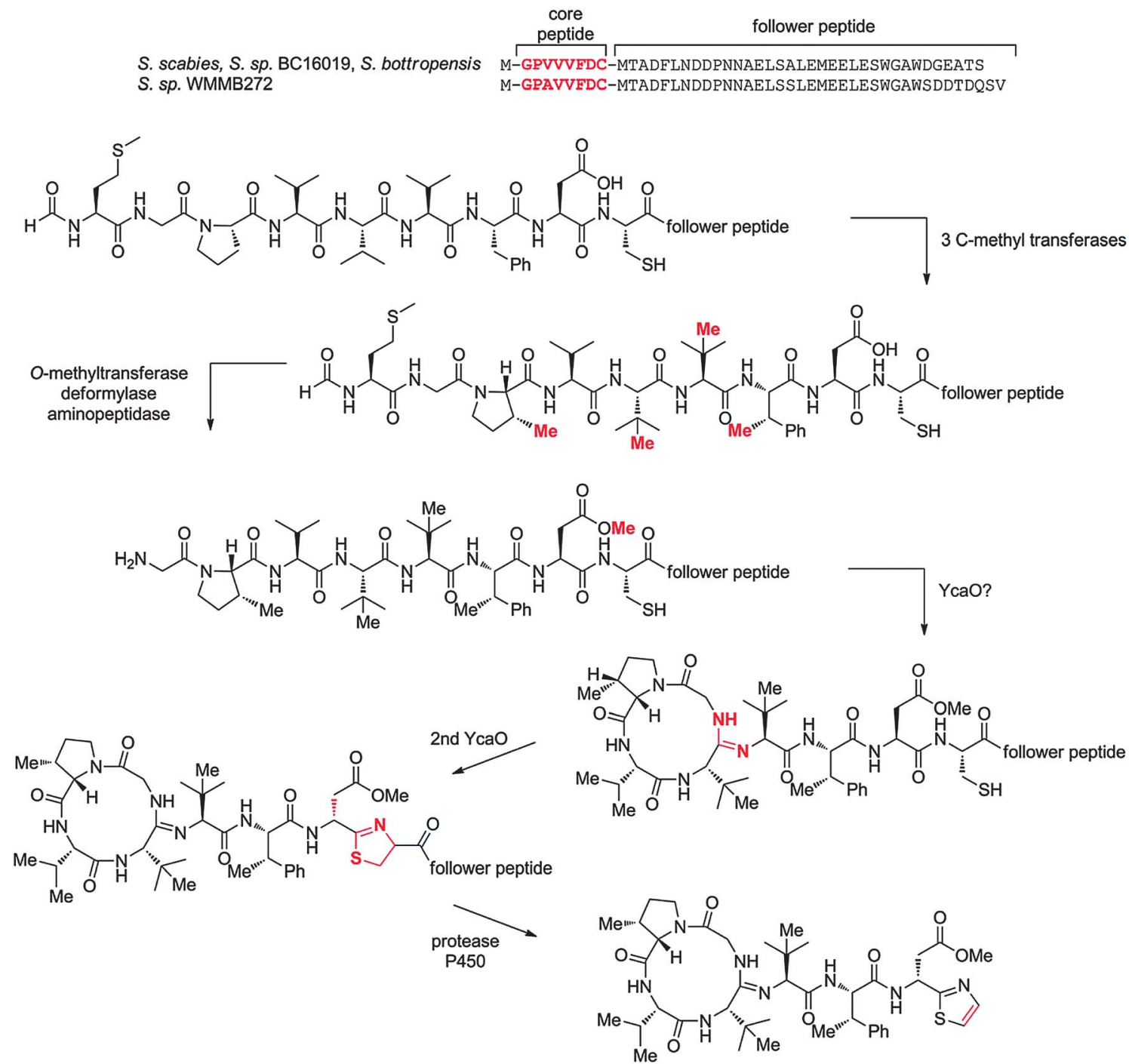

Bottromycin $\mathrm{A}_{2}$

Fig. 15 Potential biosynthetic pathway to bottromycin $A_{2}$. The order of the posttranslational modifications is currently not known and hence the order depicted is arbitrary. The precursor peptide sequences for the currently known bottromycins are shown at the top. Variations in the posttranslational modifications morph these precursor sequences into the structures shown in Fig. 14

$\mathrm{E}_{2}$ (Fig. 14). ${ }^{17}$ Hence, this third methyltransferase adds methyl groups to two of the three original Val residues of the precursor peptide (Fig. 15). In addition to the radical-SAM methyltransferases, the gene clusters also contain an $O$-methyltransferase that was shown to be responsible for the methylation of the Asp residue (Fig. 15). ${ }^{17}$

The gene clusters also contain two genes with a YcaO-like domain similar to the D-protein involved in LAP biosynthesis (section 5). This domain was shown for LAPs to use ATP to phosphorylate the amide backbone during the cyclodehydration reaction, ${ }^{173}$ and hence at least one of the two YcaOlike genes is predicted to be involved in thiazoline formation. ${ }^{17-20}$ Interestingly, the bottromycin gene cluster does not contain the C-protein that together with the D-protein promotes efficient cyclodehydration in LAPs (section 5) or cyanobactins (in which the $\mathrm{C}$ and $\mathrm{D}$ proteins are fused in one protein, see section 6). Why two YcaO-domain containing proteins are encoded in the bottromycin gene cluster is at present unclear, but one possibility is that one is involved in cyclodehydration to form a thiazoline and the second in the macrocyclodehydration to form the amidine structure (Fig. 15). ${ }^{17-19}$

Two genes encoding putative proteases/hydrolases and one gene encoding a putative aminopeptidase may be involved in removal of the follower peptide and the N-terminal Met, and possibly to aid formation of the unique macrocyclic amidine. Finally, a gene encoding a cytochrome $\mathrm{P} 450$ has been suggested to facilitate the oxidative decarboxylation of the C-terminal residue by hydroxylating the $\beta$-carbon of the Cys. ${ }^{19}$ The observed epimerization at the Asp $\alpha$-carbon may possibly occur nonenzymatically as a consequence of its location next to a thiazole/ thiazoline as has also been suggested for cyanobactins (section 6). ${ }^{32,205}$ The order of this impressive array of posttranslational 
modifications is currently not known and one possibility out of many is shown in Fig. 15. A putative bottromycin-transporter was also identified in the biosynthetic gene cluster, ${ }^{17-20}$ and proposed to be involved in the export of and self-resistance to bottromycins. This hypothesis was verified by an overexpression experiment with the Streptomyces coelicolor A3(2) heterologous expression system, which led to a 20 fold increase of bottromycin production yields compared to a parallel experiment using the unmodified pathway. ${ }^{17}$

\subsection{Specific recommendations for the bottromycin family}

It is recommended to use the term follower sequence for the unique C-terminal extension found in the bottromycin precursor peptides. Since four independent studies all used different gene annotations, at present a common gene nomenclature is not in use. In line with the recommendations for most other RiPPs, the use of the designation A is recommended for the gene encoding the precursor peptide in biosynthetic gene clusters that are uncovered in future studies.

\section{Microcins}

Historically, the name microcins has been used for ribosomally synthesized antimicrobial peptides from Enterobacteriaceae, mainly Escherichia coli. In addition to their lower molecular masses $(<10 \mathrm{kDa})$, microcins differentiate from colicins, the larger antibacterial proteins $(30-80 \mathrm{kDa})$ produced by Gramnegative bacteria, by a resistance to proteases, extreme $\mathrm{pH}$ and temperature, and in many cases the presence of post-translational modifications. Fourteen representatives have been identified thus far (for reviews see $^{\mathbf{1 2 , 2 6 8 , 2 6 9}}$ ) and eight of them have been isolated and structurally characterized (e.g. Fig. 16).

Most microcins are plasmid-encoded peptides. Their biosynthetic clusters display a conserved general organization, with (i) a structural gene encoding the precursor peptide, (ii) a self-immunity gene that protects the producing strain against its own toxic peptide, (iii) genes encoding the microcin export system, and (iv) often several genes encoding auxiliary proteins or modification enzymes. The classification of microcins adopted presently distinguishes two classes. $^{\mathbf{1 2 , 2 7 0}}$ Class I consists of three plasmid-encoded peptides with a molecular mass below $5 \mathrm{kDa}$ that have extensive backbone post-translational modifications: microcins B17, C (also called C7, C51 or C7/C51), and J25. Two of these compounds, microcins B17 and J25 were initially believed to be unique to Enterobacteria, but the genome sequencing efforts have demonstrated that they are part of larger families of structurally related peptides that are produced by a variety of bacteria. As described in the section on the LAP family, microcin B17 is a 43 amino acid peptide that carries four thiazole and four oxazole heterocycles (Fig. 9), which result from the modification of six glycines, four serines and four cysteines in the core peptide of the 69 amino acids long microcin precursor peptide (Fig. 8). ${ }^{271}$ Microcin C is an $N$-formylated heptapeptide covalently linked to adenosine monophosphate via its C-terminal aspartate through an $\mathrm{N}$-acyl phosphoramidate linkage; ${ }^{272}$ the phosphate group is further

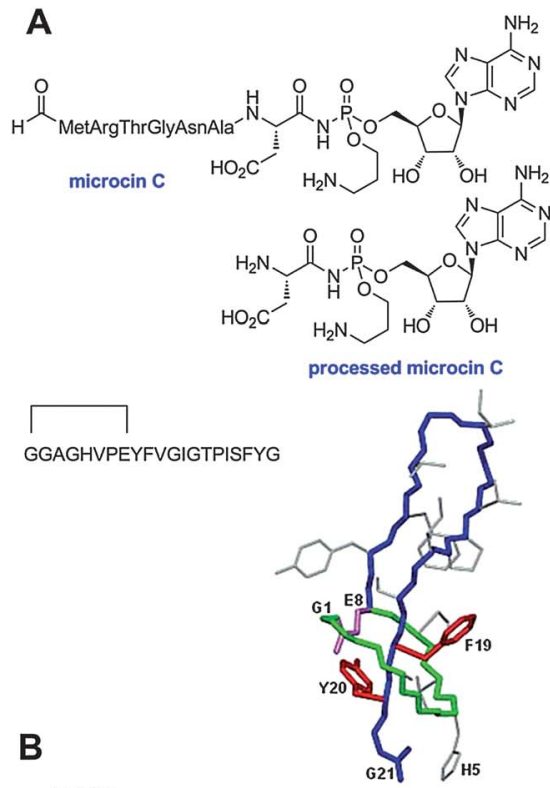

E492

GETDPNTQLL NDLGNNMAWG AALGAPGGLG SAALGAAGGA LQTVGQGLID HGPVNVPIPV LIGPSWNGSG SGYNSATSSS GSGS

M

DGNDGQAELI AIGSLAGTFI SPGFGSIAGA YIGDKVHSWA TTATVSPSMS PSGIGLSSQF GSGRGTSSAS SSAGSGS

$\mathrm{H} 47$

GGAPATSANA AGAAAIVGAL AGIPGGPLGV VVGAVSAGLT TAIGSTVGSG SASSSAGGGS

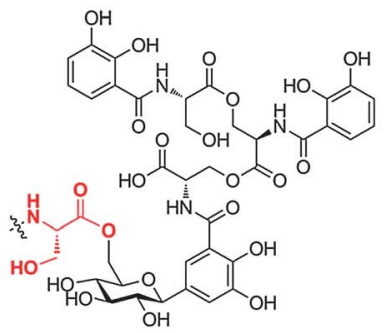

Fig. 16 Structures of the post-translationally modified microcins. A. Structures of several class I microcins. Both the linear sequence and three dimensional structure of microcin J25 is shown (PDB 1Q71). Microcin B17, another class I microcin, is shown in Fig. 9. B. Class II microcins. The post-translational modifications of class IIb microcins attach a linear trimer of 2,3-dihydroxybenzoyl Lserine linked via a C-glycosidic linkage to a $\beta$-D-glucose. The glucose is anchored to the carboxylate of the C-terminal serine residues of the peptides (red font) through an ester linkage to 06 .

esterified with 1,3-propanolamine (Fig. 16A). Microcin J25 is a 21-amino acid lasso peptide. ${ }^{273}$ It consists of a macrolactam, which is formed by the N-terminal amino group and the sidechain carboxylate of an aspartate at position 8 , through which the peptide tail is threaded and firmly trapped by steric interactions, thus forming a lasso. Therefore, microcin J25 belongs to both families of microcins from Enterobacteria and lasso peptides, which are mainly synthesized by Actinobacteria (section 10). ${ }^{273-275}$

Class II microcins consist of higher molecular mass peptides in the $5-10 \mathrm{kDa}$ range. This class is further subdivided into two subclasses, IIa and IIb. Class IIa comprises three 
plasmid-encoded peptides, microcins L, V and $\mathrm{N}$ (also named microcin 24) that are not post-translationally modified and that will not be considered here. Class IIb consists of chromosomeencoded linear microcins that carry a C-terminal siderophore (Fig. 16B). ${ }^{276,277}$

\subsection{Biological activities of microcins}

Microcins display potent antibacterial activity against Gramnegative bacteria, and specifically Enterobacteria. They exhibit diverse mechanisms of antibacterial activity and appear attractive models for the design of novel bioactive peptides with anti-infective properties. ${ }^{\mathbf{1 2 , 2 6 9 , 2 7 8}}$ Microcins have receptor-mediated mechanisms of antibacterial activity that improve their uptake in sensitive bacteria. Therefore, they have a narrow spectrum of activity and minimal inhibitory concentrations in the nanomolar range. Microcins hijack receptors involved in the uptake of essential nutrients, and many parasitize iron siderophore receptors or porins. Those using siderophore receptors are also dependent on the TonB-ExbB-ExbD inner-membrane protein complex, which ensures energy transduction to the outer membrane and its receptors, using the proton-motive force from the cytoplasmic membrane. Microcin J25 uses the receptor to ferrichrome (FhuA), microcins B17 and $\mathrm{C}$ use the porin OmpF, and microcins $\mathrm{E} 492$ and $\mathrm{M}$ use the receptors to catechol siderophores (FepA, Cir and Fiu). After crossing the outer membrane, microcins often require an inner membrane protein to exert their toxic activity, such as SbmA for microcins B17 and J25 or components of the mannose permease for microcin E492. Others require an ABC-transporter, such as YejABEF for microcin C. Many microcins inhibit vital bacterial enzymes, such as DNA gyrase (microcin B17) or RNA polymerase (microcin J25). The latter blocks transcription by binding in the secondary channel that directs NTP substrates toward the RNA polymerase active site, resulting in its obstruction in a cork-inthe-bottle fashion.

A two-step processing procedure inside target bacteria is required to activate microcin $\mathrm{C}$. First peptide deformylase removes the N-terminal formyl group, and then several broadspecificity aminopeptidases target the ultimate peptide bond Ala6-Asp7 and cleave off the N-terminal peptide (Fig. 16A). The resulting molecule is a mimic of aspartyl adenylate, which inhibits aspartyl tRNA synthetase and therefore blocks protein synthesis at the translation step. To exert its bactericidal activity, the class IIb microcin E492 forms channels in the inner membrane.

With the exception of the lasso structure of microcin J25 (see section 10), three-dimensional structures of microcins are not available. ${ }^{273,279-281}$ However, in the absence of precise threedimensional structures, structure-activity relationships of class I microcins have been undertaken. A chemical strategy was developed to prepare a series of microcin $\mathrm{C}$ analogs, which retained the Trojan horse mechanism of antibacterial activity of the natural microcin, while targeting different aminoacyl-tRNA synthetases specified by the last amino acid that is linked to the adenosine. ${ }^{282}$ In the case of microcin B17, point mutations indicate that the number and position of the thiazole and oxazole rings in the microcin structure are essential, and that the C-terminal bis-heterocycle is critical. ${ }^{51}$

\subsection{Microcin biosynthesis}

All microcins except microcin $\mathrm{C}$, which does not possess a leader peptide and is secreted without cleavage of a precursor, are translated from their respective mRNA as precursor peptides consisting of an N-terminal leader peptide and a $\mathrm{C}$ terminal core peptide, which undergoes post-translational modifications prior to becoming the mature active microcin. ${ }^{\mathbf{1 2}}$ Class II microcins result from maturation of large precursors carrying small conserved leader peptides (15-19 amino acids). Class I microcins have longer leaders of 19-37 amino acids that (at present) do not display common features, neither in length nor in amino acid sequences. Removal of the microcin leader peptides most often occurs at a specific cleavage site, the typical double-glycine motif or the glycine-alanine motif found in proteins exported through ABC (ATP-binding cassette) transporters. ${ }^{283}$ The roles of the leader peptides have not been fully clarified to date, but they could contribute to maturation, secretion, protection of the precursor against degradation, or to keeping the precursor inactive inside the host cells during biosynthesis. ${ }^{16}$

The biosyntheses of microcin B17 and J25 have been reconstituted in vitro. ${ }^{\mathbf{5 1 , 2 8 4 , 2 8 5}}$ Mature microcin B17 was obtained from the precursor McbA in the presence of the modification enzymes McbB, McbC, and McbD that form the microcin B17 synthetase complex. ${ }^{51}$ We note that the original gene designations for the microcin B17 gene cluster were alphabetical, ${ }^{286}$ and were different from that recommended in section 5 for LAPs. In this early designation McbB corresponds to the "C" protein of LAPs and McbC was the flavin dependent dehydrogenase (designated B in other LAPs). ${ }^{51,52}$ The oxazole and thiazole heterocycles result from a series of cyclization, dehydration, and dehydrogenation reactions as described in section 5. During the modification process, the McbBCD complex recognizes the leader peptide that has a helical structure. ${ }^{58}$ Upon removal of the N-terminal 26-amino acid leader peptide, presumably by TldD/TldE, ${ }^{287}$ mature microcin B17 is exported from the cell by the dedicated $\mathrm{ABC}$ transporter McbEF.

The maturation process of microcin $\mathrm{C}$ requires five steps including three modifications of the precursor MccA and two cleavage steps that are accomplished in the target cells: ${ }^{278,288}$ (i) conversion of Asn7 to a derivatized isoAsn7, (ii) formation of an $\mathrm{N}-\mathrm{P}$ bond between the amide group of isoAsn7 and the $\alpha$ phosphate of ATP catalyzed by MccB, affording the peptidylAMP linkage, (iii) aminopropylation of the phosphoramidate using two enzymes encoded by $m c c D$ and $m c c E$, (iv) deformylation, and (v) proteolysis at the Ala6-Asp7 peptide bond (see section 9.1).

The modification anchored at the $C$-terminus of microcin E492 is a $C$-glucosylated linear trimer of 2,3-dihydroxybenzoyl-Lserine (DHBS), which itself results from linearization of the cyclic catechol-type siderophore enterobactin. ${ }^{289-291}$ Enterobactin and salmochelin, its glycosylated counterpart, are first made by nonribosomal peptide synthetases. Modification of the 
84-amino acid ribosomally produced peptide at its C-terminal serine with the sidereophore requires four genes mceCDIJ. MceC and MceD ensure enterobactin glucosylation and linearization, while MceI and MceJ are responsible for the formation of the ester linkage between the glucosylated enterobactin and the Cterminal serine of the microcin (Fig. 16B). The biosynthesis of the lasso peptide microcin J25 is discussed in section 10.2.

\subsection{Specific recommendations for the microcin family}

At present, the precursor peptide and common biosynthetic genes encoding proteins involved in export, immunity and modification enzymes are not always given the same gene name (e.g. mcxA for the precursor peptides to the microcins). Given the relatively small number of microcins, we recommend that a common designation of biosynthetic genes will be systematically implemented, including using the nomenclature systems implemented for other RiPPs, such as LAPs and lasso peptides.

\section{Lasso peptides}

Lasso peptides form a growing class of bioactive bacterial peptides that are characterized by a specific knotted structure, the lasso fold. ${ }^{292}$ The producing bacteria are most often Actinobacteria (Streptomyces, Rhodococcus), but some are Proteobacteria (Escherichia, Burkholderia). ${ }^{273-275}$ They consist of 16-21 residues, with an $\mathrm{N}$-terminal macrolactam, resulting from the condensation of the $\mathrm{N}$-terminal amino group with a side-chain carboxylate of a glutamate or aspartate at position 8 or 9. Lactam formation irreversibly traps the C-terminal tail within the macrocycle. The net result is a highly compact and stable structure that to date has proven inaccessible by peptide synthesis. Lasso peptides are remarkably resistant to proteases and denaturing agents, and this high stability and their biological activities have attracted much interest. Indeed, the known lasso peptides inhibit enzymes or antagonize receptors, which confer antimicrobial properties on some of them. ${ }^{273}$ Furthermore, the lasso structure has recently been shown to be an efficient scaffold for epitope grafting, ${ }^{\mathbf{4}}$ demonstrating the capacity to give novel biological functions to the lasso fold.

Lasso peptides are classified into three classes (Fig. 17A). Members of the first class contain two disulfide bonds and the first residue is a cysteine. This class contains four representatives: siamycin I (also called MS-271), ${ }^{293,294}$ siamycin II and RP$71955,{ }^{295,296}$ and SSV-2083. ${ }^{6}$ Class II lasso peptides do not contain disulfides and the first residue is a glycine. This class contains eight representatives: RES-701, ${ }^{297-300}$ propeptin, ${ }^{301,302}$ anantin, ${ }^{303,304}$ lariatins $\mathrm{A}$ and $\mathrm{B},{ }^{305}$ microcin $\mathrm{J} 25,{ }^{306,307}$ capistruin, ${ }^{308,309}$ and SRO15-2005. ${ }^{6}$ The third class, which was recently discovered and encompasses a single peptide, BI$32169,{ }^{310}$ is characterised by the presence of a single disulfide bond and a glycine at the $\mathrm{N}$-terminus. In all of the lasso peptides identified to date, the residue providing the side-chain carboxylate implicated in the macrolactam is a Glu at position 8 or an Asp at position 9 (or position 8 in one example), pointing to a stringent role of the size of the macrolactam in maintaining the lasso fold. A recent genome mining study has identified 76
A
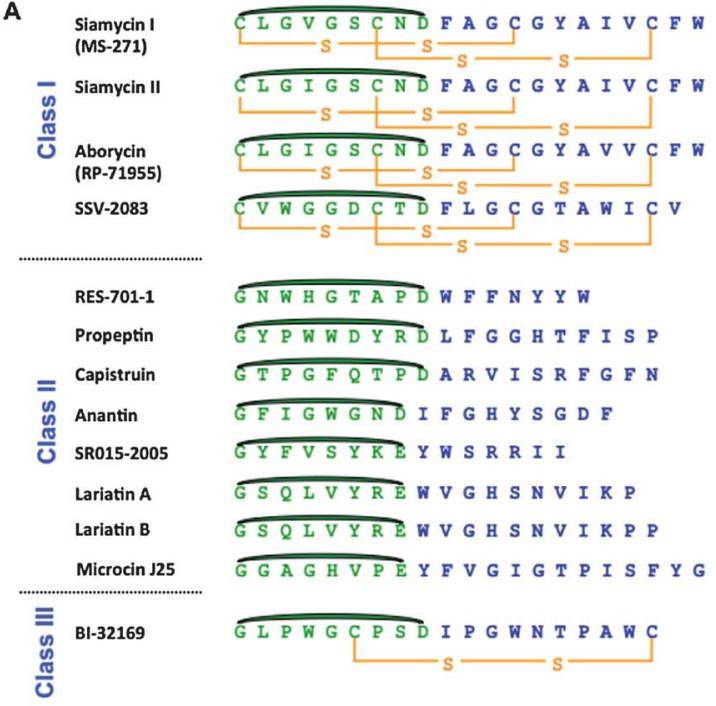

$\widetilde{G L W G C P S D}$ I P G WN TPA W C

B
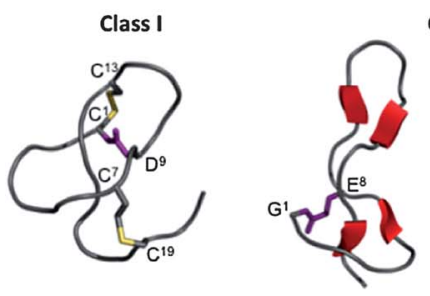

Class II

Class III

Siamycins

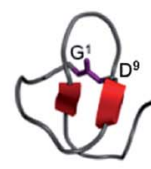

Capistruin

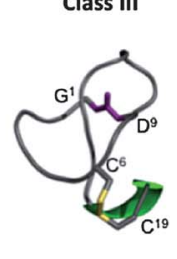

BI-32169

Fig. 17 Structural features of lasso peptides. A) Sequences of lasso peptides of the three classes showing the residues involved in the macrolactam in green and those forming the tail in blue. The isopeptide linkage between the $\mathrm{N}$-terminal amino group and the side chain carboxylates of Asp and Glu are shown in dark green. B) Three-dimensional structures of lasso peptides of the three classes; the structures of the class II microcin $J 25$ and capistruin exhibit the respective sizes of the loop and tail above and below the ring.

potential lasso peptide producers spanning nine bacterial phyla and an archaeal phylum. ${ }^{311}$ One gene cluster from the freshwater bacterium Asticcacaulis excentricus was heterologously expressed in E. coli leading to the production of the largest lasso peptide to date, astexin-1. ${ }^{311}$ It consists of 23 amino acids and unlike most other lasso peptides is highly polar.

Microcin J25 produced by $E$. coli AY25 is the archetype of lasso peptides and the most extensively studied. Its lasso structure of type II results from linkage of the amino group of Gly1 to the side-chain carboxylate of Glu8 to give the N-terminal macrolactam; the threaded 13-residue C-terminal tail (Fig. 17A) is maintained irreversibly in the ring by the bulky side-chains of two aromatic residues, Phe19 and Tyr20, that straddle the ring and prevent the tail from escaping. Its three-dimensional structure contains two small anti-parallel $\beta$-sheets. ${ }^{279-281}$ One involves residues 6-7 located in the ring and residues 18-19 of the threading C-terminal tail. The other is located in the loop region of the lasso structure and comprises residues 10-11 and 15-16 that form a hairpin-like structure including a $\beta$-turn that involves residues 11-14 (Fig. 17B).

Capistruin is a 19-residue peptide that is produced by Burkholderia thailandensis E264 and contains a 9-residue ring 
resulting from a Gly1-Asp9 isopeptide bond. ${ }^{308}$ Six amino acids out of the 10-residue C-terminal segment pass through the ring, forming quite a long tail, which is trapped in the ring by the side-chains of Arg11 and Arg15. Similar to microcin J25, its three-dimensional structure contains a small antiparallel $\beta$ sheet involving residues 7-8 of the ring and residues 13-14 of the threading C-terminal tail that are connected by a $\beta$-turn involving residues 9-12. Having a shorter and tighter loop, capistruin lacks the second $\beta$-sheet present in the loop region of microcin J25.

\subsection{Biological activities of lasso peptides}

Lasso peptides are usually enzyme inhibitors or receptor antagonists. These properties confer some of them with antibacterial activity, which is directed against either Gram-negative bacteria or Gram-positive bacteria. The spectrum of activity is generally limited to bacteria phylogenetically related to the producing bacteria, helping them to colonize a given biotope. In class I, siamycin I (MS-271) is an inhibitor of the myosin light chain kinase and an anti-HIV agent, ${ }^{293,294}$ and siamycin II and RP-71955 are anti-HIV agents. ${ }^{\mathbf{2 9 3 , 2 9 4}}$ In the class II lasso peptides, RES-701 peptides are antagonists of the type $\mathrm{B}$ receptor of endothelins, ${ }^{297-300}$ propeptin is a prolyl endopeptidase inhibitor, ${ }^{301,302}$ anantin is an antagonist of the atrial natriuretic factor, ${ }^{303,304}$ lariatins A and B are anti-mycobacterial agents, ${ }^{305}$ and microcins $\mathrm{J}^{2} 5^{\mathbf{3 0 6 , 3 0 7}}$ and capistruin ${ }^{308,309}$ are RNA polymerase inhibitors and antibacterial agents. The single class III lasso peptide BI-32169 is an antagonist of the glucagon receptor. ${ }^{310}$

\subsection{Lasso peptide biosynthesis}

Microcin J25 and capistruin are the two lasso peptides that have been the most extensively studied with regards to their biosynthetic pathways. Both microcin J25 and capistruin arise from four-gene clusters ( $m c j A B C D$ and $c a p A B C D$ ) in E. coli and $B$. thailandensis, respectively. Microcin J25 has been the model for the study of the biosynthesis of lasso peptides. Its production requires $\mathrm{McjB}$ and $\mathrm{McjC}$ to control the acquisition of the lasso fold from the 58 -amino acid linear precursor. ${ }^{284}$ On the basis of sequence comparisons, ${ }^{269,284} \mathrm{McjB}$ is believed to be an ATPdependent cysteine protease that cleaves off the leader peptide. McjC has homology with Asn synthetase $B,,^{284,285}$ and is hypothesized to form the isopeptide bond between the glycine at position 1 generated upon proteolytic cleavage of the leader and the Glu8 side-chain carboxylate, which is activated through an acyl-AMP intermediate. McjB and McjC are two interdependent enzymes that mutually need the physical presence of their partner to carry out their enzymatic reactions. The distinct functions of $\mathrm{McjB}$ and $\mathrm{McjC}$ were recently demonstrated by in vivo mutagenesis studies of putative active site residues, ${ }^{312}$ and in vitro studies using recombinant precursor and maturation enzymes. ${ }^{313}$ McjC was confirmed to be a lactam synthetase and McjB was shown to be a novel cysteine protease that requires ATP hydrolysis for proteolytic activity. ${ }^{313}$ In view of the prefolding step required for obtaining the lasso fold, the ATPdependent character of McjB could be related to a chaperone function. Interestingly, only the $\mathrm{N}$-terminal eight residues of the leader peptide are required for recognition of the precursor McjA by the maturation machinery. ${ }^{68}$ In particular, the Thr residue in the penultimate position of the leader peptide is proposed to be a recognition element for the maturation enzymes. ${ }^{314}$ The final product is believed to be transported from the cytoplasm by an $\mathrm{ABC}$ transporter. ${ }^{284,315}$ In contrast to microcin J25 and capistruin, which are encoded by four-gene clusters, lariatins A and B, produced by Rhodococcus jostii, result from a five-gene cluster (larABCDE). ${ }^{316}$ It is proposed that processing of the precursor LarA by LarB, LarC and LarD leads to the mature lasso peptide, which is then exported by LarF. ${ }^{316}$ Taking into account the sequence similarities between the larB and $\operatorname{larD}$ gene products and the modification enzymes identified for microcin J25 and capistruin, it is suggested that LarC, the function of which remains unclear, is a third enzyme essential for lariatin biosynthesis that would be specific to Gram-positive bacteria.

Two systematic structure-activity relationship studies of microcin J25 have been performed, paving the way for future studies deciphering the roles of specific amino acids in the lasso structure and antibacterial activity. ${ }^{31,39}$ The most striking conclusion of these studies was that production and activity of the lasso peptide were tolerant to amino acid substitutions at most of the positions in the 21 amino acid core sequence. Another recent study demonstrated that the C-terminal residue, the bulky residue below the ring, and the size of the ring are critical for generating the lasso structure. ${ }^{317}$ Interestingly, some amino acid substitutions afforded branched-cyclic peptides instead of lasso peptides that were devoid of antibacterial activity. Moreover, the tail portion below the ring and the $\mathrm{C}$ terminal glycine appeared critical for the antibacterial activity but could be modified without affecting lasso formation.

Using a heterologous capistruin production system in $E$. coli, a series of mutants of the precursor protein CapA have been generated, showing that only four residues of the lasso sequence are critical for maturation of the linear precursor into the mature lasso structure. ${ }^{41}$ Thus, the biosynthetic machineries of both microcin J25 and capistruin systems have low substrate specificity, which is a promising feature towards lasso peptide engineering.

\subsection{Specific recommendations for the lasso peptide family}

Similar to microcins, a common gene nomenclature should be adopted for lasso peptides in which the precursor and common biosynthetic genes are always given the same gene name: " $A$ " for the genes encoding the precursor peptides, " $B$ " for genes encoding the transglutaminase/cysteine protease homologs, " $C$ " for genes coding for the Asn synthetase homologs, and " $D$ " for genes encoding $\mathrm{ABC}$ transporters.

\section{Microviridins}

Microviridins represent a family of cyclic $N$-acetylated trideca and tetradecapeptides that contain intramolecular $\omega$-ester and/ or $\omega$-amide bonds. Like the lasso peptides, most microviridins contain lactams, but unlike the lasso peptides, these structures 
are not formed from the N-terminal amine but rather between $\omega$-carboxy groups of glutamate or aspartate and the $\varepsilon$-amino group of lysine. ${ }^{318}$ Furthermore, microviridins also contain lactones generated by esterification of the $\omega$-carboxy groups of glutamate or aspartate with the hydroxyl groups of serine and threonine. Lactone and lactam formation results in an unparalleled tricyclic architecture (Fig. 18). The crosslinking amino acids are part of a conserved TXKXPSDX(E/D)(D/E) central motif, whereas the $\mathrm{N}$ - and the C-termini of microviridin core peptides are highly variable. ${ }^{319}$ The related bicyclic dodecapeptide marinostatin (Fig. 18) contains only $\omega$-ester bonds and a slightly altered microviridin-type central motif (TXRXPSDXDE). ${ }^{\mathbf{3 2 0}}$

Microviridins have so far only been detected in bloomforming freshwater cyanobacteria of the genera Microcystis and Planktothrix. The prototype of the family, microviridin A, was described for Microcystis viridis strain NIES 102 in 1990..18 Eleven further structures of related cyanobacterial metabolites,

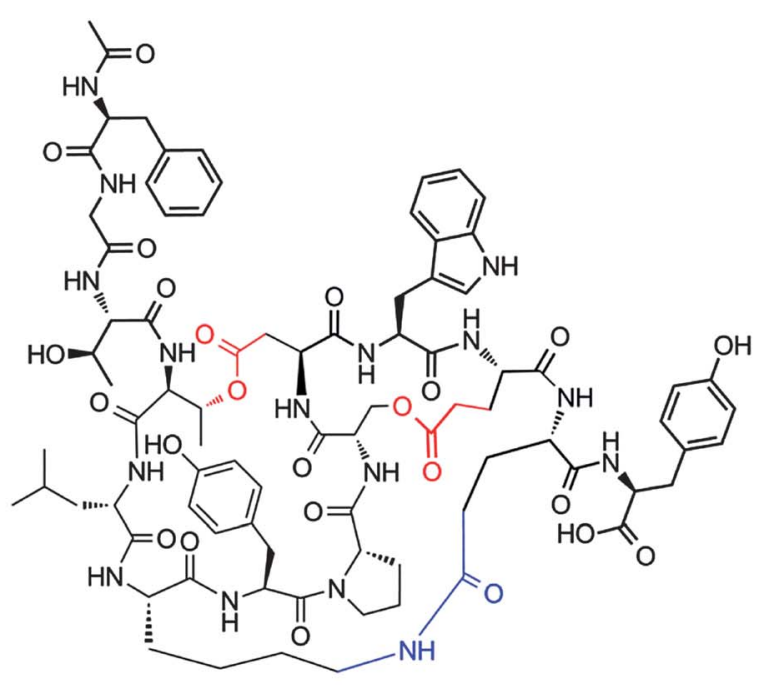

\section{Microviridin B}

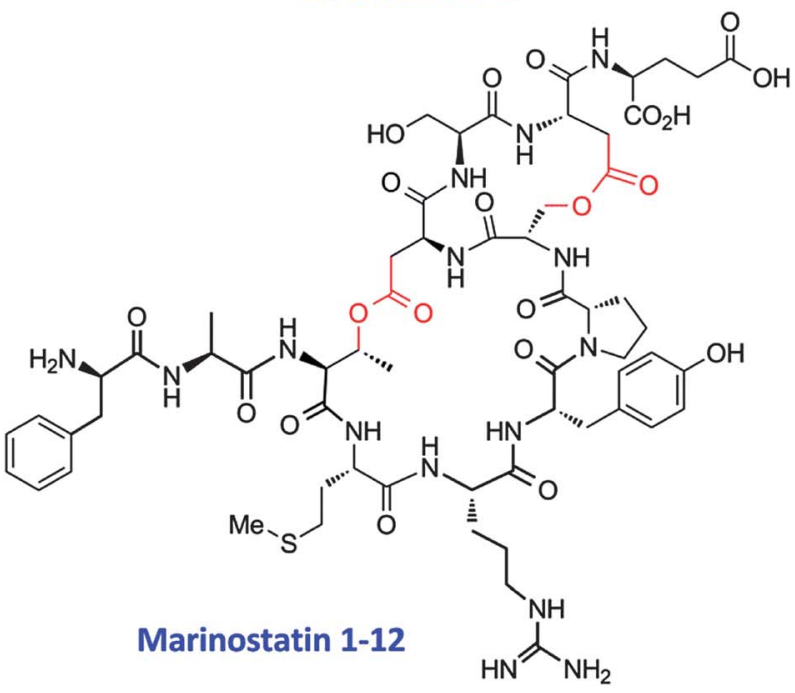

Fig. 18 Structures of microviridin B from $M$. aeruginosa NIES298 and marinostatin 1-12 from Alteromonas sp. B-10-31.322,325 Amino acid side chains forming ester and amide bonds are highlighted in red and blue, respectively. microviridin B-L, were elucidated in the past twenty years from laboratory strains and field samples..$^{321-324}$ Bioinformatic analysis of complete microbial genomes, however, suggests a more widespread potential for microviridin biosynthesis than expected. Putative microviridin precursor genes were detected in a large number of cyanobacterial genomes, including strains of Cyanothece, Anabaena, Nodularia and Nostoc. ${ }^{323}$ In addition, the presence of related microviridin-type gene clusters in genomes of the sphingobacterium Microscilla marina ATCC 23134 and the myxobacterium Sorangium cellulosum Soce56 suggests an occurrence of microviridins beyond the cyanobacterial phylum, ${ }^{323}$ and the related bicyclic peptide marinostatin has been detected in the marine proteobacterial strain Alteromonas sp. B-10-31. . $^{320}$

\subsection{Biological activities of microviridins}

Microviridins were initially discovered through a bioactivityguided screening program for inhibitors of serine type proteases in cyanobacteria. ${ }^{318}$ The different variants show distinct protease inhibition profiles with specificities for elastase,

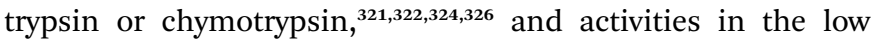
nanomolar range. ${ }^{322,326}$ For example, microviridin B (Fig. 18) specifically inhibits elastase and has potential in the treatment of lung emphysema, ${ }^{322}$ and microviridin J inhibits a trypsin-type enzyme from Daphnia and disrupts the molting process of the animals. ${ }^{326}$ Microviridins may therefore play an important role in the trophic networks of freshwater lakes. Similarily, the bicyclic marinostatin (Fig. 18) has inhibitory activity against the protease subtilisin. ${ }^{320}$

\subsection{Biosynthesis of microviridins}

Microviridin biosynthesis has been partially characterized in vivo and in vitro for the two cyanobacterial genera Microcystis and Planktothrix. ${ }^{323,327}$ The corresponding gene clusters have been designated $m d n A-E$ and $m v d A-E$, respectively. ${ }^{323,327}$ The precursor peptides MdnA and MvdE contain a strictly conserved PFFARFL motif in their leader peptide that is essential for posttranslational modification reactions. ${ }^{319}$ The lactone and lactam structures are introduced by two novel types of ATP grasp ligases, MdnC and MdnB, and MvdD and MvdC, respectively. ${ }^{323,327}$ The four enzymes show close similarity to each other and belong to the RimK family of enzymes that catalyze crosslinks of carboxyl groups with amine or thiol groups. ${ }^{328}$ In vitro characterization revealed the activity of $\mathrm{MvdD}$ as the $\omega$-ester ligase and MvdC as the $\omega$-amide ligase. ${ }^{323}$ Further analysis of MvdD and mutational studies of the precursor peptide MvdE in Planktothrix suggested a strict order for the formation of the crosslinks, with the larger lactone forming first and the smaller lactone forming second. ${ }^{329}$ The size of both rings appears to be inflexible. ${ }^{329}$ Lactonization is then followed by lactam formation via MvdC. ${ }^{323}$ The cluster further encodes a GNAT-type $N$-acetyl transferase, designated $\mathrm{MdnD}$ and $\mathrm{MvdB}$ in Microcystis and Planktothrix, respectively. ${ }^{323,327} \mathrm{MvdB}$ catalyzes the $N$-acetylation of the N-terminal amino acid of the lactonized and lactamized premature peptide. ${ }^{323}$ The microviridin gene cluster does not encode a protein with protease signatures that could be 
responsible for the cleavage of the leader peptide. However, the associated $\mathrm{ABC}$ transporter $\mathrm{MdnE}$ is crucial for correct processing in vivo and may play a scaffolding role thereby guaranteeing correct processing. ${ }^{319}$ Interestingly, the putative gene cluster in Anabaena PCC7120 encodes a precursor peptide that contains multiple putative core peptides, ${ }^{327}$ similarly to the multiple RiPPs generated from a single peptide that has been found for cyanobactins and cyclotides (sections 6 and 15).

\subsection{Specific recommendations for the microviridin family}

The characteristic lactone and lactam structures are the signature motifs that define this group. Moreover, the three peptide modifying enzymes do not show close similarity to enzymes of other PRPS classes. The two enzymes catalyzing macrocyclization, MdnC/MvdD and MdnB/MvdC, can thus be taken as enzymatic basis for the classification of the microviridin group of peptides. Marinostatin and the corresponding biosynthesis gene cluster do not meet all criteria. However, as the two lactones and the ATP grasp ligase encoded by the marinostatin gene cluster resemble characteristic features of the microviridin class, marinostatins should be considered as a specific subgroup of microviridins. Two different gene nomenclatures $(m d n, m v d)$ have been assigned to the related gene clusters in the two cyanobacterial genera. ${ }^{323,327}$ As both in vivo and in vitro characterization provided important insights into microviridin biosynthesis, standardization of this historical nomenclature is not possible without omitting important results. Future investigations into microviridins, however, should follow at least one of the existing gene designations.

\section{Sactipeptides: peptides with cysteine sulfur to $\alpha$-carbon crosslinks}

This section focuses on a small but growing class of ribosomally synthesized peptides that have undergone post-translational modification to introduce intramolecular linkages between cysteine sulfur and the $\alpha$-carbon of another residue. Although some diketopiperazines (cyclodimers of amino acids) such as gliotoxin and aranotin have long been known to have sulfur to $\alpha$-carbon linkages, ${ }^{330}$ those compounds are made by non-ribosomal peptide synthetase systems. At least for the case of gliotoxin, the sulfur is introduced by nucleophilic attack of glutathione on a diketopiperazine $N$-acyl imine. ${ }^{331}$ The first report of a ribosomally-made peptide with a sulfur to $\alpha$-carbon linkage was the structural elucidation in 2003 of subtilosin A. ${ }^{332}$ In the meantime at least six such structures have been reported from Bacillus species. These include a naturally occurring hemolytic subtilosin A mutant (T6I) ${ }^{333}$ from B. subtilis, the anticlostridial Trn- $\alpha$ and Trn- $\beta$ from $B$. thuringiensis (together forming the two-component system thuricin CD), ${ }^{334}$ thurincin $\mathrm{H}$ from another strain of $B$. thuringiensis, ${ }^{335}$ and the cannibalistic sporulation killing factor (SKF) from B. subtilis (Fig. 19). ${ }^{336}$ The term sactibiotic (sulfur to alpha-carbon antibiotic) has been suggested to describe peptides in this group. ${ }^{337}$ The more general term sactipeptide may be appropriate as some compounds with such a structural feature but lacking antimicrobial activity may be discovered in the future. The sulfur to $\alpha$ carbon linkage is also found in cyclothiazomycin, a thiopeptide from Streptomyces hygroscopicus that has a C-terminal cysteine sulfur to $\alpha$-carbon link with an alanine. ${ }^{338-340}$

All sactipeptides are relatively hydrophobic, and in the cases examined by NMR spectroscopy, have a 3D solution structure that resembles a hairpin with hydrophobic residues pointing to the exterior (Fig. 20). These include subtilosin $A,{ }^{332,341}$ Trn- $\alpha$ and
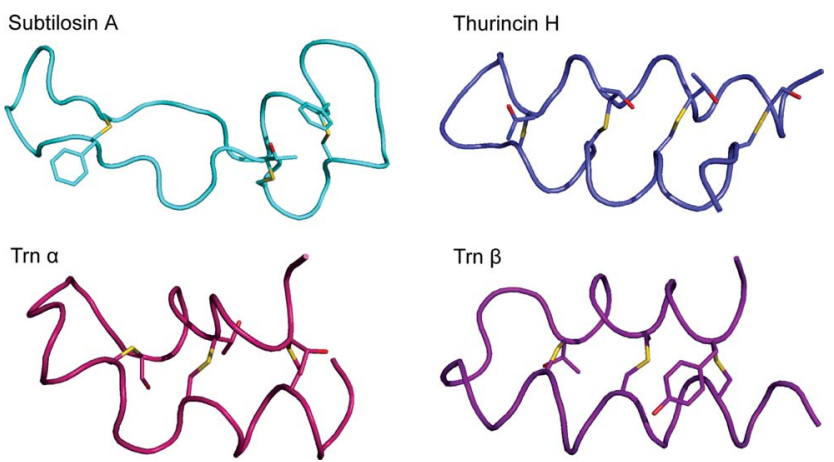

Fig. 20 Three dimensional solution structures of sactipeptides. Subtilosin A has $\mathrm{S}$ to $\mathrm{C} \alpha$ crosslinks between C4 and F31, C7 and T28, and C13 and F22. Thurincin $\mathrm{H}$ has such links between C4 and S28, C7 and T25, C10 and T22, and C13 and N19. Trn- $\alpha$ has links between C5 and T28, C9 and T25, and C13 and S21. Trn- $\beta$ has links between $C 5$ and $Y 28, C 9$ and $A 25$, and $C 13$ and T21. SKF (not shown, 3D structure not reported) has a single $S$ to $C \alpha$ crosslink between $C 4$ and $M 12$. It also has a disulfide bond between $\mathrm{C} 1$ and $\mathrm{C} 16$.

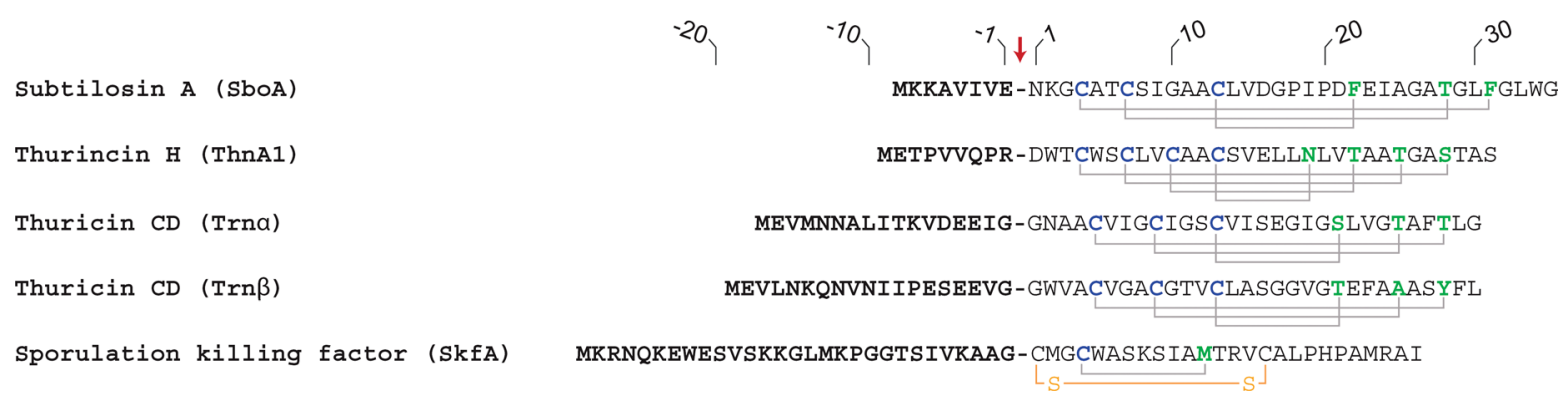

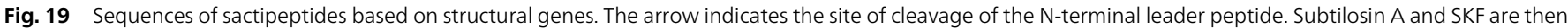

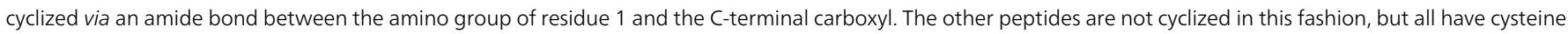
residues linked to $\alpha$-carbons. 
Trn- $\beta,^{342}$ and thurincin $\mathrm{H}^{335}$ In subtilosin $\mathrm{A}$ and its T6I variant, ${ }^{333}$ as well as in $\mathrm{SKF},{ }^{336}$ the $\mathrm{C}$ - and $\mathrm{N}$-termini at the ends of the hairpin are cyclized to form an amide bond. Both circular and open hairpin structures are conformationally restricted by the crosslinks of cysteine sulfur to the $\alpha$-carbon of residues on the opposite side of the structure (Fig. 20). This unusual linkage is relatively stable under acidic conditions, but treatment with base and reducing agent rapidly cleaves it to form a free cysteine and a reactive $N$-acyl imine intermediate ${ }^{341}$ that can tautomerize to a more stable enamine form. An analogous process resulting in a related thiol elimination product occurs during mass spectrometric analysis, and can thereby give the initial impression that the cysteine residues are not modified and that the residues originally linked at the $\alpha$-carbon are dehydroamino acids. $^{334-336}$

Most of the compounds isolated thus far having this modification show some antimicrobial activity, but the detailed mechanisms are not well understood. The high potency, and in some cases narrow specificity (e.g. Trn- $\alpha$ and Trn- $\beta),{ }^{343}$ of antimicrobial action suggests that many of these molecules require recognition of a receptor molecule in the cell membrane of target organisms. Thus far only the interaction of subtilosin A with lipid bilayers has been examined in detail. ${ }^{344,345}$ Subtilosin A adopts a partially buried orientation in such lipid bilayers. Interestingly, subtilosin A shows spermicidal activity although it is not haemolytic at low concentrations. ${ }^{346}$ However, replacement of its threonine- 6 with isoleucine gives a variant that is potently haemolytic. ${ }^{333}$ As expected for a bacteriocin produced by a Gram-positive organism (B. subtilis), subtilosin A displays good activity against other Gram-positive species. Surprisingly, this effect is inhibited by EDTA through an unknown mechanism. ${ }^{347}$ However, at high concentrations in the presence of EDTA, subtilosin A can penetrate the outer membrane of some Gram-negative species to exert an antimicrobial effect.

The key sulfur to $\alpha$-carbon crosslinks in subtilosin were proposed to be generated by a single radical $S$-adenosylmethionine (SAM) enzyme AlbA, ${ }^{348}$ a hypothesis that was recently confirmed by in vitro reconstitution of enzyme activity. ${ }^{349} \mathrm{AlbA}$ was shown to sequentially install the crosslinks of all three cysteines with one threonine and two phenylalanine residues on the linear subtilosin precursor still bearing the leader peptide. $^{349}$ The presence of the leader was essential for activity, and the enzyme distinguished between different residues to which the cysteine is coupled. Similarly, a single gene for a radical SAM enzyme is present in the cluster that is proposed to be responsible for generating all four crosslinks in thurincin $\mathrm{H},{ }^{350}$ and two such genes are found in the thuricin CD cluster, presumably one for crosslinking $\operatorname{Trn}-\alpha$ and the other for modifying Trn- $\beta .^{334}$ The requirement for such radical SAM enzymes has recently been used for genome mining to identify other possible peptides that may have analogous modifications., ${ }^{4,5}$ In addition to their involvement in sactipeptide biosynthesis, radical SAM proteins are also found near peptides that do not contain Cys and that therefore cannot make thioether linkages between Cys sulfurs and alpha carbons. Examples are found in the proteusins and PQQ biosynthetic pathways (sections 4 and 20). Hence, the presence of radical-SAM proteins must be evaluated in the context of the entire gene cluster and/ or structure elucidation of the resulting RiPP before functional roles can be assigned.

\subsection{Specific recommendations for sulfur to $\alpha$-carbon linked peptides}

To broaden the scope with respect to possible future biological activities that may be discovered, these compounds should be called sactipeptides rather than sactibiotics. A common gene nomenclature should be adopted for this group, at least for those cases where the genes are homologous. This nomenclature should be based on the thuricin $\mathrm{CD}$ genes, ${ }^{334}$ using the designation "A" for the precursor peptide, "C" and " $D$ " for the radical-SAM protein(s), and " $F$ " and " $G$ " for ABC-transporters. The designation "P" should be used for currently unidentified proteases that may be encoded in biosynthetic gene clusters that await discovery.

\section{Bacterial head-to-tail cyclized peptides}

Although many peptides are intramolecularly cyclized via amide bonds, this section focuses exclusively on relatively large bacterial peptides (35-70 residues) that are ribosomally synthesized and have a peptide bond between the $\mathrm{C}$ and $\mathrm{N}$ termini. As discussed below, these peptides are distinguished from other head-to-tail cyclized peptides discussed in this review (cyanobactins, amatoxins, orbitides, and cyclotides) by both their size and the biosynthetic machinery that achieves macrocyclization. The most well-studied and famous of these is enterocin AS-48, a potent antimicrobial agent isolated from Enterococcus, ${ }^{351}$ but over the last decade at least nine others have been purified and characterized (Fig. 21). ${ }^{200}$ All are bacteriocins from Gram-positive organisms. They include: butyrivibriocin AR10 (BviA), ${ }^{352}$ gassericin A (reutericin 6) (GaaA), ${ }^{353,354}$ circularin A (CirA), ${ }^{355}$ subtilosin A (SboA), ${ }^{332,341,348,356}$ uberolysin (UblA), ${ }^{357}$ carnocyclin A (CclA), ${ }^{358,359}$ lactocyclicin Q (LycQ), ${ }^{360}$ leucocyclicin $\mathrm{Q}$ (LcyQ), ${ }^{361}$ garvicin ML (GarML) ${ }^{362}$ and the cannibalistic sporulation killing factor (SKF) of Bacillus subtilis. ${ }^{336}$ Recent reviews have appeared on the structures, properties and genetics of production of this group, ${ }^{55,200}$ and more specifically, on enterocin AS-48. ${ }^{351}$

Although most of these peptides show little sequence homology, they tend to be resistant to high temperature $\left(100{ }^{\circ} \mathrm{C}\right)$ treatment, to cleavage by many proteases, and to alterations in pH. They are usually quite hydrophobic and appear to exert their antimicrobial activity by targeting the cell membranes of other bacteria to create pores and cause ion permeability with consequent cell death. They have been classified into three groups. ${ }^{200,359}$ Type $i$ are the AS-48 like peptides that also includes uberolysin, circularin A, lactocyclicin Q, and carnocyclin A. These peptides tend to be cationic at physiological $\mathrm{pH}$ and have relatively high isoelectric points ( $\mathrm{pI}>9$ ). Type ii are the gassericin like peptides that includes butyrivibriocin AR10. These are anionic or neutral at physiological $\mathrm{pH}$ and have lower isoelectric points. Subtilosin A (35 residues) and its variants ${ }^{333}$ comprise an unrelated group that are not only much smaller, 


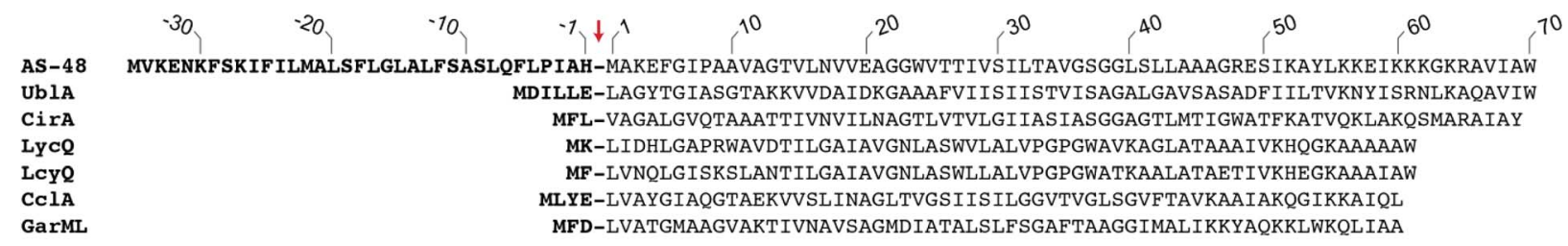

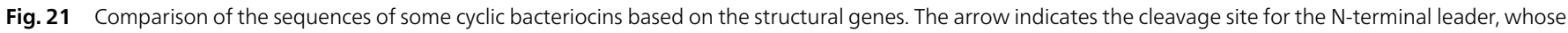
removal is followed by amide bond formation between the amino group of residue 1 and the carboxyl of the C-terminal residue.

but also have three sactipeptide crosslinks as discussed in the previous section. The SKF peptide is also small (26 residues), has one sactipeptide link and one disulfide bridging the ring and is more closely related to subtilosin A than the larger circular peptides. ${ }^{336}$ The genetic determinants of a number of circular bacteriocins have been reported, and they can be located either on the chromosome or on plasmids. The structural genes all encode $\mathrm{N}$-terminal leader peptides that are cleaved during maturation, but the length of these can vary from 2 residues (lactocyclicin Q) to 35 residues (AS-48). A significant number of other genes (up to 10 genes including the structural bacteriocin gene) are often necessary to obtain full production of the cyclic antimicrobial peptide. Many of these are predicted to code for membrane-bound proteins of uncertain function.

The three dimensional structures of enterocin AS-48 (X-ray crystallography), ${ }^{363}$ carnocyclin A (NMR spectroscopy), ${ }^{359}$ and subtilosin A (NMR spectroscopy) $)^{332,341}$ have been reported. Despite low sequence similarity, the three dimensional structures of the large bacteriocins AS-48 (70 residues) and carnocyclin A (60 residues) are remarkably similar and have a saposin fold of 5 and $4 \alpha$ helices, respectively (Fig. 22). Structural predictions based on the sequences of the other large type $i$ and type ii cyclic bacteriocins suggests that they too may have saposin fold arrangements with four-helix bundles capped by either another $\alpha$-helix as in AS-48 or with a less structured loop that replaces it for the shorter peptides of 60 residues. ${ }^{359}$ Despite similarities in three dimensional structures, the biological functions of these circular peptides appear to be quite different. For example, carnocyclin A is monomeric and shows anion binding and membrane transport properties. ${ }^{364}$ In contrast, AS-48 is dimeric and the dynamic reorganization of the interface between the two units is believed to play a key role in membrane pore formation. ${ }^{363}$ Subtilosin A is much smaller (35 residues) and has a quite different structure that does not belong to the saposin fold (see section 12). ${ }^{32,341}$ For all of these cyclic peptides, the N-to-C cyclizations appear to rigidify the structures in such a way that a significant number of hydrophobic residues are displayed to the outside, which is presumably important for their antimicrobial function. However, the detailed mechanisms of action of most of these head-to-tail cyclized peptides are not known.

\subsection{Biosynthesis of bacterial head-to-tail cyclized peptides}

In terms of biosynthesis, the structural genes for these peptides indicate formation of a precursor with an N-terminal leader sequence that can vary greatly in length. The $\mathrm{N}$ terminal leaders for leucocyclicin $\mathrm{Q}$ and lactocyclicin $\mathrm{Q}$ consist of only two residues. ${ }^{\mathbf{3 6 0 , 3 6 1}}$ Circularin A and uberolysin are initially made with $\mathrm{N}$-terminal leaders of three and six residues, respectively. ${ }^{355,357}$ In contrast, enterocin AS-48 has a 35 residue leader. ${ }^{351}$ In all cases discovered thus far, the structural gene shows no additional residues at the C-terminus, unlike the precursors for cyanobactins, amatoxins, cyclotides, and most orbitides (sections 6 and 14-16). This observation suggests that some type of activation of the C-terminal carboxyl group must be accomplished by another enzyme to assist amide bond formation with the amino group at the $\mathrm{N}$ terminus after leader peptide cleavage. However, the identities of such activating enzymes have not been confirmed in the gene clusters for biosynthesis of these circular peptides (Fig. 23). Generally the head-to-tail ligation occurs between two hydrophobic residues, and membrane bound proteins may be involved. When either Met1 or Trp70, which are involved in the head-to-tail cyclization of enterocin AS-48, were substituted with alanine, the amide bond formation still proceeded albeit at lower production levels. ${ }^{365}$ Replacement of the last amino acid, histidine, of the leader peptide with isoleucine blocked formation of AS-48, suggesting that enzymatic recognition of that residue is essential for the leader peptide cleavage reaction. ${ }^{365}$ With the exception of the gene clusters for subtilosin A and SKF, the operons for production of circular peptide bacteriocins generally contain a membrane-bound protein of unknown function referred to as DUF95. ${ }^{55}$ This protein may be involved in the maturation process and/or cyclization.

It is interesting to note that all the gene clusters for the circular bacterial peptides also contain ATP-binding proteins that could potentially activate the $\mathrm{C}$-terminal carboxyl group for cyclization, although they may be responsible for other processes involving export. All the clusters also have a small immunity protein of unknown function that may at least partly protect the producing organism from its own cyclic antimicrobial peptide..$^{55}$

\subsection{Specific recommendations for bacterial N-to-C cyclized peptides}

Historically, a common gene nomenclature has not been adopted for this group (Fig. 23), except for using the designation "A" for the precursor peptide. Since the "B" designation has been mostly, but not uniformly, used for the 
A

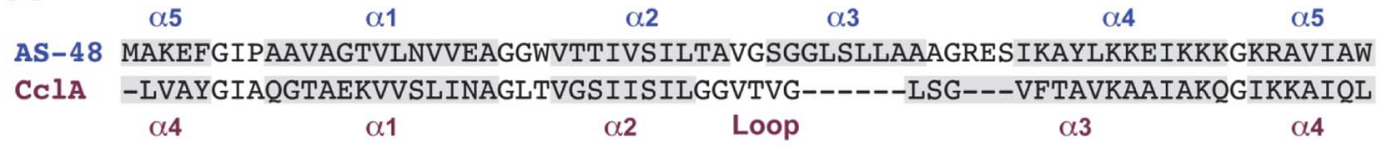

B
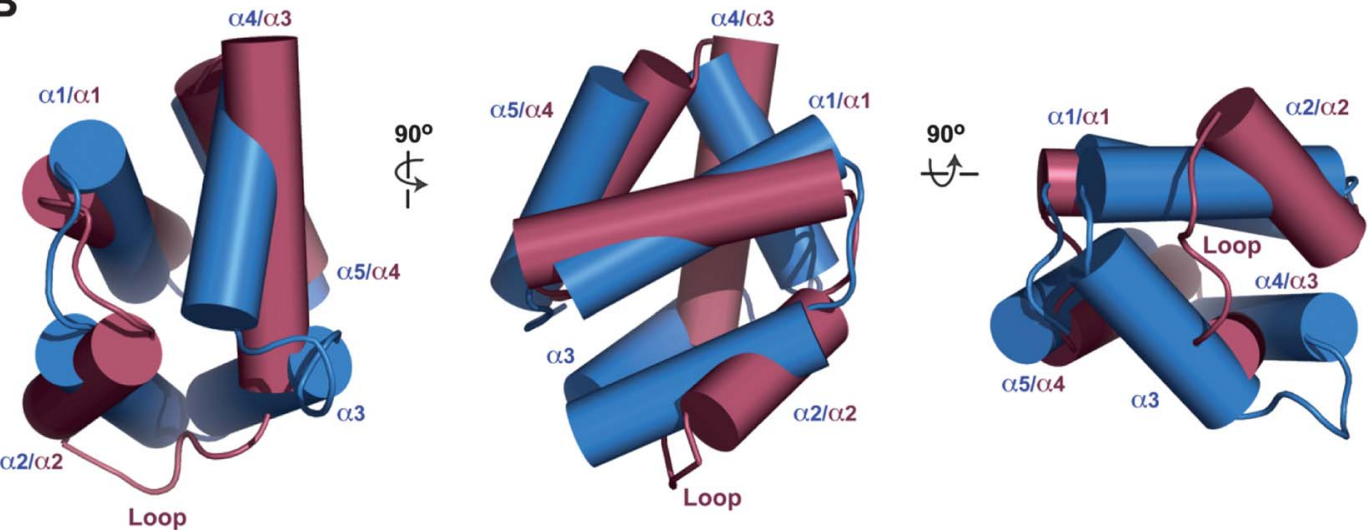

C
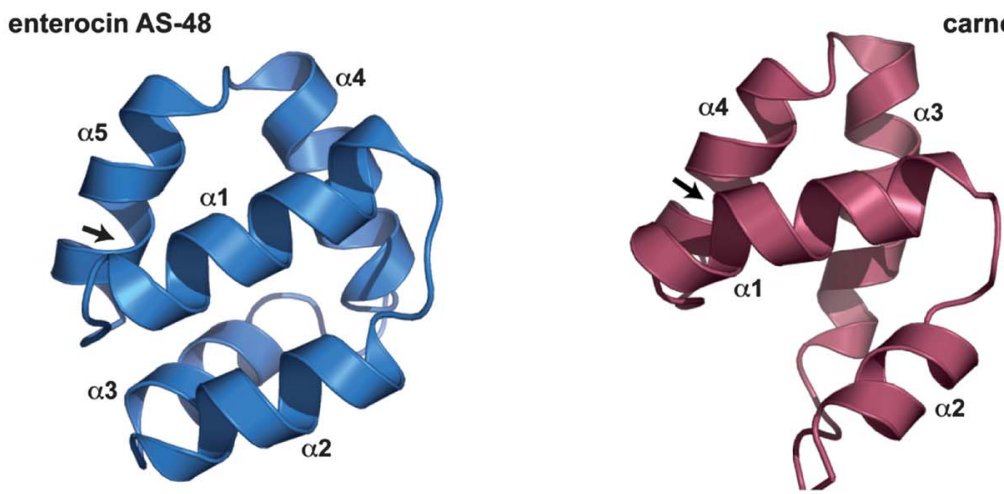

Fig. 22 Comparison of the three dimensional structures of enterocin AS-48 (blue) and carnocyclin A (magenta). (A) Sequence comparison with helices and loop indicated. (B) Overlay of backbones of the two peptides showing the saposin fold and similarities in structure. (C) Ribbon diagrams for the two peptides.

putative membrane protein, we recommend using this designation for new gene clusters that may be discovered in the future.

\section{Amatoxins and phallotoxins}

The amatoxins and phallotoxins are produced by several species of mushrooms in the genera Amanita, Galerina, Lepiota, and Conocybe. ${ }^{54}$ They are N-to-C cyclized peptides of 7 and 8 residues, respectively, that also contain several other post-translational modifications including a tryptathionine formed by crosslinking a Cys to a Trp residue (Fig. 24A). ${ }^{366}$ The tryptathionine in $\alpha$-amanitin is oxidized to the sulfoxide and hydroxylated on the indole in addition to other posttranslational modifications such as a bishydroxylated Ile and cis-4-hydroxyPro. The phallotoxins are structurally similar but have one amino acid less. The amatoxins potently inhibit RNA polymerase $\mathrm{II}^{367}$ whereas the phallotoxins such as phalloidin bind to actin ${ }^{368}$ and have been used to stain the cytoskeleton. The precursor peptides contain a 10-residue leader peptide, a 7- or 8-residue core peptide for phallotoxins and amatoxins, respectively, and a C-terminal recognition sequence (Fig. 24B). ${ }^{22}$ Based on genomic survey sequences and chemical characterization, the core peptide of this family of ribosomal peptides can range in size from 6 to 10 amino acids. Like the cyanobactins and cyclotides discussed elsewhere in this review, the leader and recognition sequences are highly conserved, but the core peptide is more variable. Interestingly, the P1 position for both proteolytic events that excise the core peptide from the precursor peptide is occupied by a proline. The proteins that carry out cyclization, hydroxylation of Leu, Ile and Pro, epimerization (for phallotoxins which contain one D-amino acid), and crosslinking of the Cys and Trp residues are currently still unknown, but a Pro oligopeptidase has been shown to cleave the precursor peptides after the Pro residues in an ordered manner ${ }^{369}$ just as was found for the cyanobactins. $\alpha$-Amanitin is also biosynthesized on ribosomes and initially processed by a Pro oligopeptidase in Galerina marginata, a mushroom not closely related to Amanita. ${ }^{370}$ 
Enterocin AS-48

(Enterococcus faecalis S-48)

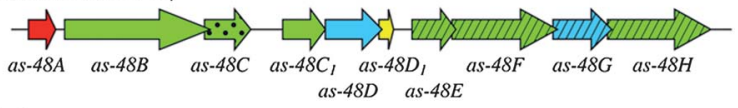

Circularin A

(Clostridium beijerinckii ATCC 25752)

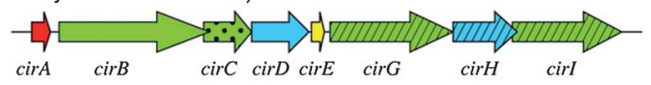

Carnocyclin A

(Carnobacterium maltaromaticum UAL307)

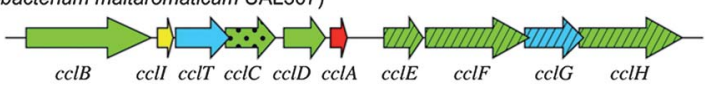

Uberolysin

(Streptococcus uberis 42)

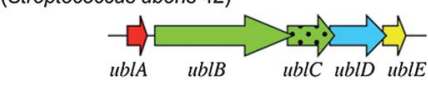

Gassericin A

(Lactobacillus gasseri LA39)

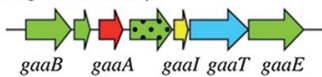

gaaB gaaA gaal gaaT gaaE
gaaC gaaD

Butyrivibriocin AR10

(Butyrivibrio fibrisolvens AR10)
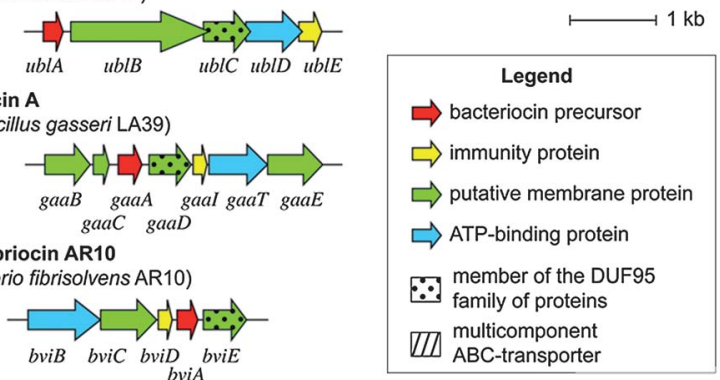

Fig. 23 Comparison of gene clusters for biosynthesis of large ring bacteriocins.

\section{Cyclotides}

The name cyclotides was introduced for ribosomally-synthesized peptides from plants that are characterized by a head-totail cyclic peptide backbone and a cystine knot arrangement of three conserved disulfide bonds. ${ }^{371}$ They were originally discovered in plants of the Rubiaceae (coffee) and Violaceae (violet) families but have since also been found in the Cucurbitaceae (squash) and Fabaceae (legume) families. They are expressed in many plant tissues, including leaves, stems, flowers and roots. Dozens to hundreds of different cyclotides are expressed in an individual plant and there appears to be very little crossover of cyclotides between different plants, i.e., most plants have a unique set of cyclotides.

The prototypic cyclotide, kalata B1, from the African herb Oldenlandia affinis (Rubiaceae) comprises 29 amino acids, including the six conserved Cys residues that form the signature cyclic cystine knot (CCK) motif of the family (Fig. 25). ${ }^{372}$ The backbone segments between successive Cys residues are referred to as loops and the sequence variations of cyclotides occur within these loops. Cyclotides have been classified into two main subfamilies, Möbius or bracelet, based on the presence or absence of a cis X-Pro peptide bond in loop 5 of the sequence. A smaller, third group is referred to as the trypsin inhibitor subfamily. This third subfamily has high sequence homology to some members of the knottin family of proteins from squash plants and its members are also referred to as cyclic knottins. ${ }^{373}$

Thus far more than 200 sequences of cyclotides have been reported and they are documented in a database dedicated to circular proteins called CyBase (www.cybase.org.au). ${ }^{374}$ They range in size from 28-37 amino acids and are typically not highly charged peptides. Aside from their disulfide bonds ${ }^{375}$ and
A

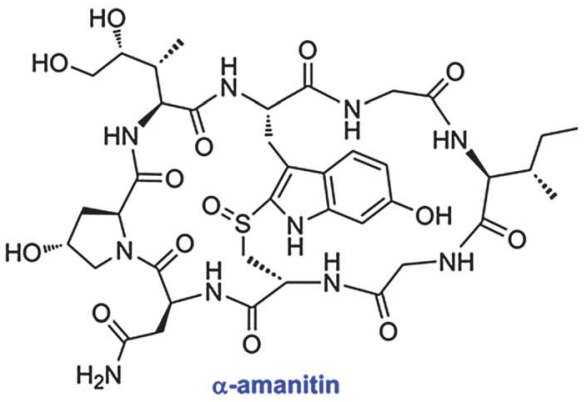

B

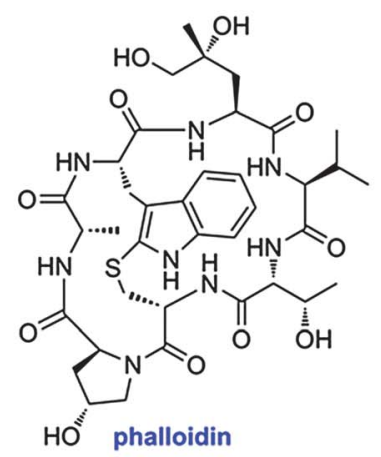

leader peptide

recognition sequence $+1$

$\alpha$-amanitin A8W7M4 MSDINATRLP-IWGIGCNP-CVGDDVTTLLTRGEALC $\beta$-amanitin ABW87785 MSDINATRLP-IWGIGCDP-CIGDDVTILLTRGE--phallacidin ABW87771 MSDINATRLP-AWLVDCP--CVGDDVNRLLTRGESLC phalloidin ABW87787 MSDINATRLP-AWLATCP--CAGDDVNPLLTRGE---

Fig. 24 A. Chemical structures of a representative amatoxin and phallotoxin. B. Alignment of a select number of precursor peptides illustrating the highly conserved leader peptide and recognition sequences in red. The core peptide that is post-translationally modified, excised, and cyclized is shown in blue font. For beta-amanitin and phalloidin, the sequences are from genomic DNA whereas the other two sequences are from cDNA. Because of an intron in the genomic DNA, the last three amino acids of the recognition sequence are not known with certainty for beta-amanitin and phalloidin and are represented with dashes.

head-to-tail cyclized backbone, no other post-translational modifications have been found thus far in cyclotides. Cyclotides appear to be ubiquitous in the Violaceae but are more sparsely distributed in the Rubiaceae, occurring in about $5 \%$ of the hundreds of plants screened thus far. The reports of cyclotides in other plant families are more recent and there is limited information available on the distribution of cyclotides in these families. Nevertheless, it appears that cyclotides are a very large family of plant proteins, potentially numbering in the tens of thousands. ${ }^{376}$

Cyclotides are gene-encoded and are processed from larger precursor peptides. The precursors of Rubiaceae and Violaceae cyclotides are dedicated proteins, whose purpose appears to be only to produce cyclotides. By contrast, cyclotides in Clitoria ternatea, a member of the Fabaceae family, are produced from a chimeric precursor protein that also encodes an albumin, ${ }^{377,378} \mathrm{a}$ situation similar to that recently reported for a small cyclic peptide trypsin inhibitor from sunflower seeds. ${ }^{377}$ Thus, there appear to be multiple types of precursors leading to circular proteins in plants. In the case of Rubiaceae and Violaceae cyclotides, the leader sequence is considered to comprise a 


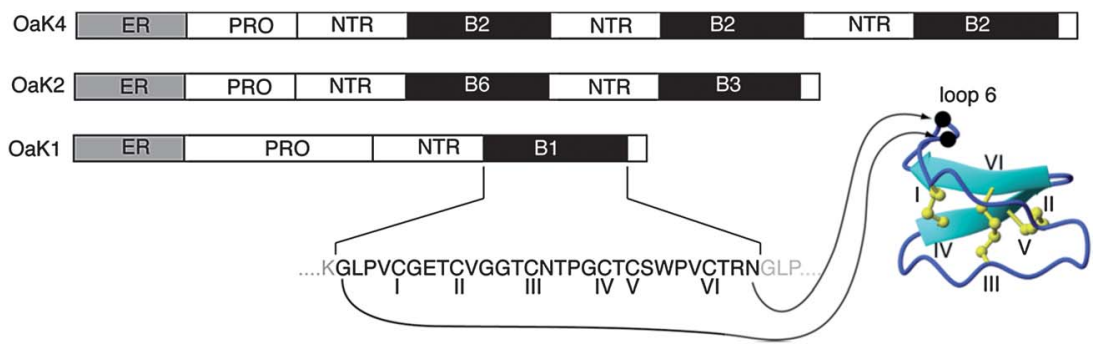

Fig. 25 Structure of kalata B1 and cyclotide precursor proteins. A schematic representation of three precursor proteins from $O$. affinis is shown at the top of the figure. The proteins comprise an endoplasmic reticulum signal sequence labeled ER, a leader sequence (comprising a pro-region and a conserved repeated fragment labeled NTR) and either one or multiple copies of the core peptides. A short hydrophobic C-terminal recognition sequence is present in each precursor. At the bottom of the figure the amino acid sequence of kalata B1 is shown, with the cysteine residues labeled with Roman numerals. The cleavage sites for excision of a core cyclotide domain with an N-terminal Gly and a C-terminal Asn, which are thought to be subsequently linked by an asparaginyl endopeptidase, are indicated by arrows. The location of the ligation point, which forms loop 6 of the mature cyclic peptide, is shown on the right. Parts of the precursor protein flanking the mature domain are shown in lighter shading. Figure reprinted with permission from Daly et al. ${ }^{379}$

pro-region and an N-terminal region (NTR) that is repeated in some genes along with the adjacent core peptide region, as illustrated in Fig. 25.

\subsection{Biological activities of cyclotides}

Cyclotides are thought to be plant defence molecules, given their potent insecticidal activity against Helicoverpa species. ${ }^{380-383}$ However, they also have a broad range of other biological activities, including anti-HIV, ${ }^{384}$ antimicrobial, ${ }^{385,386}$ cytotoxic, ${ }^{387}$ molluscicidal, ${ }^{388}$ anti-barnacle, ${ }^{389}$ nematocidal, ${ }^{390,391}$ and haemolytic activities. ${ }^{392}$ Some of these activities are of potential pharmaceutical interest, and because of their exceptional stability, cyclotides have also attracted attention as potential protein engineering or drug design templates. ${ }^{393,394}$

The diverse range of activities of cyclotides seems to have a common mechanism that involves binding to and disruption of biological membranes. ${ }^{395}$ For example, electron micrographs of Helicoverpa larvae fed a diet containing cyclotides at a similar concentration to that which occurs naturally in plants show marked swelling and blebbing of mid-gut cells. ${ }^{380}$ Larvae that have ingested cyclotides are markedly stunted in their growth and development, presumably as a result of this disruption of their mid-gut membranes. A range of biophysical studies ${ }^{396-398}$ have confirmed membrane interactions for both Möbius and bracelet cyclotides, and detailed information is available on the residues involved in making contact with membrane surfaces. Furthermore, electrophysiological and vesicle leakage studies have confirmed the leakage of marker molecules through membranes treated with cyclotides. It appears that cyclotides are able to self-associate in the membrane environment to form large pores.

\subsection{Cyclotide biosynthesis}

As indicated in Fig. 25, cyclotides are derived from precursor proteins that encode one or more copies of the core peptide sequences, similar to the precursor peptides for cyanobactins (section 6) and some orbitides (section 16.3). For example the Oak1 (Oldenlandia affinis kalata B1 gene) encodes an $11 \mathrm{kDa}$ precursor protein that contains an endoplasmic reticulum (ER) signal, leader peptide, kalata B1 core peptide, and a C-terminal peptide region, whereas the Oak4 gene encodes a precursor containing three copies of kalata B2 (Fig. 25). ${ }^{382}$ The single or multiple copies of the cyclotide domains are flanked in each case by $\mathrm{N}$-terminal and $\mathrm{C}$-terminal recognition sequences that are thought to be important for the processing reactions.

Because of the presence of the ER signal it is believed that cyclotide precursors are probably folded in the ER prior to processing (excision and cyclization) of the cyclotide domain. Protein disulfide isomerases (PDIs) are often involved in the folding of disulfide-rich proteins ${ }^{399}$ and in vitro experiments have shown increased yields of folded cyclotide kalata B1 in the presence of PDI, ${ }^{399}$ although so far there have been no definitive studies on the involvement of PDI for in planta cyclotide folding. A recent study has shown that cyclotides are targeted to vacuoles in plant cells and this is where the excision and cyclization processes are thought to occur. ${ }^{\mathbf{4 0 0}}$

The N-terminal repeat (NTR) region of cyclotide precursors has been found to adopt an $\alpha$-helical structure as an isolated synthetic peptide, ${ }^{62}$ but the significance, if any, of this is not yet known. It has been proposed that it might represent a recognition sequence for cyclotide folding by PDI, but, interestingly, cyclotide precursors from the Fabaceae plant Clitorea ternatea lack the NTR region seen in Violaceae or Rubiaceae precursors. The nature of the processing reaction(s) occurring at the $\mathrm{N}$ terminal end of the cyclotide domain is still essentially unknown.

However, there is strong evidence that asparaginyl endoprotease (AEP) activity is responsible for processing at the Cterminal end of the cyclotide domain including involvement in the cyclization process. ${ }^{\mathbf{4 0 1 , 4 0 2}}$ With just one exception (circulin F) all cyclotides have an Asn or Asp residue as the C-terminal end of the core peptide, and an AEP is in principle able to cleave after this residue. It has been proposed that this cleavage reaction occurs contemporaneously with ligation to the earlierreleased N-terminus of the core peptide region. Mutagenesis experiments in transgenic tobacco and Arabidopsis plants have shown a vital role for the $\mathrm{N}$-terminal Asn residue, as well as other key flanking residues. ${ }^{\mathbf{4 0 1}}$ Similarly, gene silencing experiments in which AEP activity is knocked down show decreased 
production of cyclic peptide in transgenic plants. ${ }^{402}$ Overall, the processing of cyclotides can be summarized as involving excision and cyclization from a pre-folded precursor.

\subsection{Specific recommendations for the cyclotide family}

At present there is no common gene nomenclature for cyclotide precursors and given the limited knowledge of the full complement of associated biosynthetic genes encoding proteins involved in processing it seems premature to propose one. A naming scheme for the mature cyclic peptides that involves an indicative and pronounceable acronym based on the binomial name of the plant species in which the cyclotide was first discovered has been proposed, ${ }^{403}$ but has not been uniformly followed so far. We recommend that this suggestion be followed for individual peptide names; this does not exclude the additional use of 'family' names for subgroups of similar cyclotides, for example the cycloviolacins. ${ }^{404}$

\section{Orbitides: plant cyclic peptides lacking disulfide bonds}

\subsection{Perspective}

In 1959, Kaufman and Tobschirble ${ }^{405}$ reported the discovery of a nonapeptide present in a slime that had precipitated from a container of flaxseed oil. Curiously, this peptide composed of proteinogenic amino acids was cyclized by an N-to-C terminal amide bond. Cyclolinopeptide A, cyclo-[ILVPPFFLI], was the first of more than 168 peptides discovered in plants with similar structures. ${ }^{198,406}$ Small plant cyclic peptides consisting entirely of alpha-amide linkages of natural amino acids were called Caryophyllaceae-like homomonocyclopeptides in a recent review. ${ }^{198}$ It is suggested here that the name orbitides be used to refer to all N-to-C cyclized peptides from plants that do not contain disulfides. Orbitides are made by at least nine individual plant families including the Annonaceae, Caryophyllaceae, Euphorbiaceae, Lamiaceae, Linaceae, Phytolaccaceae, Rutaceae, Schizandraceae, and Verbenaceae. The peptides range in size from five to twelve amino acids. It is notable that two-amino-acid cyclic peptides from plants have been observed but it is uncertain whether these arise from a ribosomal precursor.

The 98 Caryophyllaceae-like non-redundant orbitides represented on cybase.org.au collectively contain 719 amino acids (Spring 2012). Based on simple statistics, many amino acids are highly underrepresented. Unlike most of the RiPPs discussed in this review, cysteine is the least abundant amino acid observed in orbitides as it appears just twice in a single core peptide predicted based on homology with a precursor EST sequence. Acidic residues (Asp, Glu) and their amides (Asn, Gln), basic residues (Lys, Arg), and His are also rare. Methionine, while rare overall, is well represented in the amino acids of currently known orbitides from Linum usitatissimum. Clearly, the amino acid composition of the orbitides described to date is significantly biased towards hydrophobic amino acids. Glycine and proline are found in most peptides, and serine, threonine and tyrosine occur 25,31 , and 54 times respectively.

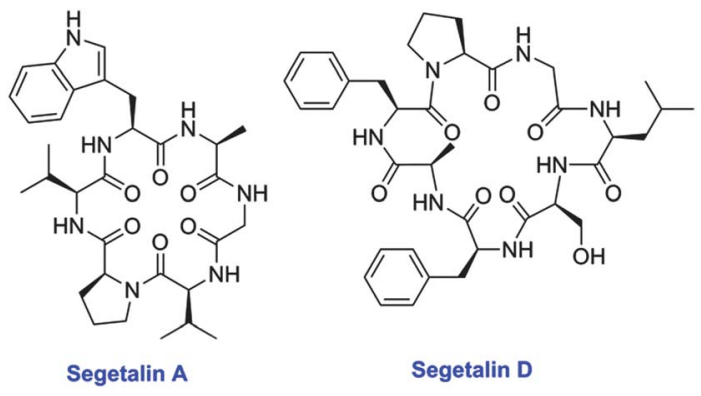

Fig. 26 Structures of segetalins containing six and seven amino acids, isolated from Vaccaria segetalis (Caryophyllaceae). ${ }^{\mathbf{0 0 6}}$

Orbitides are composed largely of unmodified L-amino acids (e.g. Fig. 26) although there are several exceptions. ${ }^{198}$ Methionine oxidation is frequently observed in these peptides, but it has not been determined whether this is a true modification or an artifact of isolation. Determination of the stereochemistry of the sulfoxide by amino acid analysis may be able to address this question. Hydroxylated amino acids, including gamma-hydroxy isoleucine and delta-hydroxy-isoleucine ${ }^{407,408}$ have also been observed as well as an unusual $N$-methyl-4-aminoproline present in cyclolinopeptide X. ${ }^{409}$ Additionally, D-tryptophan was observed in a peptide discovered in Schnabelia oligophylla. ${ }^{410}$

The rigid structure of orbitides and low amino acid complexity has enabled extensive structural studies. ${ }^{198}$ They have been crystallized from organic solvents, and solution structure information has been obtained using CD, NMR spectroscopy, and other methods. The published three-dimensional structures show a single stable conformation for most orbitides. Prolyl residues may be either in the cis or trans conformation depending on the peptide, and structures are typically stabilized by internal hydrogen bonds between amide backbone atoms. ${ }^{198}$

Only recently has it been shown that these peptides are derived from post-translational modification of ribosomally synthesized precursor peptides. ${ }^{411}$ Based on observations of the apparent segregation of segetalin peptides in closely related varieties of Vaccaria hispanica and the segregation of peptides in double haploid lines derived from crosses, it was reasoned that a RiPP origin was possible. A strategy for searching for the genes encoding these compounds was developed based on sequence tags obtained primarily by tandem mass spectrometry. The $\mathrm{N}$ or C-terminus of the linear precursor peptide cannot be determined from the amino acid sequence analysis of the cyclized peptide, therefore, EST libraries were searched for all possible linear sequences of a peptide. ${ }^{411}$ Positive hits were found for cyclolinopeptide G, F and D (Fig. 27) as well as a number of segetalin orbitides. Previously these sequences had been disregarded due to their small size relative to other sequences. Expression of a cDNA encoding the precursor sequence of segetalin A in transformed $S$. vaccaria hairy roots from the variety White Beauty, which is unable to produce segetalin A, led to the production of the peptide. ${ }^{411}$ Furthermore, extracts of developing S. vaccaria seeds were shown to catalyze the production of segetalin A from a synthetic peptide precursor 


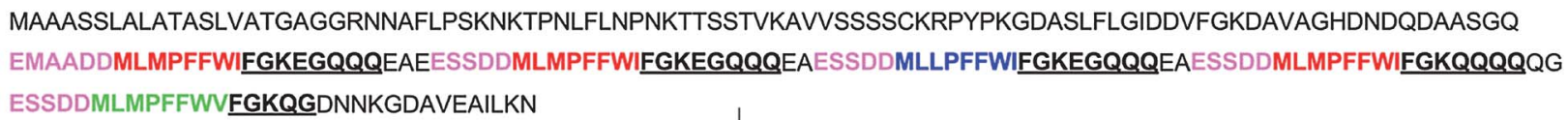
ESSDDMLMPFFWVFGKQGDNNKGDAVEAILKN

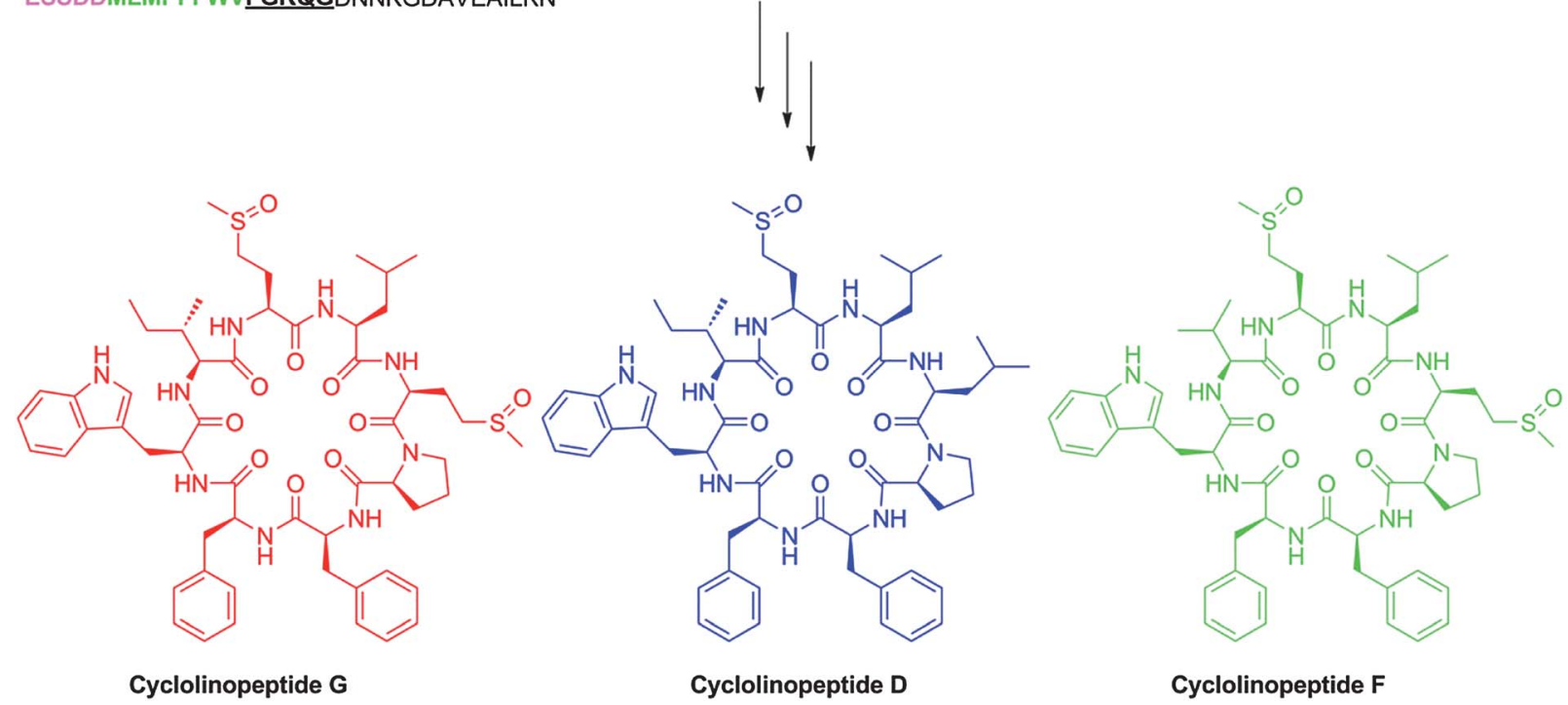

Fig. 27 Amino acid sequences of cyclic peptides arising from Linaceae species Linum usitatissimum. The RiPP core peptides putatively encoding cyclolinopeptides D, F, and $\mathrm{G}$ is shown in blue, green, and red, respectively, with putative recognition sequences shown in pink and underlined font.

representing the gene product. Moreover, the presence of two segetalins, J [cyclo-(FGTHGLPAP)] and $\mathrm{K}$ [cyclo-(GRVKA)] were predicted by sequence analysis of $S$. vaccaria cDNA. A focused search by liquid chromatography/mass spectrometry confirmed the existence of these compounds. Sequence analysis also predicts the presence of similar precursor genes in Dianthus caryophyllus, Citrus spp., Linum usitatissimum, Gypsophila arrostil, and Jatropha spp.

\subsection{Biosynthesis of orbitides}

Searches of EST libraries derived from members of the family Caryophyllaceae has yielded gene sequences that appear to encode the precursor peptides. ${ }^{411}$ To date such sequences have only been observed in Caryophyllaceae mRNA as no genome databases of Caryophyllaceae species are available. The mRNA sequences are ubiquitously expressed in many plant tissues and the copy number of these sequences is high relative to other genes. The mechanism of processing of the precursor peptides is not known at this time. They typically contain a leader peptide, one core peptide, and a recognition sequence (Table 2). Caryophyllacaceae orbitide precursor sequences observed thus far exhibit a highly conserved recognition sequence with nine invariant residues. mRNA sequences of Dianthus caryophyllus and Gypsophila arrostil encode conservative recognition sequences that are highly similar, but identification of the cyclic peptides is necessary to confirm that the mRNAs lead to peptide products. Curiously, an EST sequence from Gypsophila arrostil contains stop codons and may be a pseudogene (Table 2). The leader sequences of all observed mRNA sequences that potentially encode RiPPs of caryophyllaceae orbitides contain 13 amino acids (Table 2).

The sequences of Rutaceae precursor peptides are known from both mRNA and genomic DNA as genome sequences are available for Citrus sinensis ${ }^{\mathbf{4 1 2}}$ and Citrus clementina. ${ }^{\mathbf{4 1 3}}$ The observed DNA sequences are short and there are many more potential RiPP coding sequences than known peptides. The recognition sequence is highly conserved in both length and amino acid composition (Table 2), whereas the leader sequences are more variable.

\subsection{Biosynthesis of orbitides in Linaceae}

The genomic and mRNA sequences from the flaxseed variety Linum usitatissimum cv Bethune have been determined. ${ }^{\mathbf{4 1 4 4 1 5}}$ Blast searches of EST sequences derived from this cultivar using a search string based on the known amino acids of cyclolinopeptide $\mathrm{G}$ revealed a sequence that potentially includes five core peptides in a single precursor peptide (Fig. 27). ${ }^{416}$ Three of these sequences corresponded to cyclolinopeptide G. The ability of a single precursor to be processed to multiple cyclic peptides is unique among known precursor peptides of orbitides but is also found in the precursors to cyclotides (section 15), cyanobactins (section 6), and possibly microviridins (section 11). A search of a genome database that comprised shotgun sequences of Bethune flax using the polyRiPP precursor sequence revealed both the DNA sequence that encodes cyclolinopeptides D, F and G (Fig. 27) as well as an additional sequence that encodes a polyRiPP precursor that may be processed to cyclolinopeptides A, B and E. ${ }^{\mathbf{4 1 6}}$

\subsection{Biosynthesis of orbitides in Euphorbiaceae}

The interest in Jatropha curcas as a potential biofuel crop has resulted in generation of a substantial database of EST sequences and a genomic sequence is also available. ${ }^{417} \mathrm{~A}$ search of EST libraries produced from Jatropha curcas conducted for 
Table 2 Predicted amino acid sequences of cyclic peptides from Caryophyllaceae and Rutaceae

\begin{tabular}{|c|c|c|c|}
\hline Leader & Core peptide $^{a}$ & Recognition sequence $^{b}$ & Name \\
\hline \multicolumn{4}{|l|}{ Caryophyllaceae } \\
\hline MSPILAHDVVKPQ & GVPVWA & FQAKDVENASAPV & $\mathrm{A} 1$ \\
\hline MSPILAHDVVKPQ & GVAWA & FQAKDVENASAPV & B1 \\
\hline MSPIFAHDVVNPQ & GLSFAFP & AKDAENASSPV & D1 \\
\hline MSPIFVHEVVKPQ & GVKYA & FQPKDSENASAPV & G1 \\
\hline MSPIFAHDIVKPK & GYRFS & FQAKDAENASAPV & $\mathrm{H} 1$ \\
\hline MSPILALDRYKPE & GRVKA & FQAKDAENASAPV & K1 \\
\hline MSPILSHDVVKPQ & GLPGWP & FQAKDVENASAPV & L1 \\
\hline MATSFQFDGLKPS & FSASYSSKP & IQTQVSNGMDNASAPV & F1 \\
\hline MATSFQLDGLKPS & FGTHGLPAP & IQVPNGMDDACAPM & J1 \\
\hline MXPTVAHNMLKPH & GSIFES & FQAKDAENXSAPV & JK743974 \\
\hline
\end{tabular}

Rutaceae

\begin{tabular}{rll}
\hline-1 & 1 & +1 \\
METTCAGNNW SE & GLLLPPFG & SIADDDVMND NLDFLNVPQYGRNPDYMG \\
MKTLAGAGMSDPSE & GLVLPS & SIADDDVGND NLDLIVIPQYGRNPDYYG \\
MKTLPGAGMSDPSE & GYLLPPS & SIADDDVGND NLDLIVIPQYGRNPDYYG \\
MKIMETTCAGNDDCLE & GRPWNLA & SIVDDNVANDVNLDLLAVPQYGRNDQTG \\
MKNMETTSAGNDDWLE & GYVAA & SIVDDNIANDVNLDLLTVPQYGRNIDQTG \\
MKNMETTCAGNDDWLE & GAPWLIAA & SIVDDNIANDVNLDLLTVPQYGRNIDQTG \\
MKNMETTSAGNADWLE & GVPWAIAA & SIVDDNIANDVNLDLLTVPQYGRNIDQTG
\end{tabular}

${ }^{a}$ Products of bolded core peptides have been observed by MS/MS. ${ }^{b}$ Amino acid X reflects a stop codon within the sequence.

this review identified two mRNA sequences that encode the two known cyclic peptides previously isolated from $J$. curcas, curcacyclines A and B. ${ }^{418}$ These sequences have no C-terminal recognition sequence as the gene terminates with the core peptide, thus requiring activation of the C-terminal carboxylate, similarly to the bacterial N-to-C cyclized peptides discussed in section 13. The leader sequences of both peptides contain 36 amino acids and are highly conserved (Table 3). Many other cyclic peptides have been isolated from Jatropha, ${ }^{419}$ and may have a RiPP origin.

\subsection{Relationship to other RiPP subfamilies}

Orbitides have been observed in a range of plant tissues including roots, stems, leaves, developing seeds, mature seeds, fruits, and peels, ${ }^{198}$ and the corresponding mRNA has been detected in the various plant tissues. To date over one hundred unique peptides have been discovered, but only a small number of the species that are likely to produce them have been surveyed. Combined the families of Rutaceae, Linnaceae, Caryophyllaceae, Verbenacea, Annonaceae, Lamiaceae, Phytolaccaceae, Euphorbiaceae, and Schizandraceae have thousands of members. ${ }^{420}$ It is probable that a thorough study will reveal hundreds if not thousands of additional peptides.

Although the cyclotides and orbitides appear to be quite different in size, structure, and primary composition there is a striking similarity in gene organization between the cyclotides and the orbitides from flax (cyclolinopeptides). Genes in both groups comprise, at the carboxy terminal end of the gene, multiple copies of the cyclic peptide sequences flanked by

Table 3 Predicted amino acid sequence of Jatropha cyclic peptides based on genome sequences identified in Genbank. The sequences corresponding to curcacyclines $A$ and $B$ are shown in red font

BABX01100257.1 (3315-3349) MAVSPIQSVLGMLGVMSMQQRQAGVNISSFYPPVAQ ILGSPILIG BABX01004419.1 (3001-2870) MAVSPIQSVLGMLGVMSTAQRQTGVNISSFYPPVAQ LIGTVL-IG BABX01106530.1 (407-535) Consensus MAVSPIQSVLGMLGIMSMQHRHAGVHISSFYPPVAQ VWNSWFNMAVSPIQSVLGMLG-MS---R--GV--SSFYPPVAQ ------ 
highly conserved regions. In contrast DNA sequences that comprise orbitides from Caryophyllacea genera such as Vaccaria and Rutaceae genera such as Citrus comprise the highly conserved flanking sequences but contain only a single cyclopeptide sequence.

This apparent difference in gene structure could result from the fact that the published Vaccaria and Citrus sequences were only derived from mRNA and as such may only represent pieces of pre-mRNA from a more complex gene. This possibility is supported by the fact that the majority of these DNA sequences are lacking a methionine amino terminus of the precursor peptide and the cDNAs of those that do have an intact amino terminal portion do not have features such as a tata-box expected in a promoter. The sequences flanking the precursor peptides have a high AT content and features typical of introns.

It is noteworthy that despite the relatively extensive size of the flax EST library and the abundance of cyclolinopeptides A, C and E, the mRNA from this gene has not been found and the gene was only uncovered when the genomic sequence of flax was determined. The unusual structure of the mRNAs from these genes apparently precludes discovery by standard cloning methodology. In consideration of this issue, and very few genomic sequences that are yet available, it is possible that the organization of the genes in other plant families that are known to make orbitide type cyclopeptides will be similar to that of the cyclotides and the orbitides from flax.

\subsection{Biological activity}

The innate biological role of orbitides is currently unknown. Generally the lipophilic composition of the orbitides is consistent with a membrane active role. ${ }^{\mathbf{2 2 1}}$ Attention has largely been focused on the use of these compounds as drug candidates and in agriculture. ${ }^{198}$ Preparations containing orbitides have been tested using a number of bioassays that do not always relate to mechanism of action. For example, orbitides are classified as cytotoxic, antiplatelet, antimalarial, immunomodulating, and inhibiting $\mathrm{T}$ cell proliferation. ${ }^{198}$ These assays provide promising leads to understanding biologial activity and potential applications of the peptides, but a universal mechanism of action is unlikely. Analysis of the effects of peptides using in vitro assays has lead to a more precise understanding of the possible mechanism of action of some peptides. Curcacycline B enhances prolyl cis-trans isomerase activity of human cyclophilin $\mathrm{B},{ }^{\mathbf{4 2 2}}$ and cycloleonuripeptide $\mathrm{D}$ inhibits cyclooxygenase. ${ }^{423}$ Structure activity studies of pseudostellarin A showed that $\gamma$ - and $\beta$ - turn structures were fixed by transannular hydrogen bonds between Gly and Leu. ${ }^{\mathbf{4 2 4}}$ This rigid conformation was hypothesized to be important in tyrosinase activity exhibited by this compound. Similarly, the Trp-Ala-GlyVal sequence in segetallin A and B has been suggested to play an important role in their estrogen-like activities. ${ }^{\mathbf{4 2 5}}$

\subsection{Specific recommendations for the orbitide family}

We propose that the name "orbitide" replace the longer designation "Caryophyllaceae like homomonocyclopeptides" and is used for all plant N-to-C cyclized peptides that do not contain disulfide crosslinks. It is too early to classify the various orbitides when so few confirmed examples are available.

\section{Conopeptides and other toxoglossan venom peptides}

The venoms of predatory marine snails (such as species in the genus Conus, the cone snails) are a complex mixture of ribosomally-synthesized peptides that are post-translationally modified to various extents. ${ }^{\mathbf{4 2 6}}$ Although the possibility that these venoms may also contain gene products from bacterial symbionts cannot be completely excluded, the vast majority of peptides are encoded in the genome of the marine snail itself, expressed in the epithelial cells lining the venom duct, and secreted into the lumen of the duct. Transcriptome analyses that provide quantitative estimates of the mRNAs expressed in the snail's venom duct typically reveal that a major fraction of all transcripts encode the secreted venom peptides (see for example ref. 10, 427, and 428).

Biologically-active peptides from animal venoms are an increasingly important class of natural products. ${ }^{429}$ In total, these comprise many millions of different peptides, each presumably evolved to alter the function of a specific physiological target. Like the other RiPPs discussed in this review, venom peptides are generally post-translationally processed from larger precursors, to yield peptides that are mostly in a size range of 30-90 amino acids after processing. ${ }^{24}$ The venom peptides evolved by marine snails differ from most animal venom peptides in several distinct ways. The best characterized of the marine snail peptides, those of the cone snails (generally known as conopeptides or conotoxins) are mostly smaller (1030 amino acids), with a significantly higher density of disulfide crosslinks than most animal venom peptides. Another feature is the larger diversity of post-translational modifications in these peptides compared to other venom peptides (e.g., scorpion or snake peptides). ${ }^{426}$ This diversity of post-translational modifications make them more like the RiPPs from bacterial and plant sources.

As detailed below, marine snail venom peptides have an entirely different set of post-translational modifications compared to other RiPPs. One likely fundamental cause for the lack of overlap in PTMs is that the evolution of post-translational modification in marine snail venom peptides has followed a very different path from post-translational modifications of peptidic natural products produced by bacteria. In the evolution and adaptive radiation of venomous animals, a delivery system must be evolved; thus, venoms are intrinsically injectable mixtures of natural products. Since a delivery route has already been established when the pharmacological agents in venoms are being evolved, peptides and polypeptides that suit the biological purposes of the venomous animal can immediately be selected for. In general, there are no major barriers to delivery of peptides and polypeptides once venom is injected into the targeted animal and most molecular targets are plasma membrane proteins. Thus, post-translational modifications are presumably evolved primarily to increase potency and selectivity, rather than to improve the efficiency of 
delivery, which may have been an evolutionary driving force for bacterial RiPPs.

As a class, marine snail peptides are structurally extremely diverse, and a comprehensive discussion of all of the structural and functional subclasses that have been described would require a much longer exposition than warranted in the present overview. Venom peptides of marine snails run the gamut from ribosomally-synthesized peptides that are quite minimally posttranslationally modified, to peptidic natural products that have been subjected to more diverse post-translational modifications than most other RiPPs. The different post-translational modifications that have been reported in conopeptides to date are shown in Table 4. One feature that makes the literature on molluscan venom peptides particularly confusing compared to the other RiPPs discussed in this review is that the nomenclature in use names these peptides on the basis of their functional specificity and taxonomic origins, rather than their structural and/or chemical features.

\subsection{Biological and toxicological perspectives}

Venomous marine snails comprise a forbiddingly complex assemblage of $>10000$ species. ${ }^{430}$ Taxonomists typically refer all venomous species to a single superfamily ("Conoidea") or suborder ("Toxoglossa"), referred to as "conoideans" or "toxoglossans." This is likely the most species-rich of all molluscan lineages with a large number of extremely small, deep-water forms that mostly remain undescribed. Venoms of all toxoglossans are produced in a specialized venom duct; the analysis of any individual venom has invariably demonstrated that it is a complex mixture with essentially all of the biologicallyactive components being peptides. Although, cone snails (genus Conus or family Conidae) comprise less than $5 \%$ of the total biodiversity of venomous molluscs, greater than $99 \%$ of all published papers in the scientific literature concern peptides derived from cone snails.

A nomenclature for peptides from other lineages of venomous marine snails has recently been suggested, based on

Table 4 Modifications of amino acid residues found in conopeptides

\begin{tabular}{lll}
\hline Modification & Amino acid residue & Final product \\
\hline $\begin{array}{l}\text { C-terminal } \\
\text { amidation }\end{array}$ & Glycine & C-terminal -CO-NH ${ }_{2}$ \\
$\begin{array}{l}\text { N-terminal } \\
\text { cyclization }\end{array}$ & Glutamine & N-terminal pyroglutamate \\
Hydroxylation & Proline; Valine; & 4-Hydroxyproline; \\
& Lysine & D- $\gamma$ hydroxyvaline; \\
& & 5 -hydroxylysine \\
Carboxylation & Glutamate & $\gamma$-Carboxyglutamate \\
Sulfation & Tyrosine & Sulfotyrosine \\
Bromination & Tryptophan & 6-Bromotryptophan \\
Oxidation & Cysteine & Disulfide bridge \\
Glycosylation & Threonine & Various $O$-glycosyl amino acids \\
Epimerization & Valine; Leucine; & D-Valine; D-leucine; \\
& Phenylalanine; & D-phenylalanine; D-tryptophan \\
& Tryptophan &
\end{tabular}

family groups within the superfamily Conoidea. Thus, in addition to conopeptides from cone snails, venom peptides from species in the family Turridae are referred to as turripeptides, and from the family Clathurellidae as clathurellipeptides, etc. (12 other family groups have been proposed). ${ }^{\mathbf{4 3 1 , 4 3 2}}$ Very few venom peptides from toxoglossans other than the cone snails have been characterized, and these mostly conform to the generalizations about conopeptides that we outline below. Because conopeptides have been much more extensively investigated, our focus will be on these peptides, but the reader should be aware of the vast trove of uncharacterized, post-translationally modified peptides from the other toxoglossan lineages.

One general feature of all marine snail venoms is their complexity both in the number of different peptides found, as well as in their structures. Furthermore, the genes encoding these venom peptides undergo accelerated evolution, so that there is essentially no molecular overlap in the modified core peptides found in venoms of even closely-related cone snail species. Most genes that encode these peptides have introns, and the initial transcripts have to undergo splicing before the mRNA can be exported from the nucleus. A small minority of modified core peptides are apparently encoded by genes without introns.

The translated precursor peptides have the canonical organization characteristic of RiPPs, with the leader peptide varying considerably in size; most conopeptide precursor peptides are 70-120 amino acids, and the majority of wellcharacterized modified core peptides found in cone snail venoms fall in the range of 12-30 amino acids. One unique feature of conopeptide superfamilies is the remarkable conservation of signal sequences within all members of the superfamily, providing an unequivocal marker for the gene superfamily to which a specific venom peptide belongs. Although there may be 50-200 different conopeptides found in the venom of an individual species, the vast majority of these typically belong to only 5-8 superfamilies.

\subsection{Conopeptide structures and biological activities}

Conopeptides comprise a more or less continuous spectrum of post-translational modification, with several widely distributed in all animal venom peptides: proteolytic cleavage, disulfide bond formation, C-terminal amidation, and cyclization of $\mathrm{N}$ terminal Gln residues to pyroglutamate. Some small, disulfiderich, endogenous peptides such as endothelin are similar in their general biochemical characteristics to many conopeptides. A superfamily of larger conopeptides (called conkunitzins) have clearly co-opted a well-characterized endogenous structural framework known as the Kunitz domain, which is widely found in protease inhibitors such as bovine plasma trypsin inhibitor (BPTI). Some venom peptides are expressed in other tissues of the cone snail, presumably with endogenous physiological functions (e.g., the conopressins). However, the origin of the majority of conopeptide superfamilies has not been established and in contrast to snake toxins and scorpion peptides that have 1-2 conserved structural frameworks, each gene superfamily represents at least one distinctive structural fold. 
Many conopeptides have a surprising array of diverse covalent modifications, each carried out by a different post-translational modification enzyme. One notable difference from RiPPs from bacteria and archaea is that they are encoded by eukaryotic genes that are not organized in a biosynthetic gene cluster. Adding to the complexity is that in a number of cases, peptides that are post-translationally modified at a particular locus are found in the venom along with the corresponding peptide with the identical amino acid sequence that has not been post-translationally modified. This trend is particularly observed for hydroxylation of proline to 4-hydroxyproline. The functional significance of the incomplete penetrance of some post-translational modifications has not been critically assessed.

For most conopeptides, the most important structural feature that confers the correct three-dimensional conformation to the biologically-active peptide are disulfide bonds. Conopeptides can have an unprecedented density of disulfide crosslinks; some conopeptides are only twelve amino acids in length, with three disulfide crosslinks. Since six Cys residues forming three disulfides can generate fifteen distinct isomers, the oxidative folding pathway of an unmodified core peptide to the specific biologically-active disulfide crosslinked isomer may well be enzyme catalyzed. ${ }^{433}$ This "conotoxin oxidative folding problem" is a challenging issue that has not been mechanistically elucidated, and it is likely that formation of the correct disulfide crosslinks in conotoxins requires special post-translational enzymes and accessory factors.

The pharmacological characterization of conopeptides to date has demonstrated great diversity of targets. A large majority of the molecular targets of conopeptides that have been elucidated are ion channels and receptors present on the plasma membrane; many are expressed in nervous systems. Thus, conopeptides have become a mainstay of ion channel pharmacology, ${ }^{\mathbf{4 3 4}}$ and are widely used as basic research tools in neuroscience.

It is perhaps easiest to illustrate the range of conopeptide functional and structural diversity by focusing on their direct biomedical applications. At present, several conopeptides have been developed as lead compounds for therapeutic drugs, and one conopeptide is an approved commercial drug in the U.S. and European community (Prialt, for intractable pain). Some of the conopeptides that have reached human clinical trials are shown in Fig. 28. ${ }^{435}$ They demonstrate the structural and functional diversity in this compound family.

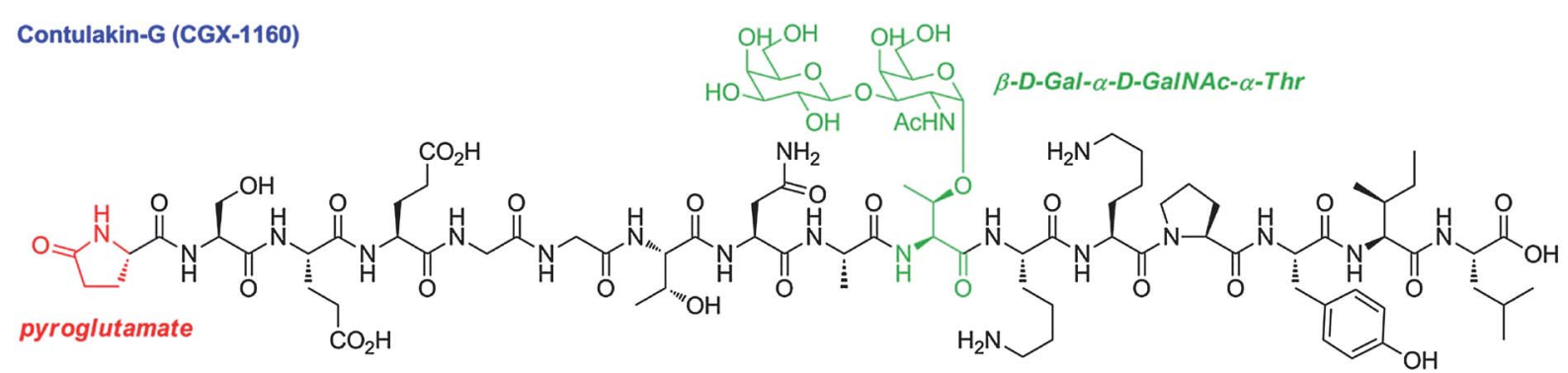

Conantokin-G (CGX-1007)

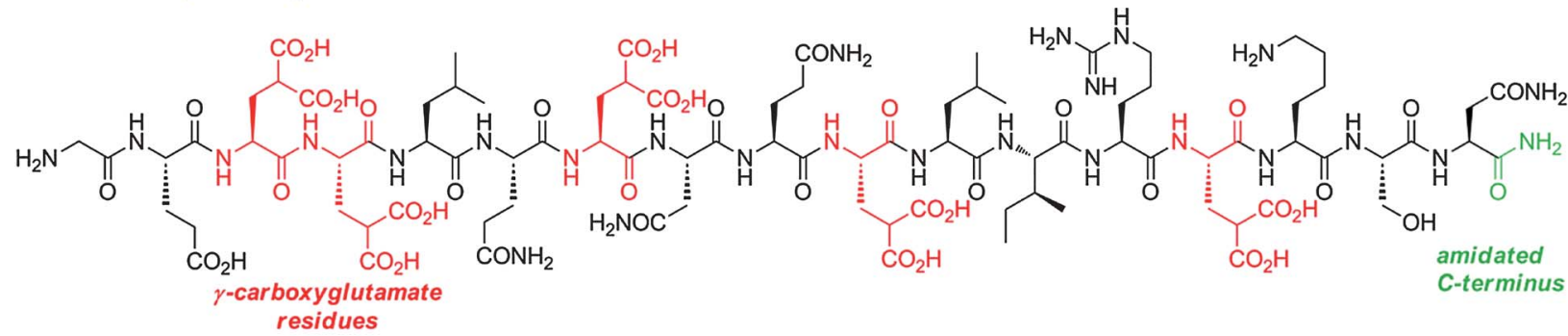

Modified $\chi$-conopeptide MrIA (XEN-2174)

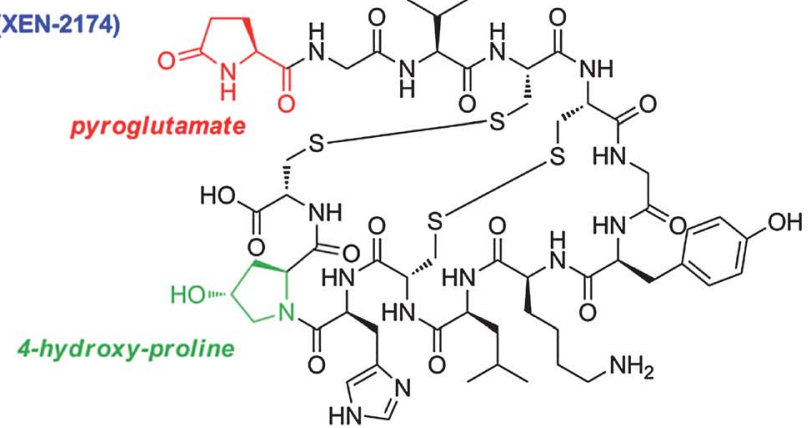

Fig. 28 Structures of three post-translationally modified conopeptides that reached human clinical trials. 
Two of the peptides in Fig. 28, contulakin G and conotokin $\mathrm{G}$, both characterized from the venom of Conus geographus, reached Phase I clinical trials for pain and epilepsy, respectively. Contulakin $\mathrm{G}$ has a glycosylated threonine residue that is essential for its potent analgesic activity. It is targeted to a Gprotein coupled receptor (the neurotensin receptor) and has striking sequence homology at its C-terminal end to neurotensin. Interestingly, although the glycosylation makes the peptide less potent as an agonist at the neurotensin receptor, it renders the peptide a more effective analgesic. Fig. 28 also shows the structure of a modified conopeptide $\chi$-MrIA that is in clinical development and inhibits the norepinephrine transporter; this analgesic peptide has been re-engineered by chemists to introduce a modification (pyroglutamate) in addition to the preexisting two disulfide bridges and 4-hydroxy-Pro found in the native peptide isolated from Conus marmoreus.

\subsection{The conantokin superfamily and $\gamma$-carboxylation of glutamate}

Although the three-dimensional conformation of most modified conopeptides is determined by the pattern of disulfide crosslinking, a notable exception are members of the conantokin superfamily, including conantokin G (Fig. 28). ${ }^{436}$ Conantokin $\mathrm{G}$ is an NMDA receptor antagonist, highly selective for one particular subtype of this receptor (i.e., receptors with NR2B subunits). For all conantokin peptides, an unusual post-translational modification, the carboxylation of glutamate residues to form $\gamma$-carboxyglutamate ( $\gamma$ or Gla) is required. When these modified amino acids are spaced every three or four residues apart in the primary amino acid sequence, the peptide folds into a stable helical conformation in the presence of $\mathrm{Ca}^{2+} \cdot{ }^{437}$ In essence, the modification of glutamate to $\gamma$-carboxyglutamate creates a functional group for coordinating $\mathrm{Ca}^{2+}$; Two adjacent Gla residues on the same side of a helix chelate $\mathrm{Ca}^{2+}$ in a manner analogous to EDTA or EGTA. Thus, the post-translational modification of glutamate residues by the enzyme $\gamma$ glutamyl carboxylase is essential for the biologically-active conformation of conantokin G.

A similar motif is found in many of the proteases comprising the mammalian blood-clotting cascade (such as prothrombin). The presence of a domain containing Gla residues is essential for the accumulation of blood-clotting factors on the membranes of activated platelets, thereby triggering the formation of blood clots. However, in mammals this modification is only found in large proteins. Among RiPPs, the $\gamma$ carboxylation of glutamate appears to be unique to toxoglossan venom peptides (the presence of this modification has been established both in conopeptides, and in certain turripeptides from venomous snails in the family Turridae).

\subsection{Biosynthesis of conopeptides}

The enzyme converting glutamate to Gla is a highly-conserved, widely-distributed enzyme. ${ }^{\mathbf{4 3 8 , 4 3 9}}$ Not only do the enzymes that carry out this modification in cone snails and in the mammalian blood-clotting system have high sequence similarity, they are also functionally homologous-both require reduced vitamin $\mathrm{K}$ as a cofactor, and are stimulated by binding to a recognition sequence that determines which glutamate residue will be modified. An even more unexpected and striking piece of evidence for the common evolutionary origin of these enzymes was the discovery that the ten different introns in the human gene are completely conserved in their location in the Conus gene. Thus, if an intron is found between codons in the human gene, it is also found between the homologous codons in Conus. If an intron is between the second and third nucleotide of a codon in the human gene, it is in precisely the same location as the homologous codon in the Conus gene sequence. The introns themselves have no detectable sequence similarity, and the cone snail introns are longer than those in humans.

Interestingly, the same enzyme was identified in Drosophila (where its function is unknown); in the Drosophila gene only three introns remain (all at precisely conserved locations), and they are all shorter than either the corresponding mammalian or Conus introns. Thus, in this instance, the post-translational modification enzyme that the cone snails have recruited for one gene superfamily of RiPPs is clearly an ancient enzyme with its origins early in metazoan evolution. It has been speculated that because the post-translational modification promotes the formation of short $\alpha$-helices, that the carboxylation of glutamate is a relict of a pre-cellular, pre-ribosomal biotic world. This one case of a wellcharacterized post-translational enzyme that acts on conopeptides suggests that the PTMs used in marine snail venoms were already present early in evolution, and subsequently recruited in the snails venom duct for novel biological purposes.

\section{Glycocins}

The glycocins are a small group of glycosylated antimicrobial peptides produced by bacteria. The two currently characterized members, sublancin 168 produced by Bacillus subtilis 168 and

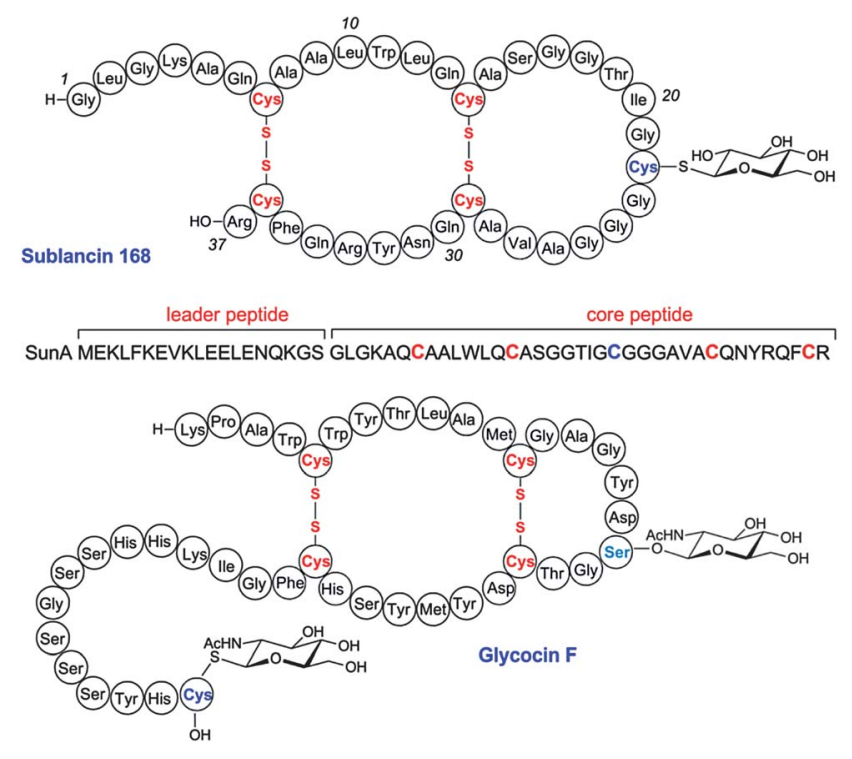

Fig. 29 Structures of two glycocins. The precursor peptide that is transformed into sublancin by disulfide formation and S-glycosylation is shown below the sublancin 168 structure. 
glycocin F produced by Lactobacillus plantarum KW30, are both glycosylated on Cys residues, ${ }^{\mathbf{4 0 0 4 4 1}}$ a very unusual form of glycosylation. In sublancin, a glucose is attached to one of five Cys residues in the precursor peptide (Cys22) with the other four Cys residues engaged in two disulfides (Fig. 29). Glycocin F has a very similar arrangement of disulfide bonds as well as a connecting loop that is glycosylated, but an $\mathrm{N}$-acetylglucosamine is conjugated to a Ser in this loop rather than to a Cys residue. The $S$-linked glycosylation in glycocin $\mathrm{F}$ is located on a Cys that is the last residue of the peptide. The NMR structure of glycocin $\mathrm{F}$ has been reported showing two alpha helices held together by the two disulfide linkages. ${ }^{442}$ These two helices also appear to be the recognition elements for the $S$-glycosyltransferase SunS involved in sublancin formation. ${ }^{443}$ This glycosyltransferase has specificity for Cys because a Cys22Ser mutant of the SunA precursor peptide was not accepted as substrate. $^{\mathbf{4 4 0}}$ However, the enzyme was promiscuous with respect to its nucleotide-sugar donor and could add a variety of different hexoses to Cys22. Furthermore, Suns glucosylated mutant peptides in which the position of the Cys was varied along the loop connecting the two helices. ${ }^{\mathbf{4 3}}$ Hence, it appears that as long as one or more Cys residues are present in this loop, SunS will carry out the $S$-linked glycosylation. Unlike most biosynthetic enzymes discussed in this review, Suns does not require the leader peptide for its activity. ${ }^{\mathbf{4 4 0}}$ Whether one or more glycosyltransferases are responsible to install the two glycosylations in glycocin $\mathrm{F}$ is currently not known. Furthermore, the mode of action of sublancin 168 and glycocin $\mathrm{F}$ has yet to be established.

\subsection{Recommendations for the glycocin family}

The glycocin family currently encompasses both $O$ - and $S$-linked bacterial glycopeptides with antimicrobial activities. Although at present both members contain $S$-linked sugars, the name glycocin is not limited to $S$-linked glycopeptides as future studies may reveal new members that only contain $O$-linked and/or $N$-linked carbohydrates. The suffix -cin in glycocin presently limits the family to antimicrobial peptides. It is possible that additional bacterial glycosylated peptides with other functions are awaiting discovery in which case a broader nomenclature may be required, similar to the extension of lantibiotics to lanthipeptides and sactibiotics to sactipeptides. The designation " $\mathrm{A}$ " is recommended for the precursor peptides and the designation "S" for the glycosyltransferase, "I" for selfresistance proteins, " $\mathrm{T}$ " for transporters, and " $\mathrm{B}$ " for thioldisulfide isomerases.

\section{Autoinducing peptides, ComX, methanobactin, and $N$-formylated peptides}

The autoinducing peptides (AIPs) are quorum sensing peptides produced by most members of the low $\mathrm{G}+\mathrm{C}$ Gram-positive bacteria (Firmicutes). ${ }^{\mathbf{4 4 4 - 4 4 6}}$ In all of these AIP signals, the presence of a cyclic ester or thioester is a conserved feature, which differentiates the AIP structures from linear regulatory peptides. The Staphylococcus aureus AIPs have received the most

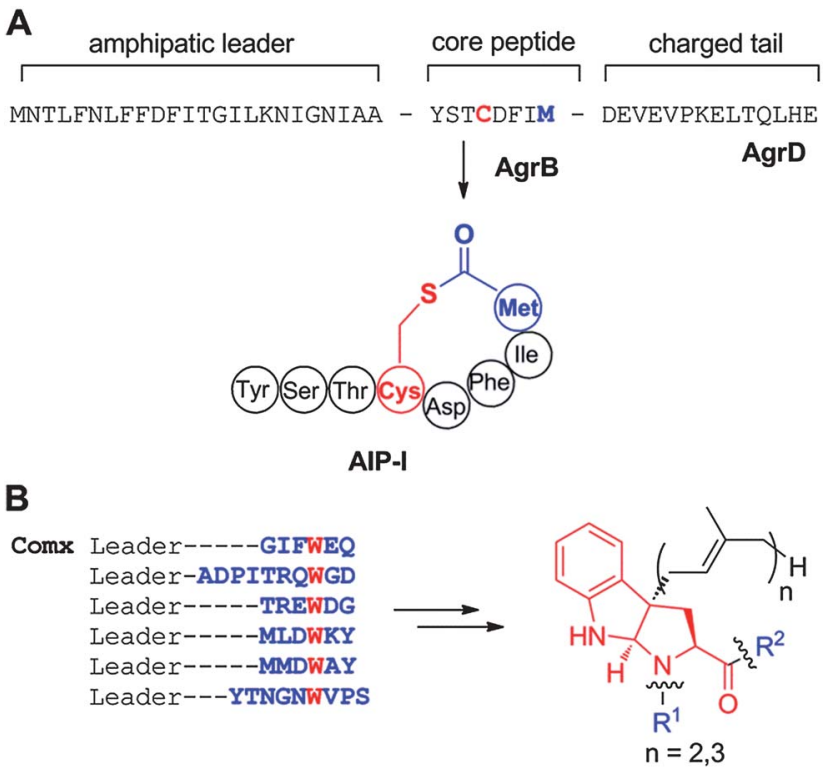

Fig. 30 A. Structure of AIP-I and the ribosomally synthesized precursor peptide from which it is produced. B. Structures of ComX and its derivatives and the ribosomally synthesized precursor peptides from which they are produced. The red font Trp is converted to the structure shown by cyclization of the amide nitrogen of Trp onto C2 of the indole and prenylation at C3. After leader peptide removal, the modified Trp is present in short peptides with the sequences of $R^{1}$ and $\mathrm{R}^{2}$ shown in blue font.

attention, and in producing strains the extracellular AIP binds to a receptor on a cognate AgrC histidine kinase, inducing a regulatory cascade that increases expression of virulence factors. ${ }^{47}$ The $S$. aureus thiolactone-containing peptide is generated from a larger precursor peptide AgrD (Fig. 30A) by AgrB, ${ }^{448,449}$ a unique transmembrane protein that has endopeptidase activity. ${ }^{\mathbf{4 4 9 - 4 5 1}}$ The molecular details of the thiolactonization process are currently not known but the general process of excision of a cyclic peptide from a linear precursor has similarities with the cyclization processes described for the cyanobactins, amatoxins, cyclotides, and some orbitides. The ArgD peptides are about 45 amino acids in length and contain three different regions: an N-terminal amphiphatic helical region, ${ }^{452}$ a core peptide that encodes the residues that will form the mature AIP, ${ }^{\mathbf{4 4 8}}$ and a C-terminal, highly negatively charged region. ${ }^{449}$ The N-terminal helix is thought to be important for interaction of the peptide with the membrane and with AgrB. Overall, there is considerable variation in the sequences of the three regions in different species with only the Cys required for thiolactone formation (or Ser for lactone formation) conserved in the core peptide. The negatively charged C-terminal putative helix is more conserved than the N-terminal helix. ${ }^{449}$

Another example of ribosomally synthesized quorum sensing compounds are ComX and its derivatives produced by bacilli. These compounds are biosynthesized from a much longer precursor peptide that contains a leader peptide and a variable core peptide (Fig. 30B). After unusual prenylation and cyclization steps involving a fully conserved Trp residue (Fig. 30B), ${ }^{453}$ the leader peptide is released and the mature compound is produced. ${ }^{454}$ 

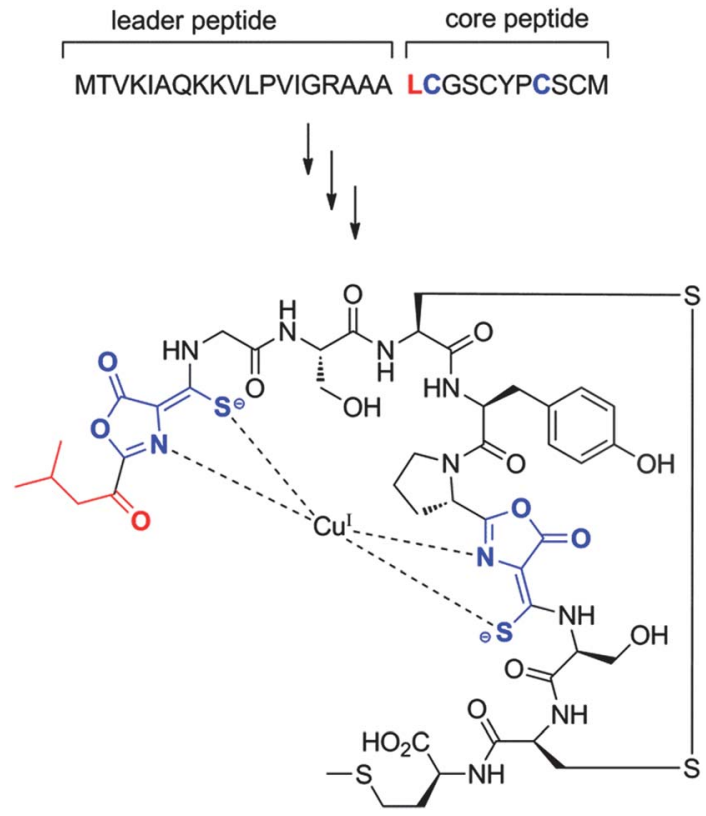

Fig. 31 Currently accepted structure of methanobactin from $M$. trichosporium $\mathrm{OB} 3 \mathrm{~b}$ and the precursor peptide from which it may be derived.

Methanobactins are copper chelators, or chalkophores $(c f$. siderophores for iron chelators) that are used by methanotrophic bacteria that use methane as their sole carbon source. Copper acquisition is critical for these Gram-negative bacteria as methane oxidation is carried out by a copper-dependent monooxygenase. ${ }^{455}$ The initially reported structure of the methanobactin isolated from Methylosinus trichosporium OB3b determined by X-ray crystallography ${ }^{456}$ has been revised on the basis of NMR studies and more recent high resolution X-ray structures (Fig. 31). ${ }^{457,458}$ It contains two oxazolones and a disulfide. When the genome of $M$. trichosporium was sequenced, a small gene was identified that encodes for a peptide that could serve as the precursor for methanobactin (Fig. 31). The peptide contains a putative 19-amino acid leader peptide and a 11amino acid core peptide. ${ }^{459}$ If this is indeed the precursor to methanobactin, post-translational modifications must involve transformation of two of the Cys residues to the oxazolones with an adjacent thioamide, deamination of an N-terminal Leu, and formation of the disulfide. Interestingly, homologous precursor peptide genes and flanking genes occur outside the methanotrophs, such as in the rice endophyte Azospirillum sp. B510.459 Further studies may provide insights into this fascinating system.

Peptidogenomic approaches have uncovered several short $\mathrm{N}$ formylated linear peptides. ${ }^{6}$ Bacterial proteins are typically formylated on the N-terminal Met residue, because translation initiates with formyl-methionine. The formyl group is usually removed by a peptide deformylase, but in some short peptides, the deformylation step appears to be incomplete or omitted altogether. In most cases, the functional relevance is unclear and the lack of deformylation may simply be the result of translation termination before the short peptide emerges from the ribosome to be deformylated. But in the case of the formylated $\delta$-toxin of Staphylococcus aureus, deformylation appears to be inhibited by depletion of iron during stationary phase. ${ }^{460}$ Furthermore, the formylated toxin, as well as other $N$ formylated peptides, ${ }^{\mathbf{4 6 1}}$ serves as a chemoattractant for neutrophils. ${ }^{\mathbf{4 6 0}} \mathrm{N}$-Terminal formylation is also found in microcin $\mathrm{C}$ discussed above. It is possible that $\mathrm{N}$-formylation helps protect the peptides from aminopeptidases, similarly to other modifications of the N-terminus found in other RiPPs (e.g. acetyl, ${ }^{\mathbf{4 6 2}}$ lactyl, ${ }^{140,146}$ methyl, ${ }^{150,180,181,463}$ 2-oxobutyryl, ${ }^{163}$ and 2-oxopropionyl $^{464}$ ). We note that $N$-formylated peptides are not RiPPs since they are not post-translationally modified, but they are included here as they are often detected when searching for RiPPs by mass spectrometry.

\section{PQQ, pantocin, and thyroid hormones}

A number of other molecules that are smaller than the compounds discussed thus far are generated from a ribosomally synthesized peptide. Pyrroloquinoline quinone (PQQ, Fig. 32A) is a cofactor for several bacterial dehydrogenases. ${ }^{465} \mathrm{In}$ a series of studies in the late 1980s and early 1990s, PQQ was first shown to be generated from a ribosomally synthesized precursor peptide. ${ }^{\mathbf{4 6 6 - 4 6 8}}$ Currently, the biosynthetic genes have been found in over 100 bacteria. $^{8}$ The precursor peptide is PqqA, a 22 amino acid peptide containing a conserved glutamate and tyrosine that are oxidatively morphed into the final structure and excised from the peptide by a series of enzymatic reactions (Fig. 32A). ${ }^{\mathbf{4 6 6 , 4 6 9}}$ Genetic studies have implicated PqqACDE to be required for PQQ biosynthesis, ${ }^{\mathbf{8 , 4 7 0 , 4 7 1}}$ but the details of the PTM process are largely unknown. PqqC has been shown to catalyze the final oxidation step in an oxygen dependent mechanism. ${ }^{\mathbf{4 7}}$ PqqB and PqqE, a putative non-heme iron oxygenase and a radical-SAM protein respecutively, carry out currently unknown transformations. ${ }^{\mathbf{8} 47}$ PqqE and PqqD form a protein complex that may be important for the overall timing of the reactions and/or the protection of reactive intermediates. ${ }^{\mathbf{8 , 4 7}}$ Although PqqF is not absolutely required, its sequence places it in the zinc-dependent protease family and it may be involved in the peptide excision process. At present the role of the flanking amino acids in the PqqA precursor peptide is not known, but it is likely that they serve a similar function as the recognition sequences discussed for cyanobactin, amatoxin, and cyclotide biosynthesis. With only two, non-sequential amino acids of the precursor peptide ending up in the final product, the term core peptide is not pertinent. As mentioned before, PQQ is made by an overall process that is similar to the other RiPPs discussed here, but the final product is no longer a peptide.

Another potential small molecule excised from a larger protein is coelenterazine (also called coelenterate-type luciferin, Fig. 32B). This molecule is used as a light emitting substrate by various luciferases and $\mathrm{Ca}^{2+}$-binding photoproteins in marine organisms. It is not well defined what organisms produce coelenterazine and its biosynthesis is currently unresolved. However, two observations suggest it may be ribosomally synthesized. First, feeding experiments indicate the compound is made from L-Tyr and L-Phe. ${ }^{475}$ Second, and more intriguing, expression in $E$. coli of a mutant of the green fluorescent protein 
A
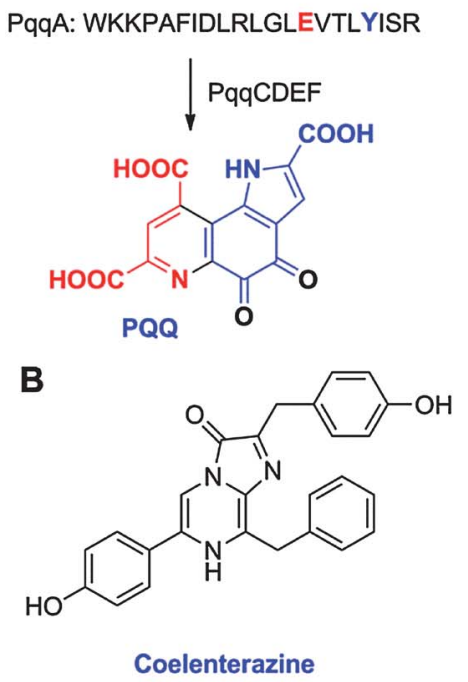

C PaaP: 11aa-SAITEENAMY-9 AA

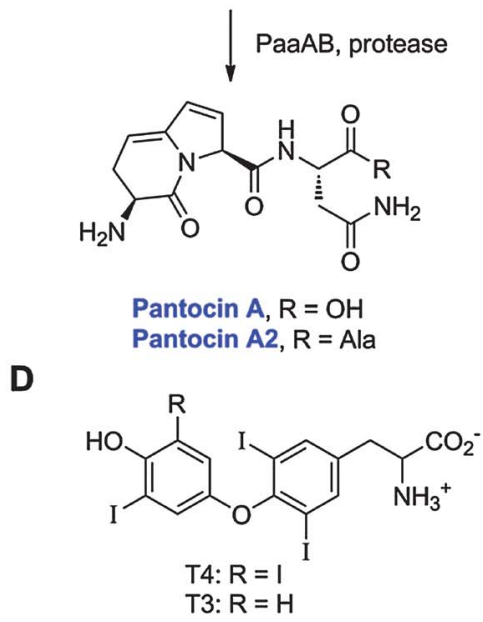

Fig. 32 Structures of (A) PQQ, (B) coelenterazine, (C) the pantocins, and (D) the thyroid hormones $\mathrm{T} 3$ and $\mathrm{T} 4$. For PQQ and the pantocins, the ribosomally synthesized precursor peptides from which they are produced are also depicted.

from Aequorea victoria in which the conserved sequence FSYG leading to the GFP chromophore was replaced with FYYG resulted in the production of coelenterazine ${ }^{476}$ At present, no details are available regarding this remarkable observation, but it suggests that coelenterazine can be made from a ribosomally synthesized protein.

Another example of a small molecule produced from a much larger ribosomally synthesized precursor is pantocin A produced by the bacterium Pantoea agglomerans (Fig. 32C). ${ }^{477}$ The gene paaP in its biosynthetic cluster encodes a 30-residue peptide with the sequence -Glu-Glu-Asn- near its center. ${ }^{478}$ Mutagenesis and heterologous expression studies demonstrated that the final product is formed from PaaP by two enzymes, PaaA and PaaB, and unknown proteases in the host. ${ }^{478}$ PaaA has significant sequence similarity to the ThiF/MoeB family of proteins that adenylate carboxyl groups, hinting that it may be involved in cyclization of the side chain of the first Glu with the amide nitrogen of the second Glu residue. PaaB has a domain with homology to the $\mathrm{Fe}(\mathrm{II})-\alpha$-ketoglutarate dependent family of enzymes, suggesting it may catalyze an oxidation step that will be required for formation of the final product. As with the PQQ pathway, the roles of the flanking residues in the precursor peptide is not clear but they may be recognition sequences for PaaA and PaaB, and like many leader peptides, they may keep the biosynthetic intermediates inactive.

Perhaps the most remarkable example of construction of a biologically active small molecule from a ribosomally synthesized precursor is the assembly mechanism of the thyroid hormones 3,3',5,5'-tetraiodothyronine (T4) and 3,30,5-triiodothyronine (T3) (Fig. 32D). These compounds are not made from free Tyr but rather from Tyr imbedded in thyroglobulin, a very large glycoprotein comprised of two identical subunits of $330000 \mathrm{Da}$ each. ${ }^{479}$ The aromatic rings of proximal Tyr residues are diiodinated by thyroid peroxidase, and this enzyme subsequently carries out the oxidatively coupling reaction of two iodinated Tyr residues. Hydrolysis then releases T4 and a small amount of T3 from incompletely iodinated Tyr. Since T3 is the major active form, T4 is converted to T3 by iodothyronine deiodinases. ${ }^{479}$ Although clearly this biosynthetic pathway is very different from that of the other compounds discussed in this review, the thyroid hormone examples provide yet another demonstration of the diverse ways that nature makes use of ribosomal products to generate small molecules.

\section{Shorthand linear notations for RiPPs}

As a consequence of their generally large molecular structures, shorthand nomenclature has been introduced for several of the subclasses of RiPPs (e.g. for lanthipeptides in Fig. 4). Here, we suggest a more general shorthand notation that can represent structures of RiPPs in linear format, which is useful for description of structures in abstracts of manuscripts and conference proceedings. The proposed notation uses a prefix code of 3-4 characters used to specify the type of modification for each modified amino acid, which are identified by their residue number in the core peptide sequence. For instance, 1$\mathrm{N} \alpha \mathrm{Ac}$ refers to acetylation of the $\mathrm{N}$-terminus of residue 1 (e.g. in microviridin B, Fig. 33), $22-\Delta \mathrm{CO}_{2}$ refers to decarboxylation of residue 22 (e.g. gallidermin, Fig. 33), and $1-\mathrm{C} \gamma \mathrm{OH}$ refers to hydroxylation of the $\gamma$-carbon of residue 1 (e.g. $\alpha$-amanitin, Fig. 34). For post-translational modifications that introduce crosslinks, the amino acids that are crosslinked are indicated first by residue number followed by the type of crosslink. For instance, $3 R-7-\mathrm{C} \beta S$ refers to a crosslink between the $\beta$-carbon of amino acid 3 and the sulfur of residue 7 , with the stereochemistry at $\mathrm{C} \beta$ being $R$ (e.g. the A-ring of nisin, Fig. 33), 1-8$\mathrm{N} \alpha \mathrm{C}$ refers to N-to-C cyclization between the N-terminal amino group of residue 1 and the C-terminal carboxylate of residue 8 (e.g. patellamide C, Fig. 33), 5-19-SS refers to a disulfide between residues 5 and 19 (e.g. kalata B1, Fig. 34), and 4-31-SC $\alpha$ refers to a crosslink between the sulfur of residue 4 to the $\alpha$-carbon of residue 31 (e.g. in subtilosin, Fig. 34). All modifications in a given peptide are provided in front of the linear sequence of the core peptide in square brackets starting with the lowest residue 


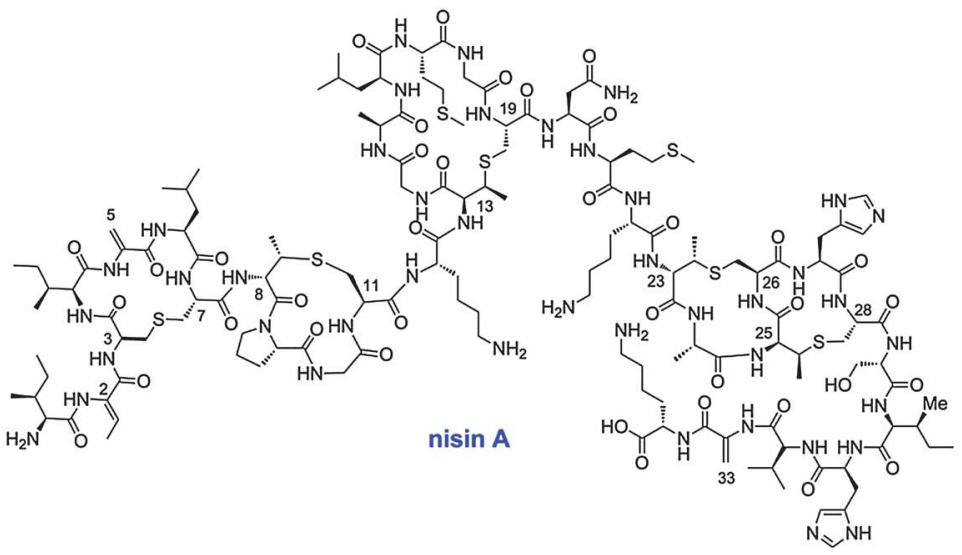

$[14,22-\triangle \alpha \beta],\left[22-\Delta \mathrm{CO}_{2}\right],[3-7,8-11,16-21,19-22-C \beta S]-I A A K F L C A b u P G C A K A b u G A F N A Y A C$
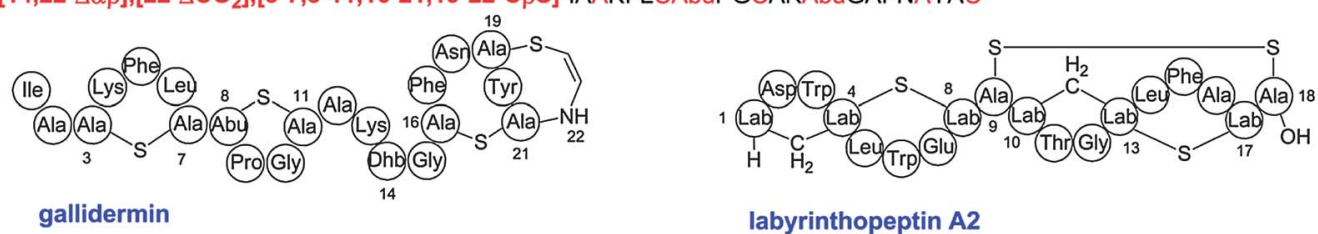

labyrinthopeptin A2

[1-4,10-13-C $\beta C \alpha$ ], [4-8,13-17-C $\beta$ S],[9-18-SS]-ADWALWECCATGALFACC

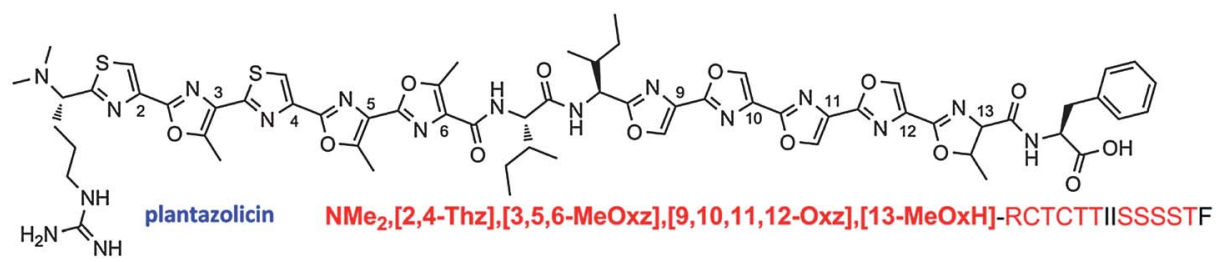

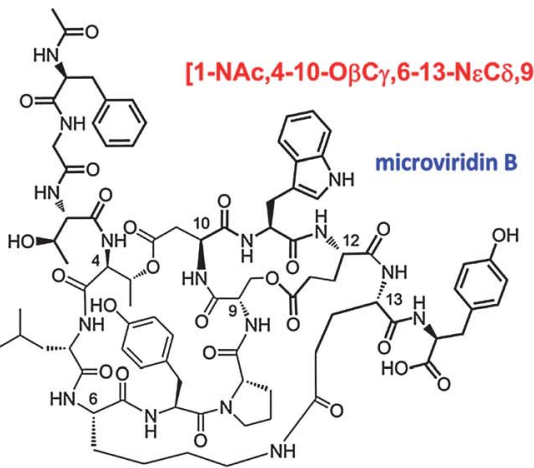

DWEEY

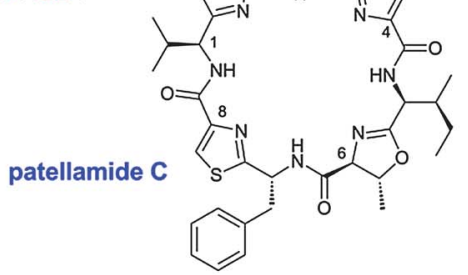

[1-8-N $\alpha$ C],[2,6-MeOxH],[4,8-Thz]-VTACITFC

Fig. 33 Select examples of the linear description of RiPPs. The linear description is shown above each chemical structure. The 3-4 character descriptors are shown in bold red font, the amino acids to which they refer are shown in non-bold red font. Abu, 2-aminobutyric acid.

numbers and working towards the higher residues. When multiple identical modifications are present, they are grouped together as illustrated for the five (methyl)lanthionine crosslinks in nisin (Fig. 33). The pre-fixes for a select number of PTMs discussed in this review are listed in Table 5, and examples of select RiPPs are shown in Fig. 33 and 34. Using the same reasoning, new pre-fixes can be readily introduced for the many PTMs that are not covered in Table 5.

It is likely that the future will see more and more engineered, non-natural RiPP analogs having one or more amino acid substitutions, insertions, or deletions. Such molecules should be denoted by standard mutagenesis nomenclature. Analogs containing additional modified residues by in vitro synthesis or by synthetic biology approaches can in most cases also be designated by the standard nomenclature, e.g. a Met residue that is replaced by a chlorinated Trp residue at postion 17 in nisin would be designated as Met17ClTrp. In cases where modifications from different RiPP classes are combined into one molecule (e.g. a classical lantibiotic attached to a lasso-peptide-like ring), the new molecule should get a descriptive name (e.g. nisin[1-18]-GSG linker-capistruin[1-9]), accompanied by a description of the structure at the amino acid level according to the proposed nomenclature rules described in this paper. Use of newly invented names, such as lassonisin or micronisin to describe hybrids are 
[1-35-N $\alpha$ C],[4-31, 7-28, 13-22SC $\alpha$ ]- NKGCATCSIGAACLVDGPIPDFEIAGATGLFGLWG

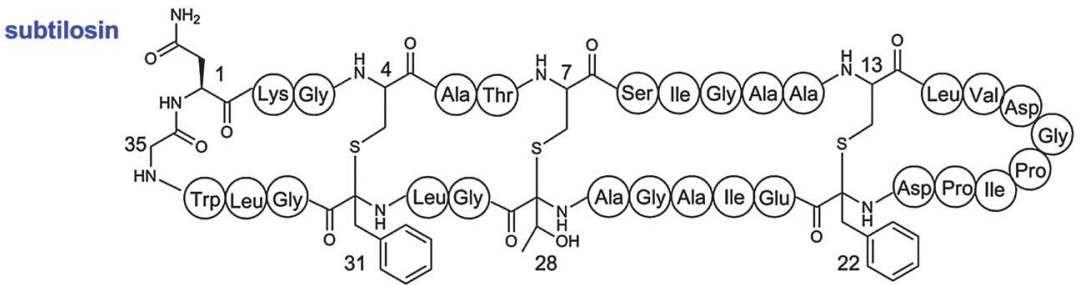

[1-9-N $\alpha$ C $\gamma$ @(10-19)]-GTPGFQTPDARVISRFGFN

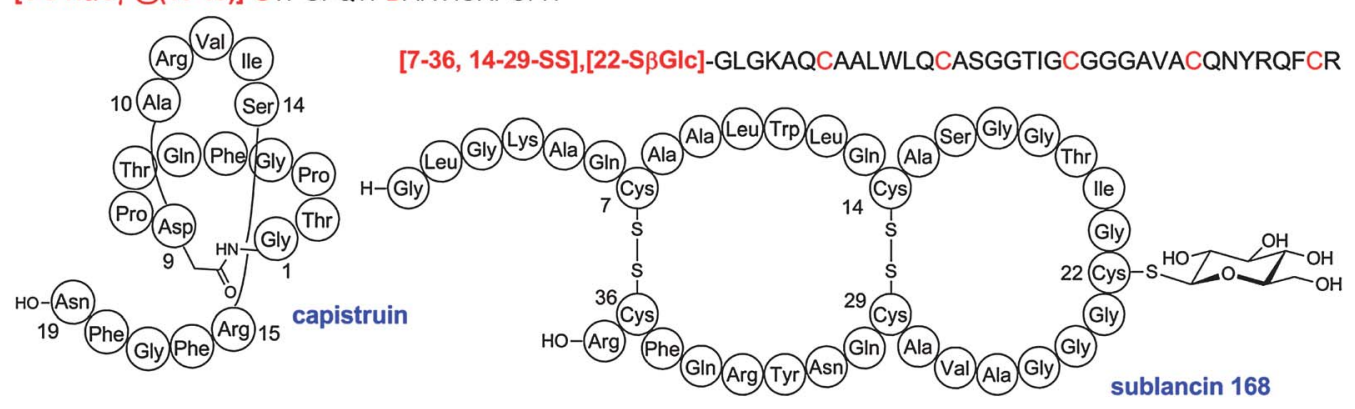

[1-29-NaC],[5-19,9-21,14-26-SS]-GLPVCGETCVGGTCNTPGCTCSWPVCTRN

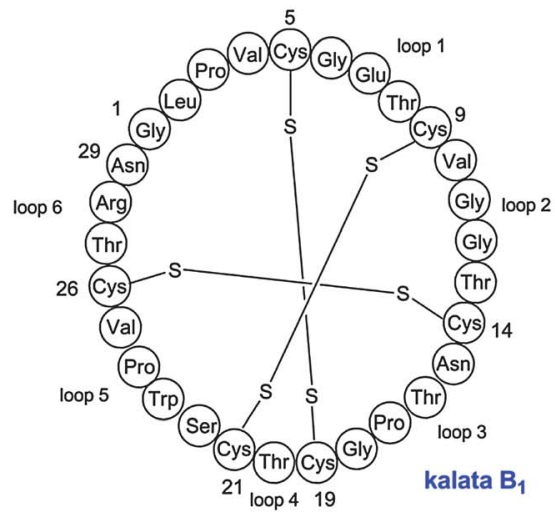

[1-8-N $\alpha \mathrm{C}],[1-\mathrm{C} \gamma \mathrm{OH}, \mathrm{C} \delta \mathrm{OH}],[2-6-\mathrm{C} 2 \mathrm{~S}(\mathrm{O})],[8-\mathrm{C} 3 \mathrm{OH}]-\mathrm{IWGIGCNP}$

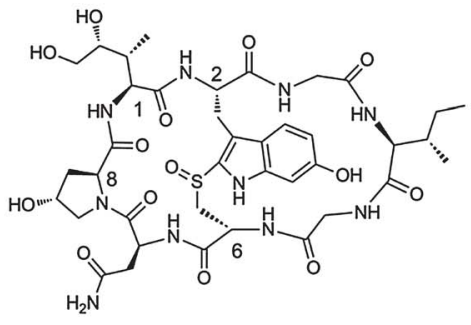

$\alpha$-amanitin

Fig. 34 Additional examples of linear shorthand notation for RiPPs.

discouraged as they would cause possible confusion, especially if many such analogs are prepared.

\section{Outlook}

The ribosomal origin of some of the classes of RiPPs discussed in this review has been known for quite some time, but for most classes this realization has been reached more recently as a result of the rapid advancement of DNA sequencing techniques. The availability of genome sequences first allowed the identification of putative precursor genes for known natural products. For compounds of bacterial, archaeal, and fungal sources, the convenient clustering of genes also provided immediate insights into the biosynthetic pathways, but deciphering biosynthetic pathways has been more challenging for compounds from higher organisms. In recent years, the use of genome information has been shifting slowly from identification of the genes for the biosynthesis of known compounds to genome mining for new compounds. ${ }^{1}$ RiPPs are particularly suited for genome mining because of the direct link between precursor gene and final product and because of the relatively short biosynthetic pathways, which lend them well for heterologous expression. This approach has allowed both isolation of new compounds from producing organisms, $6,26,47,48,64,133,134,144,179,190,192,308,327,480,481$ and heterologous production of compounds that are not produced under laboratory conditions by the organism containing the biosynthetic genes ("silent or cryptic clusters"). ${ }^{89}$ These studies either used genes encoding related precursor peptides as query for searching genomes, resulting in analogs of known compounds, or used the genes encoding key biosynthetic enzymes, often resulting in more distant analogs of known natural products.

Although they have been quite successful, these genome mining strategies currently have two disadvantages. Unlike phenotypic screens for discovery of new natural products with a known bioactivity, the genome mining approach typically does not provide any clues about the biological activities of the products. A second current shortcoming of the genome mining approach is that compound classes that fall into new structural scaffolds are difficult to discover because neither their biosynthetic genes nor their precursor genes are known. The large number of short open reading frames in genomes that are not annotated during genome sequencing exercises suggests that such classes may be quite 
Table 5 Designations for some of the post-translational modifications found in RiPPs that allow their description in linear format. Some examples are shown in Fig. 33 and 34. This list is not a comprehensive listing of all PTMs found in RiPPs

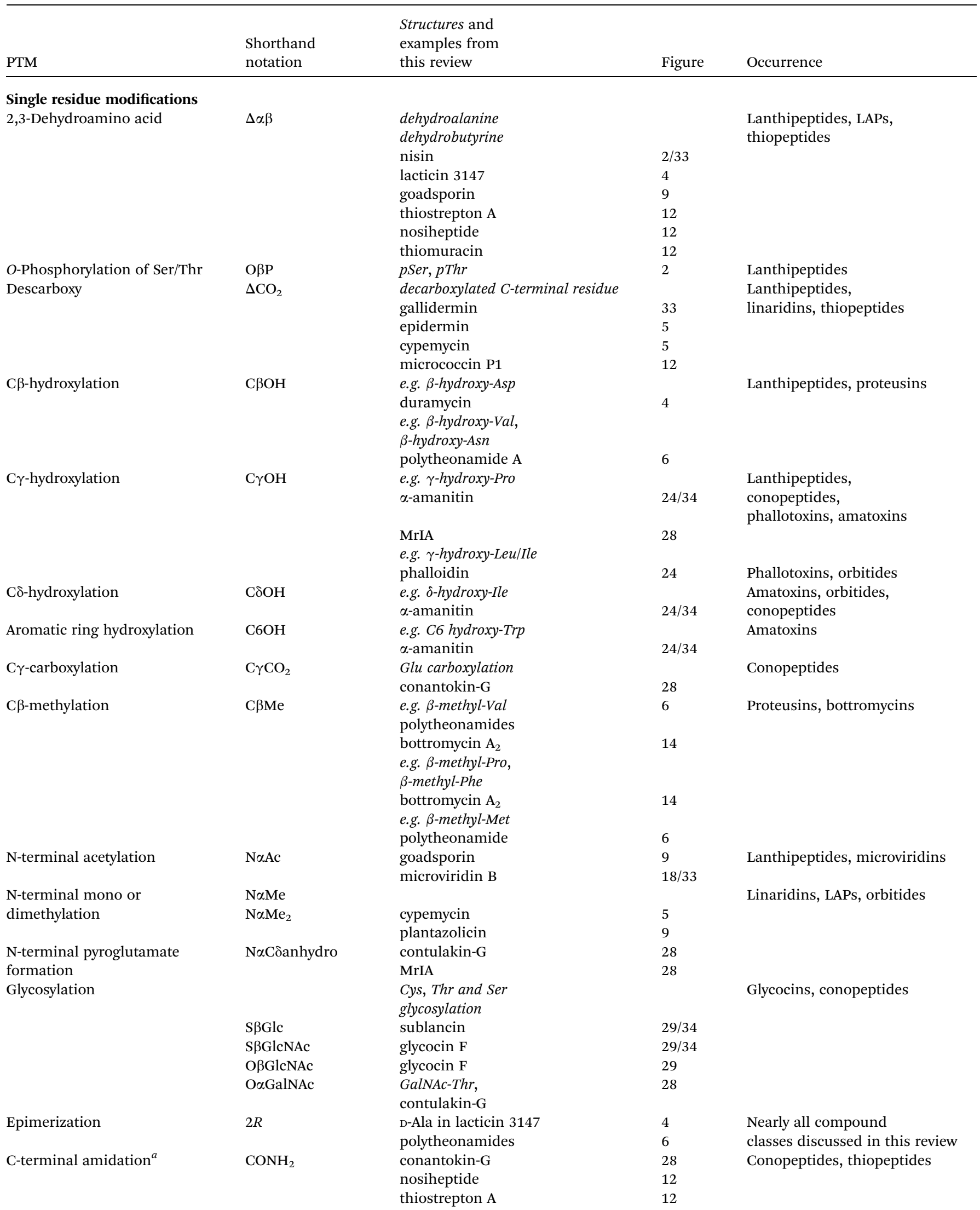


Table 5 (Contd.)

\begin{tabular}{|c|c|c|c|c|}
\hline PTM & $\begin{array}{l}\text { Shorthand } \\
\text { notation }\end{array}$ & $\begin{array}{l}\text { Structures and } \\
\text { examples from } \\
\text { this review }\end{array}$ & Figure & Occurrence \\
\hline \multicolumn{5}{|l|}{ Crosslink of two residues } \\
\hline \multirow[t]{7}{*}{ Disulfide } & \multirow[t]{7}{*}{ SS } & labyrinthopeptin & $4 / 33$ & \multirow{7}{*}{$\begin{array}{l}\text { Lanthipeptides, cyanobactins, } \\
\text { cyclotides, lasso peptides, } \\
\text { conopeptides, glycocins, } \\
\text { sactipeptides }\end{array}$} \\
\hline & & ulithiacyclamide & 10 & \\
\hline & & siamycins & 17 & \\
\hline & & kalata B1 & $25 / 34$ & \\
\hline & & MrIA & 28 & \\
\hline & & sublancin & $29 / 34$ & \\
\hline & & glycocin F & 29 & \\
\hline \multirow[t]{6}{*}{ Lanthionine } & \multirow[t]{6}{*}{$\mathrm{SC} \beta$ or $\mathrm{C} \beta \mathrm{S}^{b}$} & thioether & & \multirow[t]{6}{*}{ Lanthipeptides } \\
\hline & & nisin & 2 & \\
\hline & & actagardine & 4 & \\
\hline & & duramycin & 4 & \\
\hline & & lacticin 3147 & 4 & \\
\hline & & SapT & 4 & \\
\hline Lanthionine sulfoxide & $\mathrm{S}(\mathrm{O}) \mathrm{C} \beta$ & actagardine & 4 & Lanthipeptides \\
\hline \multirow[t]{2}{*}{ Lysinoalanine } & \multirow[t]{2}{*}{$N \varepsilon C \beta$} & secondary amine & & \multirow[t]{2}{*}{ Lanthipeptides } \\
\hline & & duramycin & 4 & \\
\hline \multirow[t]{2}{*}{$\alpha$-carbon to $\beta$-carbon } & \multirow[t]{2}{*}{$\mathrm{C} \alpha \mathrm{C} \beta$} & labionin & & \multirow[t]{2}{*}{ Lanthipeptides } \\
\hline & & labyrinthopeptin & $4 / 33$ & \\
\hline Oxazole & Oxz & microcin B17 & 9 & LAPs, cyanobactins, thiopeptides \\
\hline Oxazoline & $\mathrm{OxH}$ & patellamide A & 10 & LAPs, cyanobactins, thiopeptides \\
\hline \multirow[t]{3}{*}{ Methyloxazole } & \multirow[t]{3}{*}{ MeOxz } & plantazolicin & $9 / 33$ & \multirow[t]{3}{*}{ LAPs, cyanobactins } \\
\hline & & goadsporin & 9 & \\
\hline & & microcyclamide & 10 & \\
\hline Methyloxazoline & $\mathrm{MeOxH}$ & plantazolicin & $9 / 33$ & LAPs, cyanobactins \\
\hline & & patellamide A & 10 & \\
\hline & & patellamide $\mathrm{C}$ & 33 & \\
\hline Thiazole & Thz & microcin B17 & 9 & LAPs, cyanobactins, \\
\hline & & goadsporin & 9 & thiopeptides, bottromycins \\
\hline & & plantazolicin & $9 / 33$ & \\
\hline & & patellamide A & 10 & \\
\hline & & patellamide C & 33 & \\
\hline & & microcyclamide & 10 & \\
\hline & & trichamide & 10 & \\
\hline & & bottromycin $\mathrm{A}_{2}$ & 14 & \\
\hline Thiazoline & ThH & trunkamide & 10 & LAPs, cyanobactins, thiopeptides \\
\hline Tryptathionine & $\mathrm{C} 2 \mathrm{~S}$ or $\mathrm{SC} 2^{b}$ & thioether & & Phallotoxins \\
\hline & & phalloidin & 24 & \\
\hline Tryptathionine sulfoxide & $\mathrm{C} 2 \mathrm{~S}(\mathrm{O})$ & $\alpha$-amanitin & $24 / 34$ & Amatoxins \\
\hline$\alpha$-carbon linked to sulfur & $\mathrm{SC} \alpha(\text { or } \mathrm{C} \alpha \mathrm{S})^{b}$ & thioether & & Sactipeptides, thiopeptides \\
\hline & & subtilosin & $20 / 34$ & \\
\hline & & thuricin CD & 20 & \\
\hline C- to N-terminal cyclization & $\mathrm{N} \alpha \mathrm{C}$ & patellamide A & 10 & Cyanobactins, amanitins, \\
\hline & & patellamide C & 33 & cyclotides, orbitides, sactipeptides \\
\hline & & subtilosin & $20 / 34$ & \\
\hline & & enterocin AS-48 & 22 & \\
\hline & & carnocyclin A & 22 & \\
\hline & & $\alpha$-amanitin & $24 / 34$ & \\
\hline & & kalata B1 & 34 & \\
\hline & & cyclolinopeptides & 27 & \\
\hline & & segetalins & 26 & \\
\hline N-terminal amine & $\mathrm{N} \alpha \mathrm{C} \delta$ & isopeptide & & \\
\hline and Glu/Asp side chain & $\mathrm{N} \alpha \mathrm{C} \gamma$ & & & \\
\hline $\mathrm{N}$-terminal amine and & 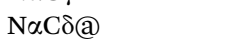 & siamycin & 17 & Lasso peptides \\
\hline Glu/Asp side chain & NaCr@ & capistruin & $17 / 34$ & \\
\hline $\begin{array}{l}\text { peptide tail goes through } \\
\text { the lactam }\end{array}$ & & microcin J25 & 16 & \\
\hline Indication of size of & $\mathrm{N} \alpha \mathrm{C} \delta-@[9-21]$ & microcin J25 & 17 & Lasso peptides \\
\hline peptide tail through lactam & $\mathrm{N} \alpha \mathrm{C} \gamma-@[10-19]$ & capistruin & 34 & \\
\hline Lys side chain with & $\mathrm{C} \delta_{\mathrm{N}} \varepsilon$ or $\mathrm{N} \varepsilon \mathrm{C} \delta^{b}$ & isopeptide & & Microviridins \\
\hline Glu side chain & & microviridin $\mathrm{B}$ & $18 / 33$ & \\
\hline
\end{tabular}




\begin{tabular}{|c|c|c|c|c|}
\hline PTM & $\begin{array}{l}\text { Shorthand } \\
\text { notation }\end{array}$ & $\begin{array}{l}\text { Structures and } \\
\text { examples from } \\
\text { this review }\end{array}$ & Figure & Occurrence \\
\hline $\begin{array}{l}\text { Lys side chain with } \\
\text { Asp side chain }\end{array}$ & $\mathrm{N} \varepsilon \mathrm{C} \gamma$ or $\mathrm{C} \gamma \mathrm{N} \varepsilon^{b}$ & isopeptide & & Microviridins \\
\hline Asp side chain with Ser/Thr: & $\mathrm{C} \gamma \mathrm{O} \beta$ or $\mathrm{O} \beta \mathrm{C} \gamma^{b}$ & $\begin{array}{l}\text { ester } \\
\text { microviridin B } \\
\text { marinostatin 1-12 }\end{array}$ & $\begin{array}{l}18 / 33 \\
18\end{array}$ & Microviridins \\
\hline Glu side chain with Ser/Thr: & $\mathrm{C} \delta \mathrm{O} \beta$ or $\mathrm{O} \beta \mathrm{C} \delta^{b}$ & $\begin{array}{l}\text { ester } \\
\text { microviridin B }\end{array}$ & $18 / 33$ & Microviridins \\
\hline $\begin{array}{l}\text { Cys side chain and } \\
\text { C-terminal carboxylate }\end{array}$ & $\mathrm{S} \beta \mathrm{CO}$ & $\begin{array}{l}\text { thioester } \\
\text { AIP-I }\end{array}$ & 30 & AIPs \\
\hline
\end{tabular}

common. Even with these current limitations, genome mining is likely to become an even more active area of research that potentially may uncover compounds with exciting bioactivities because analysis of the currently available genomes for known classes illustrates the ubiquitous nature of RiPPs. Going forward, it will be important to use the most powerful bioinformatics methods to analyze the explosion of sequence information. In this respect, Hidden Markov Models (HMMs) can identify distantly related protein families when other methods do not. ${ }^{\mathbf{8 2 - 4 8 4}}$ The recently developed, freely available genome mining tool Bagel2 (http:// bagel2.molgenrug.nl/) also offers many features to screen for putative (modified) antimicrobial peptides based on a large set of search criteria. ${ }^{455}$ Another useful site in the context of posttranslational modifications is the RESID Database of Protein Modifications (http://www.ebi.ac.uk/RESID/index.html), ${ }^{486}$ which provides a comprehensive compilation of naturally occurring modifications annotated in the UniProt Protein Knowledgebase.

Caution is warranted with respect to drawing conclusions from bioinformatics alone as several common expectations about RiPP biosynthesis systems are not completely reliable. In particular, sequence similarity of precursor peptides does not guarantee that the final products belong to the same family of RiPPs. For example, lanthionine synthetases, cyclodehydratases, and radical SAM enzymes all act on members of the nitrile hydratase-like leader peptide family, as well as the nif11-like leader peptide family., ${ }^{\mathbf{3 , 4 6 5}}$ Meanwhile, proximity between genes for a RiPP precursor and a maturase does not guarantee a target/substrate relationship. The genes encoding the precursor peptide, its maturation enzyme(s), and its export transporter can all be far apart in a genome. ${ }^{26,487}$

This review has attempted to bring together groups of scientists working on different classes of RiPPs to work out a common nomenclature within the class and across classes. We hope that similar concerted efforts will be used to classify new RiPP classes and their biosynthetic machinery that are yet to be discovered. In particular, we recommend in general that the gene encoding the precursor gene is always given the designation A. If surrounding genes have homology with enzymes used by the RiPP classes discussed in this review, we recommend trying to use a similar gene designation as that used previously to illustrate the connection. For all genes encoding proteins with unknown function, we recommend alphabetically naming the genes in the order they appear in the operon in the first disclosure of the gene cluster. All studies following the initial report of a new RiPP are strongly encouraged to use the same letter designations of the initial study such that a common nomenclature arises.

\section{Acknowledgements}

The recommendations in this review were compiled from the answers to two sets of successive questionnaires. Several discussion groups then made recommendations to the entire group of authors. The discussion groups were led by David Craik (cyclotides), Elke Dittmann (microviridins), Baldomero Olivera (conopeptides), Sylvie Rebuffat (microcins), Eric Schmidt (cyanobactins and LAPs), Wilfred van der Donk (lanthipeptides and glycocins), John Vederas (sactipeptides and N-to-C cyclized peptides), and Christopher Walsh (thiopeptides). The final write-ups for the several classes of compounds were provided by David Craik (cyclotides), Elke Dittmann (microviridins), Wendy Kelly (thiopeptides), Douglas Mitchell (LAPs), Joern Piel (proteusins), Baldomero Olivera (conopeptides), Martin Reaney (orbitides), Sylvie Rebuffat (microcins and lasso peptides), Eric Schmidt (cyanobactins), Wilfred van der Donk (introduction, lanthipeptides, linaridins, bottromycins, glycocins, quorum sensing peptides, PQQ, methanobactin, linear nomenclature, and outlook) and John Vederas (sactipeptides, N-to-C cyclized peptides, linear nomenclature). Coordination of the discussions and assembly and editing of the final review was done by Wilfred van der Donk. We thank Dr Qi Zhang (UIUC) for help with illustrations.

\section{References}

1 J. E. Velásquez and W. A. van der Donk, Curr. Opin. Chem. Biol., 2011, 15, 11.

2 H. Wang, D. P. Fewer and K. Sivonen, PLoS One, 2011, 6, e22384. 
3 D. H. Haft, M. K. Basu and D. A. Mitchell, BMC Biol., 2010, 8, 70.

4 K. Murphy, O. O’Sullivan, M. C. Rea, P. D. Cotter, R. P. Ross and C. Hill, PLoS One, 2011, 6, e20852.

5 D. H. Haft and M. K. Basu, J. Bacteriol., 2011, 193, 2745.

6 R. D. Kersten, Y.-L. Yang, Y. Xu, P. Cimermancic, S.-J. Nam, W. Fenical, M. A. Fischbach, B. S. Moore and P. C. Dorrestein, Nat. Chem. Biol., 2011, 7, 794.

7 S. W. Lee, D. A. Mitchell, A. L. Markley, M. E. Hensler, D. Gonzalez, A. Wohlrab, P. C. Dorrestein, V. Nizet and J. E. Dixon, Proc. Natl. Acad. Sci. U. S. A., 2008, 105, 5879.

8 Y. Q. Shen, F. Bonnot, E. M. Imsand, J. M. Rosefigura, K. Sjölander and J. P. Klinman, Biochemistry, 2012, 51, 2265.

9 Q. Qin, E. J. McCallum, Q. Kaas, J. Suda, I. Saska, D. J. Craik and J. S. Mylne, BMC Genomics, 2010, 11, 111.

10 A. O. Lluisma, B. A. Milash, B. Moore, B. M. Olivera and P. Bandyopadhyay, Marine Genomics, 2012, 43.

11 J. A. McIntosh, M. S. Donia and E. W. Schmidt, Nat. Prod. Rep., 2009, 26, 537.

12 S. Duquesne, D. Destoumieux-Garzon, J. Peduzzi and S. Rebuffat, Nat. Prod. Rep., 2007, 24, 708.

13 T. R. Klaenhammer, FEMS Microbiol. Rev., 1993, 12, 39.

14 R. Finking and M. A. Marahiel, Annu. Rev. Microbiol., 2004, $58,453$.

15 M. A. Fischbach and C. T. Walsh, Chem. Rev., 2006, 106, 3468.

16 T. J. Oman and W. A. van der Donk, Nat. Chem. Biol., 2010, 6, 9.

17 L. Huo, S. Rachid, M. Stadler, S. C. Wenzel and R. Müller, Chem. Biol., 2012, 19, 1278.

18 W. J. K. Crone, F. J. Leeper and A. W. Truman, Chem. Sci., 2012, 3, 3516.

19 J. P. Gomez-Escribano, L. Song, M. J. Bibb and G. L. Challis, Chem. Sci., 2012, 3, 3522.

20 Y. Hou, M. D. Tianero, J. C. Kwan, T. P. Wyche, C. R. Michel, G. A. Ellis, E. Vazquez-Rivera, D. R. Braun, W. E. Rose, E. W. Schmidt and T. S. Bugni, Org. Lett., 2012, 14, 5050.

21 E. W. Schmidt, J. T. Nelson, D. A. Rasko, S. Sudek, J. A. Eisen, M. G. Haygood and J. Ravel, Proc. Natl. Acad. Sci. U. S. A., 2005, 102, 7315.

22 H. E. Hallen, H. Luo, J. S. Scott-Craig and J. D. Walton, Proc. Natl. Acad. Sci. U. S. A., 2007, 104, 19097.

23 M. Trabi, J. S. Mylne, L. Sando and D. J. Craik, Org. Biomol. Chem., 2009, 7, 2378.

24 S. R. Woodward, L. J. Cruz, B. M. Olivera and D. R. Hillyard, EMBO J., 1990, 9, 1015.

25 M. S. Donia, B. J. Hathaway, S. Sudek, M. G. Haygood, M. J. Rosovitz, J. Ravel and E. W. Schmidt, Nat. Chem. Biol., 2006, 2, 729.

26 B. Li, D. Sher, L. Kelly, Y. Shi, K. Huang, P. J. Knerr, I. Joewono, D. Rusch, S. W. Chisholm and W. A. van der Donk, Proc. Natl. Acad. Sci. U. S. A., 2010, 107, 10430.

27 O. P. Kuipers, G. Bierbaum, B. Ottenwälder, H. M. Dodd, N. Horn, J. Metzger, T. Kupke, V. Gnau, R. Bongers, P. van den Bogaard, H. Kosters, H. S. Rollema, W. M. de Vos, R. J. Siezen, G. Jung, F. Götz, H. G. Sahl and M. J. Gasson, Antonie van Leeuwenhoek, 1996, 69, 161.
28 P. D. Cotter, L. H. Deegan, E. M. Lawton, L. A. Draper, P. M. O'Connor, C. Hill and R. P. Ross, Mol. Microbiol., 2006, 62, 735.

29 C. Chatterjee, G. C. Patton, L. Cooper, M. Paul and W. A. van der Donk, Chem. Biol., 2006, 13, 1109.

30 R. Rink, J. Wierenga, A. Kuipers, L. D. Kluskens, A. J. M. Driessen, O. P. Kuipers and G. N. Moll, Appl. Environ. Microbiol., 2007, 73, 5809.

31 O. Pavlova, J. Mukhopadhyay, E. Sineva, R. H. Ebright and K. Severinov, J. Biol. Chem., 2008, 283, 25589.

32 M. S. Donia, J. Ravel and E. W. Schmidt, Nat. Chem. Biol., 2008, 4, 341.

33 A. N. Appleyard, S. Choi, D. M. Read, A. Lightfoot, S. Boakes, A. Hoffmann, I. Chopra, G. Bierbaum, B. A. Rudd, M. J. Dawson and J. Cortés, Chem. Biol., 2009, 16, 490.

34 M. R. Levengood, P. J. Knerr, T. J. Oman and W. A. van der Donk, J. Am. Chem. Soc., 2009, 131, 12024.

35 M. R. Islam, K. Shioya, J. Nagao, M. Nishie, H. Jikuya, T. Zendo, J. Nakayama and K. Sonomoto, Mol. Microbiol., 2009, 72, 1438.

36 M. G. Acker, A. A. Bowers and C. T. Walsh, J. Am. Chem. Soc., 2009, 131, 17563.

37 D. A. Mitchell, S. W. Lee, M. A. Pence, A. L. Markley, J. D. Limm, V. Nizet and J. E. Dixon, J. Biol. Chem., 2009, 284, 13004.

38 A. A. Bowers, M. G. Acker, A. Koglin and C. T. Walsh, J. Am. Chem. Soc., 2010, 132, 7519.

39 S. J. Pan and A. J. Link, J. Am. Chem. Soc., 2011, 133, 5016.

40 T. A. Knappe, F. Manzenrieder, C. Mas-Moruno, U. Linne, F. Sasse, H. Kessler, X. Xie and M. A. Marahiel, Angew. Chem., Int. Ed., 2011, 50, 8714.

41 T. A. Knappe, U. Linne, L. Robbel and M. A. Marahiel, Chem. Biol., 2009, 16, 1290.

42 C. Li, F. Zhang and W. L. Kelly, Chem. Commun., 2012, 48, 558.

43 C. Li, F. Zhang and W. L. Kelly, Mol. BioSyst., 2011, 7, 82.

44 H. Onaka, M. Nakaho, K. Hayashi, Y. Igarashi and T. Furumai, Microbiology, 2005, 151, 3923.

45 M. F. Freeman, C. Gurgui, M. J. Helf, B. I. Morinaka, A. R. Uria, N. J. Oldham, H. G. Sahl, S. Matsunaga and J. Piel, Science, 2012, 338, 387.

46 W. L. Kelly, L. Pan and C. Li, J. Am. Chem. Soc., 2009, 131, 4327.

47 L. C. Wieland Brown, M. G. Acker, J. Clardy, C. T. Walsh and M. A. Fischbach, Proc. Natl. Acad. Sci. U. S. A., 2009, 106, 2549.

48 R. Liao, L. Duan, C. Lei, H. Pan, Y. Ding, Q. Zhang, D. Chen, B. Shen, Y. Yu and W. Liu, Chem. Biol., 2009, 16, 141.

49 R. P. Morris, J. A. Leeds, H. U. Naegeli, L. Oberer, K. Memmert, E. Weber, M. J. Lamarche, C. N. Parker, N. Burrer, S. Esterow, A. E. Hein, E. K. Schmitt and P. Krastel, J. Am. Chem. Soc., 2009, 131, 5946.

50 C. T. Walsh, S. J. Malcolmson and T. S. Young, ACS Chem. Biol., 2012, 7, 429.

51 Y. M. Li, J. C. Milne, L. L. Madison, R. Kolter and C. T. Walsh, Science, 1996, 274, 1188. 
52 J. O. Melby, N. J. Nard and D. A. Mitchell, Curr. Opin. Chem. Biol., 2011, 15, 369.

53 J. Lee, J. McIntosh, B. J. Hathaway and E. W. Schmidt, J. Am. Chem. Soc., 2009, 131, 2122.

54 J. D. Walton, H. E. Hallen-Adams and H. Luo, Biopolymers, 2010, 94, 659.

55 M. J. van Belkum, L. A. Martin-Visscher and J. C. Vederas, Trends Microbiol., 2011, 19, 411.

56 S. Gunasekera, N. L. Daly, M. A. Anderson and D. J. Craik, IUBMB Life, 2006, 58, 515.

57 P. J. Knerr and W. A. van der Donk, Annu. Rev. Biochem., 2012, 81, 479.

58 R. S. Roy, S. Kim, J. D. Baleja and C. T. Walsh, Chem. Biol., 1998, 5, 217.

59 G. C. Patton, M. Paul, L. E. Cooper, C. Chatterjee and W. A. van der Donk, Biochemistry, 2008, 47, 7342.

$60 \mathrm{~W}$. E. Houssen, S. H. Wright, A. P. Kalverda, G. S. Thompson, S. M. Kelly and M. Jaspars, ChemBioChem, 2011, 11, 1867.

61 T. Sprules, K. E. Kawulka, A. C. Gibbs, D. S. Wishart and J. C. Vederas, Eur. J. Biochem., 2004, 271, 1748.

62 J. L. Dutton, R. F. Renda, C. Waine, R. J. Clark, N. L. Daly, C. V. Jennings, M. A. Anderson and D. J. Craik, J. Biol. Chem., 2004, 279, 46858.

63 W. M. Müller, P. Ensle, B. Krawczyk and R. D. Süssmuth, Biochemistry, 2011, 50, 8362.

64 M. S. Donia and E. W. Schmidt, Chem. Biol., 2011, 18, 508.

65 Y. Kotake, S. Ishii, T. Yano, Y. Katsuoka and H. Hayashi, Biochemistry, 2008, 47, 2531.

66 S. Ishii, T. Yano, A. Ebihara, A. Okamoto, M. Manzoku and H. Hayashi, J. Biol. Chem., 2010, 285, 10777.

67 L. A. Furgerson Ihnken, C. Chatterjee and W. A. van der Donk, Biochemistry, 2008, 47, 7352.

68 W. L. Cheung, S. J. Pan and A. J. Link, J. Am. Chem. Soc., 2010, 132, 2514.

69 F. Garcia-Olmedo, A. Molina, J. M. Alamillo and P. Rodriguez-Palenzuela, Biopolymers, 1998, 47, 479.

70 R. E. Hancock and G. Diamond, Trends Microbiol., 2000, 8, 402.

71 L. Padovan, M. Scocchi and A. Tossi, Curr. Protein Pept. Sci., 2010, 11, 210.

72 A. A. Vassilevski, S. A. Kozlov and E. V. Grishin, Biochemistry (Moscow), 2009, 74, 1505.

73 S. Dutertre and R. J. Lewis, J. Biol. Chem., 2010, 285, 13315.

74 R. M. Kini and R. Doley, Toxicon, 2010, 56, 855.

75 D. C. Koh, A. Armugam and K. Jeyaseelan, Cell. Mol. Life Sci., 2006, 63, 3030.

76 L. A. Rogers, J. Bacteriol., 1928, 16, 321.

77 E. Gross and J. L. Morell, J. Am. Chem. Soc., 1971, 93, 4634.

78 N. Schnell, K.-D. Entian, U. Schneider, F. Götz, H. Zahner, R. Kellner and G. Jung, Nature, 1988, 333, 276.

79 L. Ingram, Biochim. Biophys. Acta, 1970, 224, 263.

80 G. W. Buchman, S. Banerjee and J. N. Hansen, J. Biol. Chem., 1988, 263, 16260.

81 C. Kaletta and K. D. Entian, J. Bacteriol., 1989, 171, 1597.

82 S. Banerjee and J. N. Hansen, J. Biol. Chem., 1988, 262, 9508.
83 C. Kaletta, K. D. Entian, R. Kellner, G. Jung, M. Reis and H. G. Sahl, Arch. Microbiol., 1989, 152, 16.

84 S. Boakes, J. Cortés, A. N. Appleyard, B. A. Rudd and M. J. Dawson, Mol. Microbiol., 2009, 72, 1126.

85 G. Bierbaum, H. Brötz, K. P. Koller and H. G. Sahl, FEMS Microbiol. Lett., 1995, 127, 121.

86 C. Kaletta, K. D. Entian and G. Jung, Eur. J. Biochem., 1991, 199, 411.

87 C. Chatterjee, L. M. Miller, Y. L. Leung, L. Xie, M. Yi, N. L. Kelleher and W. A. van der Donk, J. Am. Chem. Soc., 2005, 127, 15332.

88 W. M. Müller, T. Schmiederer, P. Ensle and R. D. Süssmuth, Angew. Chem., Int. Ed., 2010, 49, 2436.

89 Y. Goto, B. Li, J. Claesen, Y. Shi, M. J. Bibb and W. A. van der Donk, PLoS Biol., 2010, 8, e1000339.

90 A. K. Sen, A. Narbad, N. Horn, H. M. Dodd, A. J. Parr, I. Colquhoun and M. J. Gasson, Eur. J. Biochem., 1999, 261, 524 .

91 O. Koponen, M. Tolonen, M. Qiao, G. Wahlstrom, J. Helin and P. E. J. Saris, Microbiology, 2002, 148, 3561.

92 L. D. Kluskens, A. Kuipers, R. Rink, E. de Boef, S. Fekken, A. J. Driessen, O. P. Kuipers and G. N. Moll, Biochemistry, 2005, 44, 12827.

93 H. P. Weil, A. G. Beck-Sickinger, J. Metzger, S. Stevanovic, G. Jung, M. Josten and H. G. Sahl, Eur. J. Biochem., 1990, 194, 217.

94 C. Meyer, G. Bierbaum, C. Heidrich, M. Reis, J. Süling, M. I. Iglesias-Wind, C. Kempter, E. Molitor and H.-G. Sahl, Eur. J. Biochem., 1995, 232, 478.

95 B. Li, J. P. Yu, J. S. Brunzelle, G. N. Moll, W. A. van der Donk and S. K. Nair, Science, 2006, 311, 1464.

96 M. Helfrich, K. D. Entian and T. Stein, Biochemistry, 2007, 46, 3224.

97 K. Siegers, S. Heinzmann and K.-D. Entian, J. Biol. Chem., 1996, 271, 12294.

98 R. J. Siezen, O. P. Kuipers and W. M. de Vos, Antonie van Leeuwenhoek, 1996, 69, 171.

99 L. Xie, L. M. Miller, C. Chatterjee, O. Averin, N. L. Kelleher and W. A. van der Donk, Science, 2004, 303, 679.

100 Y. Goto, A. Ökesli and W. A. van der Donk, Biochemistry, 2011, 50, 891.

101 S. Kodani, M. E. Hudson, M. C. Durrant, M. J. Buttner, J. R. Nodwell and J. M. Willey, Proc. Natl. Acad. Sci. U. S. A., 2004, 101, 11448.

102 N. M. Okeley, M. Paul, J. P. Stasser, N. Blackburn and W. A. van der Donk, Biochemistry, 2003, 42, 13613.

103 K. Meindl, T. Schmiederer, K. Schneider, A. Reicke, D. Butz, S. Keller, H. Guhring, L. Vertesy, J. Wink, H. Hoffmann, M. Bronstrup, G. M. Sheldrick and R. D. Süssmuth, Angew. Chem., Int. Ed., 2010, 49, 1151.

104 H. Wang and W. A. van der Donk, ACS Chem. Biol., 2012, 7, 1529.

105 O. P. Kuipers, H. S. Rollema, W. M. Yap, H. J. Boot, R. J. Siezen and W. M. de Vos, J. Biol. Chem., 1992, 267, 24340.

106 W. Liu and J. N. Hansen, J. Biol. Chem., 1992, 267, 25078. 
107 H. S. Rollema, O. P. Kuipers, P. Both, W. M. de Vos and R. J. Siezen, Appl. Environ. Microbiol., 1995, 61, 2873.

108 G. Bierbaum, C. Szekat, M. Josten, C. Heidrich, C. Kempter, G. Jung and H. G. Sahl, Appl. Environ. Microbiol., 1996, 62, 385.

109 P. Chen, J. Novak, M. Kirk, S. Barnes, F. Qi and P. W. Caufield, Appl. Environ. Microbiol., 1998, 64, 2335.

110 Y. Shi, X. Yang, N. Garg and W. A. van der Donk, J. Am. Chem. Soc., 2011, 133, 2338.

111 F. Oldach, R. Al Toma, A. Kuthning, T. Caetano, S. Mendo, N. Budisa and R. D. Süssmuth, Angew. Chem., Int. Ed., 2012, $51,415$.

112 A. Chakicherla and J. N. Hansen, J. Biol. Chem., 1995, 270, 23533.

113 O. P. Kuipers, H. S. Rollema, W. M. de Vos and R. J. Siezen, FEBS Lett., 1993, 330, 23.

114 J. R. van der Meer, H. S. Rollema, R. J. Siezen, M. M. Beerthuyzen, O. P. Kuipers and W. M. de Vos, J. Biol. Chem., 1994, 269, 3555.

115 T. Stein and K. D. Entian, Rapid Commun. Mass Spectrom., 2002, 16, 103.

116 T. J. Oman, P. J. Knerr, N. A. Bindman, J. E. Velasquez and W. A. van der Donk, J. Am. Chem. Soc., 2012, 134, 6952.

117 T. Kupke, C. Kempter, G. Jung and F. Götz, J. Biol. Chem., 1995, 270, 11282.

118 A. Ökesli, L. E. Cooper, E. J. Fogle and W. A. van der Donk, J. Am. Chem. Soc., 2011, 133, 13753.

119 Y. Shi, A. Bueno and W. A. van der Donk, Chem. Commun., 2012, 48, 10966.

120 R. Rink, A. Kuipers, E. de Boef, K. J. Leenhouts, A. J. Driessen, G. N. Moll and O. P. Kuipers, Biochemistry, 2005, 44, 8873.

121 R. Rink, J. Wierenga, A. Kuipers, L. D. Kluskens, A. J. M. Driessen, O. P. Kuipers and G. N. Moll, Appl. Environ. Microbiol., 2007, 73, 1792.

122 M. R. Levengood and W. A. van der Donk, Bioorg. Med. Chem. Lett., 2008, 18, 3025.

123 G. N. Moll, A. Kuipers and R. Rink, Antonie van Leeuwenhoek, 2010, 97, 319.

124 M. R. Levengood, G. C. Patton and W. A. van der Donk, J. Am. Chem. Soc., 2007, 129, 10314.

125 R. Khusainov and O. P. Kuipers, ChemBioChem, 2012, 13, 2433.

126 S. Kodani, M. A. Lodato, M. C. Durrant, F. Picart and J. M. Willey, Mol. Microbiol., 2005, 58, 1368.

127 J. Lubelski, R. Rink, R. Khusainov, G. N. Moll and O. P. Kuipers, Cell. Mol. Life Sci., 2008, 65, 455.

128 G. Bierbaum and H. G. Sahl, Curr. Pharm. Biotechnol., 2009, 10, 2.

129 E. Breukink and B. de Kruijff, Nat. Rev. Drug Discovery, 2006, 5, 321.

130 A. M. Jones and J. M. Helm, Drugs, 2009, 69, 1903.

131 M. P. Ryan, R. W. Jack, M. Josten, H. G. Sahl, G. Jung, R. P. Ross and C. Hill, J. Biol. Chem., 1999, 274, 37544.

132 N. I. Martin, T. Sprules, M. R. Carpenter, P. D. Cotter, C. Hill, R. P. Ross and J. C. Vederas, Biochemistry, 2004, 43, 3049.
133 A. L. McClerren, L. E. Cooper, C. Quan, P. M. Thomas, N. L. Kelleher and W. A. van der Donk, Proc. Natl. Acad. Sci. U. S. A., 2006, 103, 17243.

134 E. M. Lawton, P. D. Cotter, C. Hill and R. P. Ross, FEMS Microbiol. Lett., 2007, 267, 64.

135 I. Wiedemann, T. Bottiger, R. R. Bonelli, A. Wiese, S. O. Hagge, T. Gutsmann, U. Seydel, L. Deegan, C. Hill, P. Ross and H. G. Sahl, Mol. Microbiol., 2006, 61, 285.

136 T. J. Oman, T. J. Lupoli, T. S. Wang, D. Kahne, S. Walker and W. A. van der Donk, J. Am. Chem. Soc., 2011, 133, 17544.

137 S. M. Morgan, M. P. O'Connor, P. D. Cotter, R. P. Ross and C. Hill, Antimicrob. Agents Chemother., 2005, 49, 2606.

138 J. M. Willey and W. A. van der Donk, Annu. Rev. Microbiol., 2007, 61, 477.

139 J. M. Willey, A. Willems, S. Kodani and J. R. Nodwell, Mol. Microbiol., 2006, 59, 731.

140 J. E. Velásquez, X. Zhang and W. A. van der Donk, Chem. Biol., 2011, 18, 857.

141 M. Begley, P. D. Cotter, C. Hill and R. P. Ross, Appl. Environ. Microbiol., 2009, 75, 5451.

142 A. J. Marsh, O. O'Sullivan, R. P. Ross, P. D. Cotter and C. Hill, BMC Genomics, 2010, 11, 679.

143 Q. Zhang, Y. Yu, J. E. Velasquez and W. A. van der Donk, Proc. Natl. Acad. Sci. U. S. A., 2012, DOI: 10.1073/ pnas.1210393109.

144 G. H. Völler, J. M. Krawczyk, A. Pesic, B. Krawczyk, J. Nachtigall and R. D. Süssmuth, ChemBioChem, 2012, 13, 1174.

145 T. Kupke, C. Kempter, V. Gnau, G. Jung and F. Götz, J. Biol. Chem., 1994, 269, 5653.

146 C. Heidrich, U. Pag, M. Josten, J. Metzger, R. W. Jack, G. Bierbaum, G. Jung and H. G. Sahl, Appl. Environ. Microbiol., 1998, 64, 3140.

147 D. A. Widdick, H. M. Dodd, P. Barraille, J. White, T. H. Stein, K. F. Chater, M. J. Gasson and M. J. Bibb, Proc. Natl. Acad. Sci. U. S. A., 2003, 100, 4316.

148 P. D. Cotter, P. M. O'Connor, L. A. Draper, E. M. Lawton, L. H. Deegan, C. Hill and R. P. Ross, Proc. Natl. Acad. Sci. U. S. A., 2005, 102, 18584

149 C. Chatterjee, M. Paul, L. Xie and W. A. van der Donk, Chem. Rev., 2005, 105, 633.

150 Y. Minami, K. Yoshida, R. Azuma, A. Urakawa, T. Kawauchi, T. Otani, K. Komiyama and S. Omura, Tetrahedron Lett., 1994, 35, 8001.

151 C. Kempter, T. Kupke, D. Kaiser, J. W. Metzger and G. Jung, Angew. Chem., Int. Ed. Engl., 1996, 35, 2104.

152 J. Claesen and M. J. Bibb, Proc. Natl. Acad. Sci. U. S. A., 2010, 107, 16297.

153 J. Claesen and M. J. Bibb, J. Bacteriol., 2011, 193, 2510.

154 T. Hamada, T. Sugawara, S. Matsunaga and N. Fusetani, Tetrahedron Lett., 1994, 35, 719.

155 U. Hentschel, J. Hopke, M. Horn, A. B. Friedrich, M. Wagner, J. Hacker and B. S. Moore, Appl. Environ. Microbiol., 2002, 68, 4431.

156 T. Hamada, S. Matsunaga, G. Yano and N. Fusetani, J. Am. Chem. Soc., 2005, 127, 110. 
157 N. Kessler, H. Schuhmann, S. Morneweg, U. Linne and M. A. Marahiel, J. Biol. Chem., 2004, 279, 7413.

158 T. Hamada, S. Matsunaga, M. Fujiwara, K. Fujita, H. Hirota, R. Schmucki, P. Guntert and N. Fusetani, J. Am. Chem. Soc., 2010, 132, 12941.

159 M. Iwamoto, H. Shimizu, I. Muramatsu and S. Oiki, FEBS Lett., 2010, 584, 3995.

160 P. A. Frey, A. D. Hegeman and F. J. Ruzicka, Crit. Rev. Biochem. Mol. Biol., 2008, 43, 63.

161 A. Jilek and G. Kreil, Monatsh. Chem., 2008, 139, 1.

162 Q. Zhang, W. A. van der Donk and W. Liu, Acc. Chem. Res., 2012, 45, 555.

163 R. Kellner, G. Jung, M. Josten, C. Kaletta, K. D. Entian and H. G. Sahl, Angew. Chem., 1989, 101, 618.

164 Besredka, Ann. Inst. Pasteur, 1901, 15, 880.

165 J. H. Brown, Monograph No. 9 Rockefeller Institute for Medical Research, New York, 1919.

166 J. T. Weld, J. Exp. Med., 1934, 59, 83.

167 E. W. Todd, J. Pathol. Bacteriol., 1938, 47, 423.

168 V. Nizet, B. Beall, D. J. Bast, V. Datta, L. Kilburn, D. E. Low and J. C. De Azavedo, Infect. Immun., 2000, 68, 4245.

169 V. Datta, S. M. Myskowski, L. A. Kwinn, D. N. Chiem, N. Varki, R. G. Kansal, M. Kotb and V. Nizet, Mol. Microbiol., 2005, 56, 681.

170 E. M. Molloy, P. D. Cotter, C. Hill, D. A. Mitchell and R. P. Ross, Nat. Rev. Microbiol., 2011, 9, 670.

171 P. D. Cotter, L. A. Draper, E. M. Lawton, K. M. Daly, D. S. Groeger, P. G. Casey, R. P. Ross and C. Hill, PLoS Pathog., 2008, 4, e1000144.

172 D. J. Gonzalez, S. W. Lee, M. E. Hensler, A. L. Markley, S. Dahesh, D. A. Mitchell, N. Bandeira, V. Nizet, J. E. Dixon and P. C. Dorrestein, J. Biol. Chem., 2010, 285, 28220.

173 K. L. Dunbar, J. O. Melby and D. A. Mitchell, Nat. Chem. Biol., 2012, 8, 569.

174 J. C. Milne, R. S. Roy, A. C. Eliot, N. L. Kelleher, A. Wokhlu, B. Nickels and C. T. Walsh, Biochemistry, 1999, 38, 4768.

175 E. W. Triplett and T. M. Barta, Plant Physiol., 1987, 85, 335.

176 A. J. Scupham and E. W. Triplett, J. Appl. Microbiol., 2006, 100, 500.

177 Y. Igarashi, Y. Kan, K. Fujii, T. Fujita, K. Harada, H. Naoki, H. Tabata, H. Onaka and T. Furumai, J. Antibiot., 2001, 54, 1045.

178 H. Onaka, H. Tabata, Y. Igarashi, Y. Sato and T. Furumai, J. Antibiot., 2001, 54, 1036.

179 R. Scholz, K. J. Molohon, J. Nachtigall, J. Vater, A. L. Markley, R. D. Süssmuth, D. A. Mitchell and R. Borriss, J. Bacteriol., 2011, 193, 215.

180 B. Kalyon, S. E. Helaly, R. Scholz, J. Nachtigall, J. Vater, R. Borriss and R. D. Süssmuth, Org. Lett., 2011, 13, 2996.

181 K. J. Molohon, J. O. Melby, J. Lee, B. S. Evans, K. L. Dunbar, S. B. Bumpus, N. L. Kelleher and D. A. Mitchell, ACS Chem. Biol., 2011, 6, 1307.

182 J. C. Milne, R. S. Roy, A. C. Eliot, N. L. Kelleher, A. Wokhlu, B. Nickels and C. T. Walsh, Biochemistry, 1999, 38, 4768.

183 J. A. McIntosh and E. W. Schmidt, ChemBioChem, 2010, 11, 1413.
184 J. A. McIntosh, M. S. Donia and E. W. Schmidt, J. Am. Chem. Soc., 2010, 132, 4089.

185 M. S. Donia and E. W. Schmidt, in Comprehensive Natural Products II Chemistry and Biology, ed. B. S. Moore and P. Crews, Elsevier: Oxford, 2010, p. 539.

186 C. Ireland and P. J. Scheuer, J. Am. Chem. Soc., 1980, 102, 5688.

187 T. W. Hambley, C. J. Hawkins, M. F. Lavin, A. Van den Brenk and D. J. Watters, Tetrahedron, 1992, 48, 341.

188 M. R. Prinsep, R. E. Moore, I. A. Levine and G. M. L. Patterson, J. Nat. Prod., 1992, 55, 140.

189 P. F. Long, W. C. Dunlap, C. N. Battershill and M. Jaspars, ChemBioChem, 2005, 6, 1760.

190 S. Sudek, M. G. Haygood, D. T. Youssef and E. W. Schmidt, Appl. Environ. Microbiol., 2006, 72, 4382.

191 N. Leikoski, D. P. Fewer and K. Sivonen, Appl. Environ. Microbiol., 2009, 75, 853.

192 N. Leikoski, D. P. Fewer, J. Jokela, M. Wahlsten, L. Rouhiainen and K. Sivonen, Appl. Environ. Microbiol., 2010, 76, 701.

193 M. S. Donia, W. F. Fricke, F. Partensky, J. Cox, S. I. Elshahawi, J. R. White, A. M. Phillippy, M. C. Schatz, J. Piel, M. G. Haygood, J. Ravel and E. W. Schmidt, Proc. Natl. Acad. Sci. U. S. A., 2011, 108, E1423.

194 M. S. Donia, W. F. Fricke, J. Ravel and E. W. Schmidt, PLoS One, 2011, 6, e17897.

195 M. S. Donia, D. E. Ruffner, S. Cao and E. W. Schmidt, ChemBioChem, 2011, 12, 1230.

196 K. Sivonen, N. Leikoski, D. P. Fewer and J. Jokela, Appl. Microbiol. Biotechnol., 2010, 86, 1213.

197 L. Cascales and D. J. Craik, Org. Biomol. Chem., 2010, 8, 5035.

198 N. H. Tan and J. Zhou, Chem. Rev., 2006, 106, 840.

199 H. Luo, H. E. Hallen-Adams, J. S. Scott-Craig and J. D. Walton, Eukaryotic Cell, 2010, 9, 1891.

200 L. A. Martin-Visscher, M. J. van Belkum and J. C. Vederas, in Prokaryotic Antimicrobial Peptides: From Genes to Applications, ed. D. Drider and S. Rebuffat, Springer Science, 2011, p. 213.

201 X. H. Jian, H. X. Pan, T. T. Ning, Y. Y. Shi, Y. S. Chen, Y. Li, X. W. Zeng, J. Xu and G. L. Tang, ACS Chem. Biol., 2012, 646.

202 J. A. McIntosh, C. R. Robertson, V. Agarwal, S. K. Nair, G. W. Bulaj and E. W. Schmidt, J. Am. Chem. Soc., 2010, 132, 15499.

203 J. Koehnke, A. Bent, W. E. Houssen, D. Zollman, F. Morawitz, S. Shirran, J. Vendome, A. F. Nneoyiegbe, L. Trembleau, C. H. Botting, M. C. M. Smith, M. Jaspars and J. H. Naismith, Nat. Struct. Mol. Biol., 2012, 19, 767.

204 J. A. McIntosh, M. S. Donia, S. K. Nair and E. W. Schmidt, J. Am. Chem. Soc., 2011, 133, 13698.

205 B. F. Milne, P. F. Long, A. Starcevic, D. Hranueli and M. Jaspars, Org. Biomol. Chem., 2006, 4, 631.

206 M. D. Tianero, M. S. Donia, T. S. Young, P. G. Schultz and E. W. Schmidt, J. Am. Chem. Soc., 2012, 134, 418.

207 W. E. Houssen and M. Jaspars, ChemBioChem, 2010, 11, 1803. 
208 A. R. Carroll, J. C. Coll, D. J. Bourne, J. K. MacLeod, T. M. Zabriskie, C. M. Ireland and B. F. Bowden, Aust. J. Chem., 1996, 49, 659.

209 A. B. Williams and R. S. Jacobs, Cancer Lett., 1993, 71, 97. 210 S. G. Aller, J. Yu, A. Ward, Y. Weng, S. Chittaboina, R. Zhuo, P. M. Harrell, Y. T. Trinh, Q. Zhang, I. L. Urbatsch and G. Chang, Science, 2009, 323, 1718.

211 P. Wipf, S. Venkatraman, C. P. Miller and S. J. Geib, Angew. Chem., 1994, 106, 1554.

212 A. Bertram and G. Pattenden, Nat. Prod. Rep., 2007, 24, 18.

213 T. L. Su, Br. J. Exp. Pathol., 1948, 29, 473.

214 M. C. Bagley, J. W. Dale, E. A. Merritt and X. Xiong, Chem. Rev., 2005, 105, 685.

215 M. C. Carnio, A. Holtzel, M. Rudolf, T. Henle, G. Jung and S. Scherer, Appl. Environ. Microbiol., 2000, 66, 2378.

216 J. Shoji, H. Hinoo, Y. Wakisaka, K. Koizumi, M. Mayama, S. Matsura and K. Matsumoto, J. Antibiot., 1976, 29, 366.

217 F. Benazet, M. Cartier, J. Florent, C. Godard, G. Jung, J. Lunel, D. Mancy, C. Pascal, J. Renaut, P. Tarridec, J. Theilleux, R. Tissier, M. Dubost and L. Ninet, Experientia, 1980, 36, 414.

218 J. Vandeputte and J. D. Dutcher, Antibiot. Annu., 1955, 3, 560.

219 O. D. Hensens and G. Albers-Schonberg, Tetrahedron Lett., 1978, 39, 3649.

220 O. D. Hensens and G. Albers-Schonberg, J. Antibiot., 1983, 36, 814 .

221 B. Anderson, D. C. Hodgkin and M. A. Viswamitra, Nature, 1970, 225, 233.

222 M. S. Puar, T. M. Chan, V. Hegde, M. Patel, P. Bartner, K. J. Ng, B. N. Pramanik and R. D. MacFarlane, J. Antibiot., 1998, 51, 221.

223 B. W. Bycroft and M. S. Gowland, J. Chem. Soc., Chem. Commun., 1978, 256.

224 M. C. Bagley and E. A. Merritt, J. Antibiot., 2004, 57, 829.

225 C. Pascard, A. Ducruix, J. Lunel and T. Prange, J. Am. Chem. Soc., 1977, 99, 6418.

226 T. Prange, A. Ducruix and C. Pascard, Nature, 1977, 265.

227 M. V. Rodnina, A. Savelsbergh, N. B. Matassova, V. I. Katunin, Y. P. Semenkov and W. Wintermeyer, Proc. Natl. Acad. Sci. U. S. A., 1999, 96, 9586.

228 P. H. Anborgh and A. Parmeggiani, EMBO J., 1991, 10, 779.

229 A. Mikolajka, H. Liu, Y. Chen, A. L. Starosta, V. Marquez, M. Ivanova, B. S. Cooperman and D. N. Wilson, Chem. Biol., 2011, 18, 589.

230 A. L. Starosta, H. Qin, A. Mikolajka, G. Y. Leung, K. Schwinghammer, K. C. Nicolaou, D. Y. Chen, B. S. Cooperman and D. N. Wilson, Chem. Biol., 2009, 16, 1087.

231 G. Lentzen, R. Klinck, N. Matassova, F. Aboul-ela and A. I. H. Murchie, Chem. Biol., 2003, 10, 769.

232 B. T. Porse, E. Cundliffe and R. A. Garrett, J. Mol. Biol., 1999, 287, 33.

233 B. T. Porse, I. Leviev, A. S. Mankin and R. A. Garrett, J. Mol. Biol., 1998, 276, 391.

234 D. M. Cameron, J. Thompson, P. E. March and A. E. Dahlberg, J. Mol. Biol., 2002, 319, 27.
235 R. L. Gonzalez, Jr., S. Chu and J. D. Puglisi, RNA, 2007, 13, 2091.

236 J. Modolell, B. Cabrer, A. Parmeggiani and D. Vazquez, Proc. Natl. Acad. Sci. U. S. A., 1971, 68, 1796.

237 J. M. Harms, D. N. Wilson, F. Schluenzen, S. R. Connell, T. Stachelhaus, Z. Zaborowska, C. M. T. Spahn and P. Fucini, Mol. Cell, 2008, 30, 26.

238 S. E. Heffron and F. Jurnak, Biochemistry, 2000, 39, 37.

239 A. Parmeggiani, I. M. Krab, S. Okamura, R. C. Nielsen, J. Nyborg and P. Nissen, Biochemistry, 2006, 45, 6846.

240 G. A. McConkey, M. J. Rogers and T. F. McCutchan, J. Biol. Chem., 1997, 272, 2046.

241 M. J. Rogers, E. Cundliffe and T. F. McCutchan, Antimicrob. Agents Chemother., 1998, 42, 715.

242 M. N. Aminake, S. Schoof, L. Sologub, M. Leubner, M. Kirschner, H. D. Arndt and G. Pradel, Antimicrob. Agents Chemother., 2011, 55, 1338.

243 S. Schoof, G. Pradel, M. N. Aminake, B. Ellinger, S. Baumann, M. Potowski, Y. Najajreh, M. Kirschner and H. D. Arndt, Angew. Chem., Int. Ed., 2010, 49, 3317.

244 U. G. Bhat, M. Halasi and A. L. Gartel, PLoS One, 2009, 4, e6593.

245 B. Pandit and A. L. Gartel, PLoS One, 2011, 6, e17110.

246 N. S. Hegde, D. A. Sanders, R. Rodriguez and S. Balasubramanian, Nat. Chem., 2011, 3, 725.

247 M. Aoki, T. Ohtsuka, M. Yamada, Y. Ohba, H. Yoshizaki, H. Yasuno, T. Sano, J. Watanabe, K. Yokose and H. Seto, J. Antibiot., 1991, 44, 582.

248 M. Hashimoto, T. Murakami, K. Funahashi, T. Tokunaga, K. Nihei, T. Okuno, T. Kimura, H. Naoki and H. Himeno, Bioorg. Med. Chem., 2006, 14, 8259.

249 N. Mizuhara, M. Kuroda, A. Ogita, T. Tanaka, Y. Usuki and K. Fujita, Bioorg. Med. Chem., 2011, 19, 5300.

250 D. J. Holmes, J. L. Caso and C. J. Thompson, EMBO J., 1993, $12,3183$.

251 Y. Ding, Y. Yu, H. Pan, H. Guo, Y. Li and W. Liu, Mol. BioSyst., 2010, 6, 1180.

252 K. Engelhardt, K. F. Degnes and S. B. Zotchev, Appl. Environ. Microbiol., 2010, 76, 7093.

253 J. Wang, Y. Yu, K. Tang, W. Liu, X. He, X. Huang and Z. Deng, Appl. Environ. Microbiol., 2010, 76, 2335.

254 T. S. Young and C. T. Walsh, Proc. Natl. Acad. Sci. U. S. A., 2011, 108, 13053.

255 Y. Yu, L. Duan, Q. Zhang, R. Liao, Y. Ding, H. Pan, E. Wendt-Pienkowski, G. Tang, B. Shen and W. Liu, ACS Chem. Biol., 2009, 4, 855.

256 A. A. Bowers, C. T. Walsh and M. G. Acker, J. Am. Chem. Soc., 2010, 132, 12182.

257 Q. Zhang, Y. Li, D. Chen, Y. Yu, L. Duan, B. Shen and W. Liu, Nat. Chem. Biol., 2011, 7, 154.

258 J. M. Waisvisz, M. G. van der Hoeven, J. van Peppen and W. C. M. Zwennis, J. Am. Chem. Soc., 1957, 79, 4520.

259 H. Shimamura, H. Gouda, K. Nagai, T. Hirose, M. Ichioka, Y. Furuya, Y. Kobayashi, S. Hirono, T. Sunazuka and S. Omura, Angew. Chem., Int. Ed., 2009, 48, 914.

260 Y. Kobayashi, M. Ichioka, T. Hirose, K. Nagai, A. Matsumoto, H. Matsui, H. Hanaki, R. Masuma, 
Y. Takahashi, S. Omura and T. Sunazuka, Bioorg. Med. Chem. Lett., 2010, 20, 6116.

261 T. Otaka and A. Kaji, J. Biol. Chem., 1976, 251, 2299.

262 T. Otaka and A. Kaji, FEBS Lett., 1981, 123, 173.

263 T. Otaka and A. Kaji, FEBS Lett., 1983, 153, 53.

264 J. M. Waisvisz, M. G. van der Hoeven and B. te Nijnhius, $J$. Am. Chem. Soc., 1957, 79, 4524.

265 M. Kaneda, J. Antibiot., 2002, 55, 924.

266 M. Kaneda, J. Antibiot., 1992, 45, 792.

267 H.-G. Lerchen, G. Schiffer, H. Brötz-Österheld, A. MayerBartschmid, S. Eckermann, C. Freiberg, R. Endermann, J. Schuhmann, H. Meier, N. Svenstrup, S. Seip, M. Gehling and D. Häbich, Cyclic iminopeptide derivatives, WO 2006/103010, 2006.

268 F. Baquero and F. Moreno, FEMS Microbiol. Lett., 1984, 23, 117.

269 K. Severinov, E. Semenova, A. Kazakov, T. Kazakov and M. S. Gelfand, Mol. Microbiol., 2007, 189, 8772.

270 S. Rebuffat, in Prokaryotic antimicrobial peptides: from genes to applications, ed. D. Drider and S. Rebuffat, Springer, New York, 2011, p. 55.

271 A. Bayer, S. Freund and G. Jung, Angew. Chem., Int. Ed. Engl., 1993, 32, 1336.

272 J. I. Guijarro, J. E. Gonzalez-Pastor, F. Baleux, J. L. San Millan, M. A. Castilla, M. Rico, F. Moreno and M. Delepierre, J. Biol. Chem., 1995, 270, 23520.

273 S. Rebuffat, A. Blond, D. Destoumieux-Garzon, C. Goulard and J. Peduzzi, Curr. Protein Pept. Sci., 2004, 5, 383.

274 G. Bierbaum and A. Jansen, Chem. Biol., 2007, 14, 734.

275 K. J. Rosengren and D. J. Craik, Chem. Biol., 2009, 16, 1211.

276 X. Thomas, D. Destoumieux-Garzon, J. Peduzzi, C. Afonso, A. Blond, N. Birlirakis, C. Goulard, L. Dubost, R. Thai, J. C. Tabet and S. Rebuffat, J. Biol. Chem., 2004, 279, 28233.

277 G. Vassiliadis, D. Destoumieux-Garzon, C. Lombard, S. Rebuffat and J. Peduzzi, Antimicrob. Agents Chemother., 2010, 54, 288.

278 K. Severinov, E. Semenova and T. Kazakov, in Prokaryotic antimicrobial peptides: from genes to applications, ed. D. Drider and S. Rebuffat, Springer, New York, 2011, p. 289.

279 M. J. Bayro, J. Mukhopadhyay, G. V. Swapna, J. Y. Huang, L. C. Ma, E. Sineva, P. E. Dawson, G. T. Montelione and R. H. Ebright, J. Am. Chem. Soc., 2003, 125, 12382.

280 K. J. Rosengren, R. J. Clark, N. L. Daly, U. Goransson, A. Jones and D. J. Craik, J. Am. Chem. Soc., 2003, 125, 12464.

281 K. A. Wilson, M. Kalkum, J. Ottesen, J. Yuzenkova, B. T. Chait, R. Landick, T. Muir, K. Severinov and S. A. Darst, J. Am. Chem. Soc., 2003, 125, 12475.

282 G. H. M. Vondenhoff, S. Dubiley, K. Severinov, E. Lescrinier, J. Rozenski and A. Van Aerschot, Bioorg. Med. Chem., 2011, 19, 5462 .

283 L. S. Håvarstein, D. B. Diep and I. F. Nes, Mol. Microbiol., 1995, 16, 229.

284 S. Duquesne, D. Destoumieux-Garzon, S. Zirah, C. Goulard, J. Peduzzi and S. Rebuffat, Chem. Biol., 2007, 14, 793.

285 D. J. Clarke and D. J. Campopiano, Org. Biomol. Chem., 2007, 5, 2564.
286 J. L. San Millan, R. Kolter and F. Moreno, J. Bacteriol., 1985, 163, 1016.

287 N. Allali, H. Afif, M. Couturier and L. Van Melderen, J. Bacteriol., 2002, 184, 3224.

288 R. F. Roush, E. M. Nolan, F. Lohr and C. T. Walsh, J. Am. Chem. Soc., 2008, 130, 3603.

289 E. M. Nolan, M. A. Fischbach, A. Koglin and C. T. Walsh, J. Am. Chem. Soc., 2007, 129, 14336.

290 E. M. Nolan and C. T. Walsh, Biochemistry, 2008, 47, 9289.

291 G. Vassiliadis, J. Peduzzi, S. Zirah, X. Thomas, S. Rebuffat and D. Destoumieux-Garzon, Antimicrob. Agents Chemother., 2007, 51, 3546.

292 M. O. Maksimov, S. J. Pan and A. J. Link, Nat. Prod. Rep., 2012, 29, 996.

293 K. Yano, S. Toki, S. Nakanishi, K. Ochiai, K. Ando, M. Yoshida, Y. Matsuda and M. Yamasaki, Bioorg. Med. Chem., 1996, 4, 115.

294 P. F. Lin, H. Samanta, C. M. Bechtold, C. A. Deminie, A. K. Patick, M. Alam, K. Riccardi, R. E. Rose, R. J. White and R. J. Colonno, Antimicrob. Agents Chemother., 1996, 40, 133.

295 M. Tsunakawa, S. L. Hu, Y. Hoshino, D. J. Detlefson, S. E. Hill, T. Furumai, R. J. White, M. Nishio, K. Kawano, S. Yamamoto, Y. Fukagawa and T. Oki, J. Antibiot., 1995, 48, 433.

296 G. Helynck, C. Dubertret, J. F. Mayaux and J. Leboul, J. Antibiot., 1993, 46, 1756.

297 Y. Morishita, S. Chiba, E. Tsukuda, T. Tanaka, T. Ogawa, M. Yamasaki, M. Yoshida, I. Kawamoto and Y. Matsuda, J. Antibiot., 1994, 47, 269.

298 M. Yamasaki, K. Yano, M. Yoshida, Y. Matsuda and K. Yamaguchi, J. Antibiot., 1994, 47, 276.

299 K. Yano, M. Yamasaki, M. Yoshida, Y. Matsuda and K. Yamaguchi, J. Antibiot., 1995, 48, 1368.

300 T. Ogawa, K. Ochiai, T. Tanaka, E. Tsukuda, S. Chiba, K. Yano, M. Yamasaki, M. Yoshida and Y. Matsuda, J. Antibiot., 1995, 48, 1213.

301 K. I. Kimura, F. Kanou, H. Takahashi, Y. Esumi, M. Uramoto and M. Yoshihama, J. Antibiot., 1997, 50, 373.

302 Y. Esumi, Y. Suzuki, Y. Itoh, M. Uramoto, K. Kimura, M. Goto, M. Yoshihama and T. Ichikawa, J. Antibiot., 2002, 55, 296.

303 W. Weber, W. Fischli, E. Hochuli, E. Kupfer and E. K. Weibel, J. Antibiot., 1991, 44, 164.

304 D. F. Wyss, H. W. Lahm, M. Manneberg and A. M. Labhardt, J. Antibiot., 1991, 44, 172.

305 M. Iwatsuki, R. Uchida, Y. Takakusagi, A. Matsumoto, C.-L. Jiang, Y. Takahashi, M. Arai, S. Kobayashi, M. Matsumoto, J. Inokoshi, H. Tomoda and S. Omura, J. Antibiot., 2007, 60, 357.

306 R. A. Salomon and R. N. Farias, J. Bacteriol., 1992, 174, 7428. 307 M. A. Delgado, M. R. Rintoul, R. N. Farias and R. A. Salomon, J. Bacteriol., 2001, 183, 4543.

308 T. A. Knappe, U. Linne, S. Zirah, S. Rebuffat, X. Xie and M. A. Marahiel, J. Am. Chem. Soc., 2008, 130, 11446.

309 K. Kuznedelov, E. Semenova, T. A. Knappe, D. Mukhamedyarov, A. Srivastava, S. Chatterjee, 
R. H. Ebright, M. A. Marahiel and K. Severinov, J. Mol. Biol., 2011, 412, 842.

310 T. A. Knappe, U. Linne, X. Xie and M. A. Marahiel, FEBS Lett., 2010, 584, 785.

311 M. O. Maksimov, I. Pelczer and A. J. Link, Proc. Natl. Acad. Sci. U. S. A., 2012, 109, 15223.

312 S. J. Pan, J. Rajniak, W. L. Cheung and A. J. Link, ChemBioChem, 2012, 13, 367.

313 K.-P. Yan, Y. Li, S. Zirah, C. Goulard, T. Knappe, M. Marahiel and S. Rebuffat, ChemBioChem, 2012, 13, 1046.

314 S. J. Pan, J. Rajniak, M. O. Maksimov and A. J. Link, Chem. Commun., 2012, 48, 1880.

315 J. O. Solbiati, M. Ciaccio, R. N. Farías, J. E. González-Pastor, F. Moreno and R. A. Salomón, J. Bacteriol., 1999, 181, 2659.

316 J. Inokoshi, M. Matsuhama, M. Miyake, H. Ikeda and H. Tomoda, Appl. Microbiol. Biotechnol., 2012, 95, 451.

317 R. Ducasse, K. P. Yan, C. Goulard, A. Blond, Y. Li, E. Lescop, E. Guittet, S. Rebuffat and S. Zirah, ChemBioChem, 2012, 13, 371.

318 M. O. Ishitsuka, T. Kusumi, H. Kakisawa, K. Kaya and M. M. Watanabe, J. Am. Chem. Soc., 1990, 112, 8180.

319 A. R. Weiz, K. Ishida, K. Makower, N. Ziemert, C. Hertweck and E. Dittmann, Chem. Biol., 2011, 18, 1413.

320 K. Miyamoto, H. Tsujibo, Y. Hikita, K. Tanaka, S. Miyamoto, M. Hishimoto, C. Imada, K. Kamei, S. Hara and Y. Inamori, Biosci., Biotechnol., Biochem., 1998, 62, 2446.

321 M. Murakami, Q. Sun, K. Ishida, H. Matsuda, T. Okino and K. Yamaguchi, Phytochemistry, 1997, 45, 1197.

322 T. Okino, H. Matsuda, M. Murakami and K. Yamaguchi, Tetrahedron, 1995, 51, 10679.

323 B. Philmus, G. Christiansen, W. Y. Yoshida and T. K. Hemscheidt, ChemBioChem, 2008, 9, 3066.

324 H. J. Shin, M. Murakami, H. Matsuda and K. Yamaguchi, Tetrahedron, 1996, 52, 8159.

325 K. Kanaori, K. Kamei, M. Taniguchi, T. Koyama, T. Yasui, R. Takano, C. Imada, K. Tajima and S. Hara, Biochemistry, 2005, 44, 2462.

326 T. Rohrlack, K. Christoffersen, M. Kaebernick and B. A. Neilan, Appl. Environ. Microbiol., 2004, 70, 5047.

327 N. Ziemert, K. Ishida, A. Liaimer, C. Hertweck and E. Dittmann, Angew. Chem., Int. Ed., 2008, 47, 7756.

328 M. Y. Galperin and E. V. Koonin, Protein Sci., 1997, 6, 2639.

329 B. Philmus, J. P. Guerrette and T. K. Hemscheidt, ACS Chem. Biol., 2009, 4, 429.

330 D. M. Gardiner, P. Waring and B. J. Howlett, Microbiology, 2005, 151, 1021.

331 D. H. Scharf, N. Remme, A. Habel, P. Chankhamjon, K. Scherlach, T. Heinekamp, P. Hortschansky, A. A. Brakhage and C. Hertweck, J. Am. Chem. Soc., 2011, 133, 12322.

332 K. Kawulka, T. Sprules, R. T. McKay, P. Mercier, C. M. Diaper, P. Zuber and J. C. Vederas, J. Am. Chem. Soc., 2003, 125, 4726.

333 T. Huang, H. Geng, V. R. Miyyapuram, C. S. Sit, J. C. Vederas and M. M. Nakano, J. Bacteriol., 2009, 191, 5690.
334 M. C. Rea, C. S. Sit, E. Clayton, P. M. O'Connor, R. M. Whittal, J. Zheng, J. C. Vederas, R. P. Ross and C. Hill, Proc. Natl. Acad. Sci. U. S. A., 2010, 107, 9352.

335 C. S. Sit, M. J. van Belkum, R. T. McKay, R. W. Worobo and J. C. Vederas, Angew. Chem., Int. Ed., 2011, 50, 8718.

336 W. T. Liu, Y. L. Yang, Y. Xu, A. Lamsa, N. M. Haste, J. Y. Yang, J. Ng, D. Gonzalez, C. D. Ellermeier, P. D. Straight, P. A. Pevzner, J. Pogliano, V. Nizet, K. Pogliano and P. C. Dorrestein, Proc. Natl. Acad. Sci. U. S. A., 2010, 107, 16286.

337 M. C. Rea, R. P. Ross, P. D. Cotter and C. Hill, in Prokaryotic Antimicrobial Peptides: From Genes to Applications, ed. D. Drider and S. Rebuffat, Springer Science, 2011, pp. 29-53. 338 J. Wang, Y. Yu, K. Tang, W. Liu, X. He, X. Huang and Z. Deng, Appl. Environ. Microbiol., 2010, 76, 2335.

339 M. Hashimoto, T. Murakami, K. Funahashi, T. Tokunaga, K. Nihei, T. Okuno, T. Kimura, H. Naoki and H. Himeno, Bioorg. Med. Chem., 2006, 14, 8259.

340 M. Aoki, T. Ohtsuka, M. Yamada, Y. Ohba, H. Yoshizaki, H. Yasuno, T. Sano, J. Watanabe, K. Yokose and H. Seto, J. Antibiot., 1991, 44, 582.

341 K. E. Kawulka, T. Sprules, C. M. Diaper, R. M. Whittal, R. T. McKay, P. Mercier, P. Zuber and J. C. Vederas, Biochemistry, 2004, 43, 3385.

342 C. S. Sit, R. T. McKay, C. Hill, R. P. Ross and J. C. Vederas, J. Am. Chem. Soc., 2011, 133, 7680.

343 M. C. Rea, A. Dobson, O. O’Sullivan, F. Crispie, F. Fouhy, P. D. Cotter, F. Shanahan, B. Kiely, C. Hill and R. P. Ross, Proc. Natl. Acad. Sci. U. S. A., 2011, 108(Suppl 1), 4639.

344 S. Thennarasu, D. K. Lee, A. Poon, K. E. Kawulka, J. C. Vederas and A. Ramamoorthy, Chem. Phys. Lipids, 2005, 137, 38.

345 K. Yamamoto, J. Xu, K. E. Kawulka, J. C. Vederas and A. Ramamoorthy, J. Am. Chem. Soc., 2010, 132, 6929.

346 L. Silkin, S. Hamza, S. Kaufman, S. L. Cobb and J. C. Vederas, Bioorg. Med. Chem. Lett., 2007, 18, 3103.

347 L. A. Martin-Visscher, S. Yoganathan, C. S. Sit, C. T. Lohans and J. C. Vederas, FEMS Microbiol. Lett., 2011, 317, 152.

348 G. Zheng, L. Z. Yan, J. C. Vederas and P. Zuber, J. Bacteriol., 1999, 181, 7346.

349 L. Flühe, T. A. Knappe, M. J. Gattner, A. Schäfer, O. Burghaus, U. Linne and M. A. Marahiel, Nat. Chem. Biol., 2012, 8, 350.

350 H. Lee, J. J. Churey and R. W. Worobo, FEMS Microbiol. Lett., 2009, 299, 205.

351 M. Sánchez-Hidalgo, M. Montalbán-Lopez, R. Cebrián, E. Valdivia, M. Martínez-Bueno and M. Maqueda, Cell. Mol. Life Sci., 2011, 68, 2845.

352 M. L. Kalmokoff, T. D. Cyr, M. A. Hefford, M. F. Whitford and R. M. Teather, Can. J. Microbiol., 2003, 49, 763.

353 Y. Kawai, T. Saito, H. Kitazawa and T. Itoh, Biosci., Biotechnol., Biochem., 1998, 62, 2438.

354 K. Arakawa, Y. Kawai, Y. Ito, K. Nakamura, T. Chujo, J. Nishimura, H. Kitazawa and T. Saito, Lett. Appl. Microbiol., 2010, 50, 406.

355 R. Kemperman, M. Jonker, A. Nauta, O. P. Kuipers and J. Kok, Appl. Environ. Microbiol., 2003, 69, 5839. 
356 K. Babasaki, T. Takao, Y. Shimonishi and K. Kurahashi, J. Biochem., 1985, 98, 585.

357 R. E. Wirawan, K. M. Swanson, T. Kleffmann, R. W. Jack and J. R. Tagg, Microbiology, 2007, 153, 1619.

358 L. A. Martin-Visscher, M. J. van Belkum, S. GarneauTsodikova, R. M. Whittal, J. Zheng, L. M. McMullen and J. C. Vederas, Appl. Environ. Microbiol., 2008, 74, 4756.

359 L. A. Martin-Visscher, X. Gong, M. Duszyk and J. C. Vederas, J. Biol. Chem., 2009, 284, 28674.

360 N. Sawa, T. Zendo, J. Kiyofuji, K. Fujita, K. Himeno, J. Nakayama and K. Sonomoto, Appl. Environ. Microbiol., 2009, 75, 1552.

361 Y. Masuda, H. Ono, H. Kitagawa, H. Ito, F. Mu, N. Sawa, T. Zendo and K. Sonomoto, Appl. Environ. Microbiol., 2011, 77, 8164.

362 J. Borrero, D. A. Brede, M. Skaugen, D. B. Diep, C. Herranz, I. F. Nes, L. M. Cintas and P. E. Hernandez, Appl. Environ. Microbiol., 2011, 77, 369.

363 M. J. Sánchez-Barrena, M. Martínez-Ripoll, A. Gálvez, E. Valdivia, M. Maqueda, V. Cruz and A. Albert, J. Mol. Biol., 2003, 334, 541.

364 X. Gong, L. A. Martin-Visscher, D. Nahirney, J. C. Vederas and M. Duszyk, Biochim. Biophys. Acta, Biomembr., 2009, 1788, 1797.

365 R. Cebrián, M. Maqueda, J. L. Neira, E. Valdivia, M. Martínez-Bueno and M. Montalbán-López, Appl. Environ. Microbiol., 2010, 76, 7268.

366 T. Wieland and H. Faulstich, Crit. Rev. Biochem. Mol. Biol., 1978, 5, 185.

367 D. A. Bushnell, P. Cramer and R. D. Kornberg, Proc. Natl. Acad. Sci. U. S. A., 2002, 99, 1218.

368 T. Wieland and V. M. Govindan, FEBS Lett., 1974, 46, 351.

369 H. Luo, H. E. Hallen-Adams and J. D. Walton, J. Biol. Chem., 2009, 284, 18070.

370 H. Luo, H. E. Hallen-Adams, J. S. Scott-Craig and J. D. Walton, Fungal Genet. Biol., 2012, 49, 123.

371 D. J. Craik, N. L. Daly, T. Bond and C. Waine, J. Mol. Biol., 1999, 294, 1327.

372 O. Saether, D. J. Craik, I. D. Campbell, K. Sletten, J. Juul and D. G. Norman, Biochemistry, 1995, 34, 4147.

373 L. Chiche, A. Heitz, J. C. Gelly, J. Gracy, P. T. Chau, P. T. Ha, J. F. Hernandez and D. Le-Nguyen, Curr. Protein Pept. Sci., 2004, 5, 341.

374 C. K. Wang, Q. Kaas, L. Chiche and D. J. Craik, Nucleic Acids Res., 2008, 36, D206.

375 U. Göransson and D. J. Craik, J. Biol. Chem., 2003, 278, 48188.

376 C. W. Gruber, A. G. Elliott, D. C. Ireland, P. G. Delprete, S. Dessein, U. Goransson, M. Trabi, C. K. Wang, A. B. Kinghorn, E. Robbrecht and D. J. Craik, Plant Cell, 2008, 20, 2471.

377 G. K. Nguyen, S. Zhang, N. T. Nguyen, P. Q. Nguyen, M. S. Chiu, A. Hardjojo and J. P. Tam, J. Biol. Chem., 2011, 286, 24275.

378 A. G. Poth, M. L. Colgrave, R. E. Lyons, N. L. Daly and D. J. Craik, Proc. Natl. Acad. Sci. U. S. A., 2011, 108, 10127.
379 N. L. Daly, K. J. Rosengren and D. J. Craik, Adv. Drug Delivery Rev., 2009, 61, 918.

380 B. L. Barbeta, A. T. Marshall, A. D. Gillon, D. J. Craik and M. A. Anderson, Proc. Natl. Acad. Sci. U. S. A., 2008, 105, 1221.

381 C. W. Gruber, M. Cemazar, M. A. Anderson and D. J. Craik, Toxicon, 2007, 49, 561.

382 C. Jennings, J. West, C. Waine, D. Craik and M. Anderson, Proc. Natl. Acad. Sci. U. S. A., 2001, 98, 10614.

383 C. V. Jennings, K. J. Rosengren, N. L. Daly, M. Plan, J. Stevens, M. J. Scanlon, C. Waine, D. G. Norman, M. A. Anderson and D. J. Craik, Biochemistry, 2005, 44, 851.

384 K. R. Gustafson, T. C. McKee and H. R. Bokesch, Curr. Protein Pept. Sci., 2004, 5, 331.

385 J. P. Tam, Y. A. Lu, J. L. Yang and K. W. Chiu, Proc. Natl. Acad. Sci. U. S. A., 1999, 96, 8913.

386 M. Pränting, C. Lööv, R. Burman, U. Göransson and D. I. Andersson, J. Antimicrob. Chemother., 2010, 65, 1964.

387 E. Svangard, U. Göransson, Z. Hocaoglu, J. Gullbo, R. Larsson, P. Claeson and L. Bohlin, J. Nat. Prod., 2004, 67, 144.

388 M. R. Plan, I. Saska, A. G. Cagauan and D. J. Craik, J. Agric. Food Chem., 2008, 56, 5237.

389 U. Göransson, M. Sjogren, E. Svangard, P. Claeson and L. Bohlin, J. Nat. Prod., 2004, 67, 1287.

390 M. L. Colgrave, A. C. Kotze, Y. H. Huang, J. O’Grady, S. M. Simonsen and D. J. Craik, Biochemistry, 2008, 47, 5581.

391 M. L. Colgrave, A. C. Kotze, D. C. Ireland, C. K. Wang and D. J. Craik, ChemBioChem, 2008, 9, 1939.

392 D. G. Barry, N. L. Daly, R. J. Clark, L. Sando and D. J. Craik, Biochemistry, 2003, 42, 6688.

393 D. J. Craik, M. Cemazar, C. K. Wang and N. L. Daly, Biopolymers, 2006, 84, 250.

394 K. Jagadish and J. A. Camarero, Biopolymers, 2010, 94, 611. 395 Y. H. Huang, M. L. Colgrave, N. L. Daly, A. Keleshian, B. Martinac and D. J. Craik, J. Biol. Chem., 2009, 284, 20699.

396 H. Kamimori, K. Hall, D. J. Craik and M. I. Aguilar, Anal. Biochem., 2005, 337, 149.

397 Z. O. Shenkarev, K. D. Nadezhdin, E. N. Lyukmanova, V. A. Sobol, L. Skjeldal and A. S. Arseniev, J. Inorg. Biochem., 2008, 102, 1246.

398 Z. O. Shenkarev, K. D. Nadezhdin, V. A. Sobol, A. G. Sobol, L. Skjeldal and A. S. Arseniev, FEBS J., 2006, 273, 2658.

399 C. W. Gruber, M. Cemazar, R. J. Clark, T. Horibe, R. F. Renda, M. A. Anderson and D. J. Craik, J. Biol. Chem., 2007, 282, 20435.

400 B. F. Conlan, A. D. Gillon, B. L. Barbeta and M. A. Anderson, Am. J. Bot., 2011, 98, 2018.

401 A. D. Gillon, I. Saska, C. V. Jennings, R. F. Guarino, D. J. Craik and M. A. Anderson, Plant J., 2008, 53, 505.

402 I. Saska, A. D. Gillon, N. Hatsugai, R. G. Dietzgen, I. HaraNishimura, M. A. Anderson and D. J. Craik, J. Biol. Chem., 2007, 282, 29721.

403 A. M. Broussalis, U. Göransson, J. D. Coussio, G. Ferraro, V. Martino and P. Claeson, Phytochemistry, 2001, 58, 47. 
404 B. Chen, M. L. Colgrave, C. Wang and D. J. Craik, J. Nat. Prod., 2006, 69, 23.

405 H. P. Kaufmann and A. Tobschirbel, Chem. Ber., 1959, 92, 2805.

406 H. Morita and K. Takeya, Heterocycles, 2010, 80, 739.

407 H. Morita, A. Shishido, T. Kayashita, M. Shimomura, K. Takeya and H. Itokawa, Chem. Lett., 1994, 12, 2415.

408 H. Morita, T. Kayashita, A. Uchida, K. Takeya and H. Itokawa, J. Nat. Prod., 1997, 60, 212.

409 B. Picur, M. Lisowski and I. Z. Siemion, Lett. Pept. Sci., 1998, $5,183$.

410 Y. Zhou, M.-K. Wang, S.-L. Peng and L.-S. Ding, Acta Bot. Sin, 2001, 43, 431.

411 J. A. Condie, G. Nowak, D. W. Reed, J. J. Balsevich, M. J. Reaney, P. G. Arnison and P. S. Covello, Plant J., 2011, 67, 682.

412 Sweet Orange Genome, http://www.citrusgenomedb.org/ species/sinensis.

413 Haploid Clementine Genome, International Citrus Genome Consortium, 2011, http://int-citrusgenomics.org, http://www.phytozome.net/clementine.

414 Z. Wang, N. Hobson, L. Galindo, J. McDill, L. Yang, S. Hawkins, G. Neutelings, R. Datla, G. Lambert, D. W. Galbraith, C. Cullis, G. K. S. Wong, J. Wang and M. K. Deyholos, 2011, GenBank accession number AFSQ00000000.

415 P. Venglat, D. Xiang, S. Qiu, S. L. Stone, C. Tibiche, D. Cram, M. Alting-Mees, J. Nowak, S. Cloutier, M. Deyholos, F. Bekkaoui, A. Sharpe, E. Wang, G. Rowland, G. Selvaraj and R. Datla, BMC Plant Biol., 2011, 11, 74.

416 P. S. Covello, R. S. S. Datla, S. L. Stone, J. J. Balsevich, M. J. T. Reaney, P. G. Arnison and J. A. Condie, WO/2010/ 130030, 2010.

417 S. Sato, H. Hirakawa, S. Isobe, E. Fukai, A. Watanabe, M. Kato, K. Kawashima, C. Minami, A. Muraki, N. Nakazaki, C. Takahashi, S. Nakayama, Y. Kishida, M. Kohara, M. Yamada, H. Tsuruoka, S. Sasamoto, S. Tabata, T. Aizu, A. Toyoda, T. Shin-i, Y. Minakuchi, Y. Kohara, A. Fujiyama, S. Tsuchimoto, S. Kajiyama, E. Makigano, N. Ohmido, N. Shibagaki, J. A. Cartagena, N. Wada, T. Kohinata, A. Atefeh, S. Yuasa, S. Matsunaga and K. Fukui, DNA Res., 2012, 18, 65.

418 A. J. J. van den Berg, S. F. A. Horsten, J. J. Kettenes-van den Bosch, B. H. Kroes, C. J. Beukelman, B. R. Leeflang and R. P. Labadie, FEBS Lett., 1995, 358, 215.

419 R. K. Devappa, H. P. Makkar and K. Becker, J. Agric. Food Chem., 2010, 58, 6543.

$420 \mathrm{http}: /$ www.ars-grin.gov/cgi-bin/npgs/html/taxfam.pl.

421 A. Arouri, V. Kiessling, L. Tamm, M. Dathe and A. Blume, J. Phys. Chem. B, 2011, 115, 158.

422 C. Auvin-Guette, C. Baraguey, A. Blond, F. Lezenven, J.-L. Pousset and B. Bodo, Tetrahedron Lett., 1997, 38, 2845.

423 H. Morita, A. Gonda, K. Takeya, H. Itokawa and Y. Iitaka, Tetrahedron, 1997, 53, 1617.

424 H. Morita, T. Kayashita, K. Takeya and H. Itokawa, Tetrahedron, 1994, 50, 12599.
425 H. Morita, Y. S. Yun, K. Takeya, H. Itokawa and K. Yamada, Tetrahedron, 1995, 51, 6003.

426 O. Buczek, G. Bulaj and B. M. Olivera, Cell. Mol. Life Sci., 2005, 62, 3067.

$427 \mathrm{H}$. Hu, P. Bandyopadhyay, B. M. Olivera and M. Yandell, BMC Genomics, 2011, 60.

428 Y. Terrat, D. Biass, S. Dutertre, P. Favreau, M. Remmd, R. Stocklin, D. Piquemal and F. Ducancel, Toxicon, 2012, 34.

429 B. M. Olivera, in Handbook of Biologically Active Peptides, ed. A. J. Kastin, Elsevier Inc, 2006, p. 381.

430 R. A. Seronay, A. E. Fedosov, M. A. Astilla, M. Watkins, N. Saguil, F. M. Heralde, S. Tagaro, G. T. Poppe, P. M. Alino, M. Oliverio, Y. I. Kantor, G. P. Concepcion and B. M. Olivera, Toxicon, 2010, 56, 1257.

431 B. M. Olivera, J. Imperial and G. P. Concepcion, in Handbook of Biologically Active Peptides, ed. A. J. Kastin, Elsevier, Inc., 2013.

432 M. Watkins, D. R. Hillyard and B. M. Olivera, J. Mol. Evol., 2006, 62, 247.

433 G. Bulaj and B. M. Olivera, Antioxid. Redox Signaling, 2008, $10,141$.

434 H. Terlau and B. M. Olivera, Physiol. Rev., 2004, 84, 41.

435 T. S. Han, R. W. Teichert, B. M. Olivera and G. Bulaj, Curr. Pharm. Des., 2008, 14, 2462.

436 V. D. Twede, G. Miljanich, B. M. Olivera and G. Bulaj, Curr. Opin. Drug Discov. Devel., 2009, 12, 231.

437 M. Prorok, S. E. Warder, T. Blandl and F. J. Castellino, Biochemistry, 1996, 35, 16528.

438 P. K. Bandyopadhyay, C. J. Colledge, C. S. Walker, L.-M. Zhou, D. R. Hillyard and B. M. Olivera, J. Biol. Chem., 1998, 273, 5447.

439 P. K. Bandyopadhyay, J. E. Garrett, R. P. Shetty, T. Keate, C. S. Walker and B. M. Olivera, Proc. Natl. Acad. Sci. U. S. A., 2002, 99, 1264.

440 T. J. Oman, J. M. Boettcher, H. Wang, X. N. Okalibe and W. A. van der Donk, Nat. Chem. Biol., 2011, 7, 78.

441 J. Stepper, S. Shastri, T. S. Loo, J. C. Preston, P. Novak, P. Man, C. H. Moore, V. Havlicek, M. L. Patchett and G. E. Norris, FEBS Lett., 2011, 585, 645.

442 H. Venugopal, P. J. Edwards, M. Schwalbe, J. K. Claridge, D. S. Libich, J. Stepper, T. Loo, M. L. Patchett, G. E. Norris and S. M. Pascal, Biochemistry, 2011, 50, 2748. 443 H. Wang and W. A. van der Donk, J. Am. Chem. Soc., 2011, 133, 16394.

444 G. Ji, R. Beavis and R. P. Novick, Science, 1997, 276, 2027. 445 M. Otto, R. Süssmuth, G. Jung and F. Götz, FEBS Lett., 1998, 424, 89.

446 A. Wuster and M. M. Babu, J. Bacteriol., 2008, 190, 743.

447 M. Thoendel, J. S. Kavanaugh, C. E. Flack and A. R. Horswill, Chem. Rev., 2011, 111, 117.

448 G. Ji, R. C. Beavis and R. P. Novick, Proc. Natl. Acad. Sci. U. S. A., 1995, 92, 12055.

449 M. Thoendel and A. R. Horswill, J. Biol. Chem., 2009, 284, 21828.

450 L. Zhang, L. Gray, R. P. Novick and G. Ji, J. Biol. Chem., 2002, $277,34736$. 
451 R. Qiu, W. Pei, L. Zhang, J. Lin and G. Ji, J. Biol. Chem., 2005, 280, 16695.

452 L. Zhang, J. Lin and G. Ji, J. Biol. Chem., 2004, 279, 19448.

453 M. Okada, I. Sato, S. J. Cho, H. Iwata, T. Nishio, D. Dubnau and Y. Sakagami, Nat. Chem. Biol., 2005, 1, 23.

454 M. Okada, Biosci., Biotechnol., Biochem., 2011, 75, 1413.

455 G. E. Kenney and A. C. Rosenzweig, ACS Chem. Biol., 2011, 7, 260.

456 H. J. Kim, D. W. Graham, A. A. DiSpirito, M. A. Alterman, N. Galeva, C. K. Larive, D. Asunskis and P. M. Sherwood, Science, 2004, 305, 1612.

457 L. A. Behling, S. C. Hartsel, D. E. Lewis, A. A. DiSpirito, D. W. Choi, L. R. Masterson, G. Veglia and W. H. Gallagher, J. Am. Chem. Soc., 2008, 130, 12604.

458 A. El Ghazouani, A. Basle, S. J. Firbank, C. W. Knapp, J. Gray, D. W. Graham and C. Dennison, Inorg. Chem., 2011, 50, 1378.

459 B. D. Krentz, H. J. Mulheron, J. D. Semrau, A. A. Dispirito, N. L. Bandow, D. H. Haft, S. Vuilleumier, J. C. Murrell, M. T. McEllistrem, S. C. Hartsel and W. H. Gallagher, Biochemistry, 2010, 49, 10117.

460 G. A. Somerville, A. Cockayne, M. Durr, A. Peschel, M. Otto and J. M. Musser, J. Bacteriol., 2003, 185, 6686.

461 E. Schiffmann, B. A. Corcoran and S. M. Wahl, Proc. Natl. Acad. Sci. U. S. A., 1975, 72, 1059.

462 Z. He, C. Yuan, L. Zhang and A. E. Yousef, FEBS Lett., 2008, $582,2787$.

463 Q. Zhang and W. A. van der Donk, FEBS Lett., 2012, 586, 3391.

464 M. Skaugen, J. Nissenmeyer, G. Jung, S. Stevanovic, K. Sletten, C. I. M. Abildgaard and I. F. Nes, J. Biol. Chem., 1994, 269, 27183.

465 C. Anthony, Biochem Soc Trans, 1998, 26, 413.

466 P. Mazodier, F. Biville, E. Turlin and F. Gasser, J. Gen. Microbiol., 1988, 134, 2513.

467 N. Goosen, H. P. Horsman, R. G. Huinen and P. van de Putte, J. Bacteriol., 1989, 171, 447.

468 N. Goosen, R. G. Huinen and P. van de Putte, J. Bacteriol., 1992, 174, 1426.

469 D. R. Houck, J. L. Hanners, C. J. Unkefer, M. A. van Kleef and J. A. Duine, Antonie van Leeuwenhoek, 1989, 56, 93.
470 J. J. Meulenberg, E. Sellink, N. H. Riegman and P. W. Postma, Mol. Gen. Genet., 1992, 232, 284.

471 J. S. Velterop, E. Sellink, J. J. Meulenberg, S. David, I. Bulder and P. W. Postma, J. Bacteriol., 1995, 177, 5088.

472 O. T. Magnusson, H. Toyama, M. Saeki, A. Rojas, J. C. Reed, R. C. Liddington, J. P. Klinman and R. Schwarzenbacher, Proc. Natl. Acad. Sci. U. S. A., 2004, 101, 7913.

473 S. R. Wecksler, S. Stoll, H. Tran, O. T. Magnusson, S. P. Wu, D. King, R. D. Britt and J. P. Klinman, Biochemistry, 2009, 48, 10151.

474 S. R. Wecksler, S. Stoll, A. T. Iavarone, E. M. Imsand, H. Tran, R. D. Britt and J. P. Klinman, Chem. Commun., 2010, 46, 7031.

475 Y. Oba, S. Kato, M. Ojika and S. Inouye, Biochem. Biophys. Res. Commun., 2009, 390, 684.

476 W. W. Ward and M. Chalfie, Expression of a gene for a modified green-fluorescent protein, US 5741 668, 1998.

477 M. Jin, L. Liu, S. A. Wright, S. V. Beer and J. Clardy, Angew. Chem., Int. Ed., 2003, 42, 2898.

478 M. Jin, S. A. Wright, S. V. Beer and J. Clardy, Angew. Chem., Int. Ed., 2003, 42, 2902.

479 S. E. Rokita, J. M. Adler, P. M. McTamney and J. A. Watson, Jr, Biochimie, 2010, 92, 1227.

480 N. Ziemert, K. Ishida, P. Quillardet, C. Bouchier, C. Hertweck, N. T. de Marsac and E. Dittmann, Appl. Environ. Microbiol., 2008, 74, 1791.

481 N. Garg, W. Tang, Y. Goto and W. A. van der Donk, Proc. Natl. Acad. Sci. U. S. A., 2012, 109, 5241.

482 S. R. Eddy, PLoS Comput. Biol., 2011, 7, e1002195.

483 J. D. Selengut, D. H. Haft, T. Davidsen, A. Ganapathy, M. Gwinn-Giglio, W. C. Nelson, A. R. Richter and O. White, Nucleic Acids Res., 2007, 35, D260.

484 M. Punta, P. C. Coggill, R. Y. Eberhardt, J. Mistry, J. Tate, C. Boursnell, N. Pang, K. Forslund, G. Ceric, J. Clements, A. Heger, L. Holm, E. L. Sonnhammer, S. R. Eddy, A. Bateman and R. D. Finn, Nucleic Acids Res., 2012, 40, D290.

485 A. de Jong, A. J. van Heel, J. Kok and O. P. Kuipers, Nucleic Acids Res., 2010, 38, W647.

486 J. S. Garavelli, Proteomics, 2004, 4, 1527.

487 D. H. Haft, Biol. Direct, 2009, 4, 15. 